\title{
Partial $L^{1}$ Monge-Kantorovich problem: variational formulation and numerical approximation
}

\author{
JOHN W. BARRETT \\ Department of Mathematics, Imperial College, London SW7 2AZ, UK \\ E-mail: j.barrett@imperial.ac.uk
}

LEONID PRIGOZHIN

Department of Solar Energy and Environmental Physics, Blaustein Institutes for Desert Research, Ben-Gurion University of the Negev, Sede Boqer Campus, 84990 Israel

E-mail: leonid@math.bgu.ac.il

[Received 30 September 2007 and in revised form 9 September 2008]

\begin{abstract}
We consider the Monge-Kantorovich problem with transportation cost equal to distance and a relaxed mass balance condition: instead of optimally transporting one given distribution of mass onto another with the same total mass, only a given amount of mass, $m$, has to be optimally transported. In this partial problem the given distributions are allowed to have different total masses and $m$ should not exceed the least of them. We derive and analyze a variational formulation of the arising free boundary problem in optimal transportation. Furthermore, we introduce and analyse the finite element approximation of this formulation using the lowest order Raviart-Thomas element. Finally, we present some numerical experiments where both approximations to the optimal transportation domains and the optimal transport between them are computed.
\end{abstract}

2000 Mathematics Subject Classification: 35D05, 35J85, 49J40, 65N12, 65N30, 82B27.

Keywords: Monge-Kantorovich problem, optimal transportation, free boundary, variational formulation, finite elements, augmented Lagrangian, convergence analysis.

\section{Introduction}

The classical Monge-Kantorovich (MK) problem in optimal transportation consists in finding an optimal mass preserving map from one distribution of mass, $f^{+}$, onto another one, $f^{-}$. In the relaxed formulation of this problem due to Kantorovich, the map is replaced by a transport plan, a measure minimizing the transportation cost

$$
\mathcal{C}:=\int_{\bar{\Omega} \times \bar{\Omega}} c(\underline{x}, \underline{y}) \gamma(\underline{x}, \underline{y})
$$

among all measures $\gamma \in \mathcal{M}^{+}(\bar{\Omega} \times \bar{\Omega})$ satisfying $\int_{A \times \bar{\Omega}} \gamma=\int_{A} f^{+}$and $\int_{\bar{\Omega} \times A} \gamma=\int_{A} f^{-}$for any Borel set $A \subseteq \bar{\Omega}$. Here $\Omega$ is a connected bounded open set in $\mathbb{R}^{n}$ such that the supports of $f^{+}$and $f^{-}$are in $\bar{\Omega}$; and $c(\underline{x}, \underline{y})$ is the cost function, usually determined by the distance $d_{\Omega}(\underline{x}, \underline{y})$ measured inside $\bar{\Omega}$. In addition, $\overline{\mathcal{M}}^{+}(\cdot)$ is the set of nonnegative Radon measures, whilst the set of all Radon measures will be denoted by $\mathcal{M}(\cdot)$. Clearly, if a solution exists, the two distributions should have the same mass, $m=\int_{\bar{\Omega}} f^{+}=\int_{\bar{\Omega}} f^{-}<\infty$.

The problem has a variety of applications and has been studied recently with a renewed interest (see [1, 13, 14, 21]). Numerical methods have been derived for the quadratic cost function (cost 
equals square of the distance; see [7, 3] and the references therein); and, more recently, for the so called $L^{1}$ MK problems where the cost function is linear (cost equals distance; see [5]).

In the latter work the $\operatorname{cost} c(\underline{x}, y)$ of transporting one unit of mass from a point $\underline{x} \in \bar{\Omega}$ to a point $\underline{y} \in \bar{\Omega}$ was assumed equal to the generalized distance

$$
d_{\Omega, k}(\underline{x}, \underline{y})=\inf \left\{\int_{0}^{1} k(s(t))\left|s^{\prime}(t)\right| \mathrm{d} t: s \in C^{0,1}([0,1] ; \bar{\Omega}), s(0)=\underline{x}, s(1)=\underline{y}\right\},
$$

where $k: \bar{\Omega} \rightarrow \mathbb{R}_{>0}$ is a given function. In this case an equivalent dual formulation of the MK problem (see [8] and also [1, 19]) can be written for a vectorial measure $q$, representing the transport flux,

$$
\mathcal{C}=\min \left\{\int_{\bar{\Omega}} k \underline{q} \mid: \underline{\nabla} \cdot \underline{q}=f^{+}-f^{-} \text {in } \Omega \text { and } \underline{q} \cdot \underline{v}=0 \text { on } \partial \Omega\right\} .
$$

Here $\partial \Omega$ is the boundary of $\Omega$ with normal $\underline{v}$; and the constraints are understood in the sense

$$
-\langle\underline{q}, \underline{\nabla} \varphi\rangle_{C(\bar{\Omega})}=\left\langle f^{+}-f^{-}, \varphi\right\rangle_{C(\bar{\Omega})} \quad \forall \varphi \in C^{1}(\bar{\Omega}),
$$

where $\langle\cdot, \cdot\rangle_{C(\bar{D})}$ is the duality pairing on $[C(\bar{D})]^{*} \times C(\bar{D})$ with $\mathcal{M}(\bar{D}) \equiv[C(\bar{D})]^{*}$ being the dual of $C(\bar{D})$, and is naturally extended to vector arguments. The dual formulation 1.3 was the basis of the present authors' numerical approximation in [5]. We note that the flux contains all the information on the direction and density of the optimal transportation and its cost; but there as well as in this paper, we do not find the optimal plan $\gamma$.

If the total masses do not agree, the maximal mass that can be transferred from $f^{+}$to $f^{-}$is $m=$ $\min \left\{\int_{\bar{\Omega}} f^{+}, \int_{\bar{\Omega}} f^{-}\right\}$. Determining an optimal transportation plan for this amount $m$ will be called an unbalanced MK problem. One can also seek an optimal transportation plan for a given amount of mass, $m<\min \left\{\int_{\bar{\Omega}} f^{+}, \int_{\bar{\Omega}} f^{-}\right\}$, which we will call a partial MK problem. The unbalanced and partial MK problems are free boundary problems, as the optimal transportation domains, as well as the optimal plan, have to be found. These problems have recently been introduced and studied theoretically, mainly for the quadratic cost function, by Caffarelli and McCann [10]. In this work we consider the unbalanced and partial MK problems with the linear transportation cost $\left(L^{1} \mathrm{MK}\right.$ problems).

First, we reformulate the problem as a balanced one over an extended region $\bar{\Omega}_{E} \supset \bar{\Omega}$ by introducing fictitious sources and an auxiliary zone of free transportation $\Sigma$ (a free Dirichlet region, see [9]) in such a way that if the balanced transportation plan is optimal, only the required mass $m$ is directly transported in $\bar{\Omega}$ from $f^{+}$to $f^{-}$, and this part of the transport plan is a solution to the partial MK problem.

Secondly, we make use of the dual formulation 1.3 , which is equivalent to the balanced $L^{1} \mathrm{MK}$ problem also if there is a closed free Dirichlet set. In this case $d_{\Omega_{E}, k}(x, y)$ becomes a semidistance, since $\left.k\right|_{\Sigma}=0$, and the equivalence was shown by Pratelli [19] under the assumption that $k \geqslant k_{0}>0$ in $\bar{\Omega}_{E} \backslash \Sigma$ and is a lower semicontinuous function. We will assume for simplicity that $k \equiv 1$ in $\bar{\Omega}$ and is extended to 1 in $\bar{\Omega}_{E} \backslash \Sigma$, and make use of the special structure of the auxiliary balanced problem to simplify the flux formulation 1.3 , and arrive at a new variational formulation of the partial $L^{1} \mathrm{MK}$ problem (see 2.7) below). A further simplification is possible for the unbalanced problem, where some constraints can be accounted for directly (see (2.10); and this is our reason to distinguish between these two cases. We note that a different approach to the resolution of unbalanced $L^{2} \mathrm{MK}$ problems has been suggested in [6]. 
Recently, Ekeland [11] has studied theoretically, for the quadratic cost function, the following optimal matching problem. Several kinds of goods, whose spatial distributions are given measures $f^{(i)} \in \mathcal{M}^{+}(\bar{\Omega})$ with the same total $\int_{\bar{\Omega}} f^{(i)}=m$, have to be brought together to produce $m$ units of a final product (one unit of each of these constitutive parts is necessary to manufacture one unit of the final product). It is required to determine the measure $g \in \mathcal{M}^{+}(\bar{\Omega})$ such that $\int_{\bar{\Omega}} g=m$ and the total cost of transporting all the measures $f^{(i)}$ onto $g$ is minimal. Here we show that our variational formulation of the unbalanced MK problem can be easily adapted (see (2.11) below), and used for the numerical approximation of the partial $L^{1}$ optimal matching problem for a given $m=\int_{\bar{\Omega}} g \leqslant \min \left\{\int_{\bar{\Omega}} f^{(i)}\right\}$ with the measures $f^{(i)}$ not necessarily balanced.

To approximate the problems numerically, we first regularize the nondifferentiable function $|\underline{v}|$ in $(2.7), 2.10)$ and $(2.11)$ by $(1 / r)|\underline{v}|^{r}$. In Section 3 , we prove existence and uniqueness for the Euler-Lagrange equations of these regularized problems; and, in addition, show subsequence convergence, as $r \rightarrow 1$, for the Euler-Lagrange inequality systems associated with 2.7), 22.10) and (2.11). This proves existence of a solution to these inequality systems and hence to the associated minimization problems (2.7), (2.10) and (2.11).

In Section 4 we discretize the resulting regularized Euler-Lagrange systems using the RaviartThomas finite element of the lowest order with vertex sampling on nonlinear terms. We prove wellposedness of these approximations; moreover, we prove convergence as the mesh parameter, $h$, goes to zero. In Section 5 we introduce, and prove convergence of, augmented Lagrangian methods to handle the constraints in the resulting problems. In Section 6 we discuss algorithms to solve the resulting nonlinear algebraic systems. Finally, in Section 7 we present numerical experiments to show the effectiveness of our approach. In these experiments, we use adaptive mesh refinement to improve the accuracy of the flux near its singularities and to enhance the resolution of the free boundaries surrounding the domains in $\bar{\Omega}$ from/to which the mass is directly transported.

Finally, we note that our formulation for the unbalanced MK problem collapses in the classical balanced case to an interesting modification of the standard variational formulation in terms of the transport flux. In addition, the derived numerical method for the unbalanced MK problem solves the balanced problem at least as efficiently as the mixed scheme in [5].

\section{Unbalanced and partial $L^{1}$ MK problems}

Let $\Omega \subset \mathbb{R}^{n}$ be a connected bounded open Lipschitz set, $f^{+}, f^{-} \in \mathcal{M}^{+}(\bar{\Omega})$ be the two nonnegative mass distributions and $m \in\left(0, \min \left\{\int_{\bar{\Omega}} f^{+}, \int_{\bar{\Omega}} f^{-}\right\}\right]$be the amount of mass that should be transported from $f^{+}$to $f^{-}$. Following [10] we define the set $\Gamma_{\leqslant}\left(f^{+}, f^{-}\right)$as the subset of $\mathcal{M}^{+}(\bar{\Omega} \times \bar{\Omega})$ whose left and right marginals are dominated by $f^{+}$and $f^{-}$, respectively, i.e. $\gamma \in \Gamma \leqslant\left(f^{+}, f^{-}\right)$if $\gamma \in \mathcal{M}^{+}(\bar{\Omega} \times \bar{\Omega})$ and

$$
\int_{A \times \bar{\Omega}} \gamma \leqslant \int_{A} f^{+} \text {and } \int_{\bar{\Omega} \times A} \gamma \leqslant \int_{A} f^{-}
$$

for all Borel sets $A \subseteq \bar{\Omega}$.

Our aim is to solve the partial (unbalanced) $L^{1} \mathrm{MK}$ problem, i.e. to minimize the cost functional

$$
\int_{\bar{\Omega} \times \bar{\Omega}} d_{\Omega, 1}(\underline{x}, \underline{y}) \gamma(\underline{x}, \underline{y})
$$

over all measures in $\Gamma_{\leqslant}^{m}\left(f^{+}, f^{-}\right):=\left\{\gamma \in \Gamma_{\leqslant}\left(f^{+}, f^{-}\right): \int_{\bar{\Omega} \times \bar{\Omega}} \gamma=m\right\}$. For ease of exposition, we have assumed here that $k \equiv 1$ in $\bar{\Omega}$. 


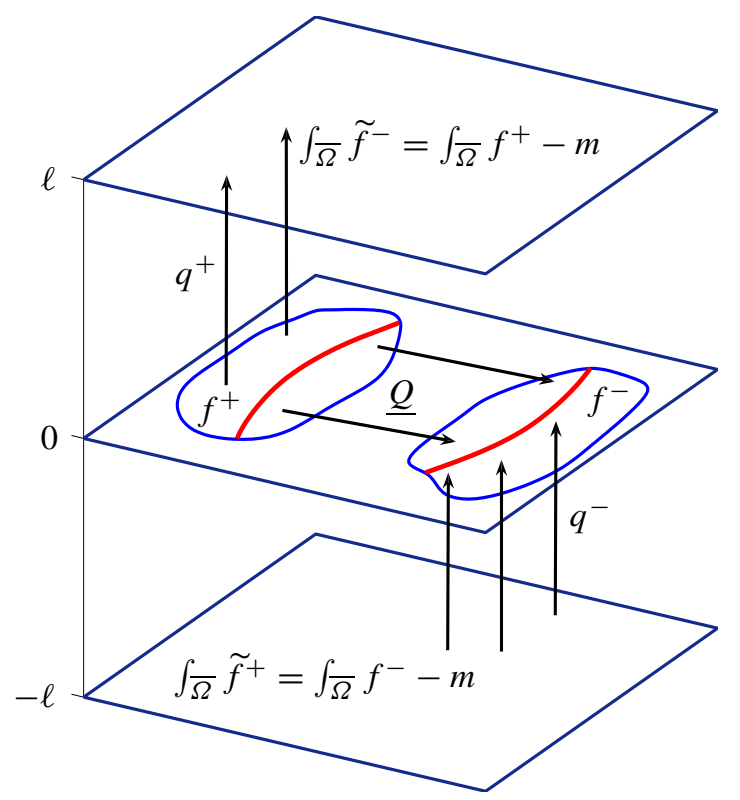

FIG. 1. The planes, sources and fluxes.

Let us imbed the space $\mathbb{R}^{n} \equiv\{\underline{x}\} \equiv\left\{x_{1}, \ldots, x_{n}\right\}$ into $\mathbb{R}^{n+1} \equiv\left\{\underline{x}, x_{n+1}\right\}$ as the subspace $x_{n+1}=0$. We then define two auxiliary sources, $\widetilde{f}^{-}, \widetilde{f}^{+} \in \mathcal{M}^{+}(\overline{\bar{\Omega}})$, supported in two parallel hyperplanes, $x_{n+1}=\ell$ and $x_{n+1}=-\ell$, respectively, where

$$
\ell>\frac{1}{2} \max \left\{d_{\Omega, 1}(\underline{x}, \underline{y}): \underline{x} \in \operatorname{supp}\left(f^{+}\right), \underline{y} \in \operatorname{supp}\left(f^{-}\right)\right\}
$$

and such that

$$
\int_{\bar{\Omega}} \tilde{f}^{+}=\int_{\bar{\Omega}} f^{-}-m \text { and } \int_{\bar{\Omega}} \tilde{f}^{-}=\int_{\bar{\Omega}} f^{+}-m ;
$$

see Figure 1. We assume that the transport in these two auxiliary hyperplanes, $x_{n+1}= \pm \ell$, is free, i.e. $k=0$ on them, so that the distribution of the sources in these hyperplanes is unimportant. We assume that $k=1$ everywhere else in $\mathbb{R}^{n+1}$. On noting 2.2 , the resulting MK problem is then balanced in $\bar{\Omega}_{E}:=\bar{\Omega} \times[-\ell, \ell]$ with

$$
\begin{aligned}
\int_{\bar{\Omega}_{E}}\left[f^{+}(\underline{x}) \delta\left(x_{n+1}\right)+\tilde{f}^{+}(\underline{x}) \delta\left(x_{n+1}+\ell\right)\right] & =\int_{\bar{\Omega}^{2}}\left[f^{+}+\tilde{f}^{+}\right]=\int_{\bar{\Omega}}\left[f^{+}+f^{-}\right]-m=\int_{\bar{\Omega}^{\prime}}\left[f^{-}+\tilde{f}^{-}\right] \\
& =\int_{\bar{\Omega}_{E}}\left[f^{-}(\underline{x}) \delta\left(x_{n+1}\right)+\tilde{f}^{-}(\underline{x}) \delta\left(x_{n+1}-\ell\right)\right],
\end{aligned}
$$

where $\delta \in \mathcal{M}(\mathbb{R})$ with $\int_{\mathbb{R}} \delta=1$ and $\operatorname{supp}(\delta)=\{0\}$. Since the auxiliary hyperplanes, $x_{n+1}= \pm \ell$, are at distance $\ell$ from the main one, $x_{n+1}=0$, it follows from (2.1) that the transportation cannot be optimal if any material is transported to the main hyperplane from an auxiliary one (or vice versa) and then back. Let $\Omega^{ \pm}$be open Lipschitz sets such that $\operatorname{supp}\left(f^{ \pm}\right) \subseteq \overline{\Omega^{ \pm}} \subseteq \bar{\Omega}$. Then as the transport in the auxiliary hyperplanes is free, optimal interplane transport brings, via straight 
transport rays orthogonal to the main hyperplane and all having the same length $\ell$, the mass $\int_{\bar{\Omega}} \widetilde{f}^{+}$ from the lower auxiliary hyperplane to $\overline{\Omega^{-}}$in the main hyperplane, and transfers the mass $\int_{\bar{\Omega}} \widetilde{f}^{-}$ from $\overline{\Omega^{+}}$in the main hyperplane to the upper hyperplane. We denote the corresponding fluxes as $q^{ \pm}(\underline{x}) \underline{e}_{n+1}, \underline{x} \in \overline{\Omega^{ \pm}}$, respectively, where $\underline{e}_{n+1} \in \mathbb{R}^{n+1}$ is the unit vector orthogonal to $\Omega$ and in the direction of increasing $x_{n+1}$. We also introduce the characteristic function $\chi_{A}$ such that $\chi_{A}(y)=1$ if $y \in A$, and otherwise $\chi_{A}(y)=0$. The optimal flux, $\underline{q} \in \mathbb{R}^{n+1}$, in $\bar{\Omega}_{E}$ should then have the following form:

$$
\begin{aligned}
\underline{q}\left(\underline{x}, x_{n+1}\right) & =[\underline{Q}(\underline{x})] \delta\left(x_{n+1}\right)+\left[q^{+}(\underline{x}) \chi_{(0, \ell)}\left(x_{n+1}\right)+q^{-}(\underline{x}) \chi_{(-\ell, 0)}\left(x_{n+1}\right)\right] \underline{e}_{n+1} \\
+ & {\left[\underline{q}_{F}^{+}(\underline{x})\right] \delta\left(x_{n+1}-\ell\right)+\left[\underline{q}_{F}^{-}(\underline{x})\right] \delta\left(x_{n+1}+\ell\right) \quad \forall \underline{x} \in \bar{\Omega}, x_{n+1} \in[-\ell, \ell] . }
\end{aligned}
$$

Here $\underline{Q}(\underline{x}) \in \mathbb{R}^{n}$ is the flux confined to the main hyperplane, $x_{n+1}=0$, and $\underline{q}_{F}^{ \pm}(\underline{x}) \in \mathbb{R}^{n}$ is the free transport flux in the auxiliary hyperplanes $x_{n+1}= \pm \ell$. In the above and below, we have extended $q^{ \pm}$from $\overline{\Omega^{ \pm}}$to $\bar{\Omega}$ by zero.

The equivalent dual flux formulation 1.3 of our auxiliary balanced MK problem with a free Dirichlet set on $\bar{\Omega}_{E}$ (see [19]) is

$$
\min \left\{\int_{\bar{\Omega}_{E}} k|\underline{q}|:-\langle\underline{q}, \underline{\nabla} \varphi\rangle_{C\left(\bar{\Omega}_{E}\right)}=\langle\tilde{f}, \varphi\rangle_{C\left(\bar{\Omega}_{E}\right)} \forall \varphi \in C^{1}\left(\bar{\Omega}_{E}\right)\right\}
$$

where $\tilde{f}=\left(f^{+}-f^{-}\right) \delta\left(x_{n+1}\right)+\tilde{f}^{+} \delta\left(x_{n+1}+\ell\right)-\tilde{f}^{-} \delta\left(x_{n+1}-\ell\right)$. Substituting 2.3 into this formulation we obtain

$$
\int_{\bar{\Omega}_{E}} k|\underline{q}|=\int_{\bar{\Omega}}\left[|\underline{Q}|+\ell\left(\left|q^{+}\right|+\left|q^{-}\right|\right)\right]
$$

Furthermore, the constraint on $\underline{q}$ over the domain $\bar{\Omega}_{E}$ reduces to three mass balance conditions over $\Omega$ :

$$
\begin{aligned}
-\langle\underline{Q}, \underline{\nabla} \varphi\rangle_{C(\bar{\Omega})}+\left\langle q^{+}-q^{-}, \varphi\right\rangle_{C(\bar{\Omega})} & =\left\langle f^{+}-f^{-}, \varphi\right\rangle_{C(\bar{\Omega})} & & \forall \varphi \in C^{1}(\bar{\Omega}), \\
\left\langle\underline{q}_{F}^{+}, \underline{\nabla} \varphi\right\rangle_{C(\bar{\Omega})}+\left\langle q^{+}, \varphi\right\rangle_{C(\bar{\Omega})} & =\left\langle\tilde{f}^{-}, \varphi\right\rangle_{C(\bar{\Omega})} & & \forall \varphi \in C^{1}(\bar{\Omega}), \\
-\left\langle\underline{q}_{F}^{-}, \underline{\nabla} \varphi\right\rangle_{C(\bar{\Omega})}+\left\langle q^{-}, \varphi\right\rangle_{C(\bar{\Omega})} & =\left\langle\tilde{f}^{+}, \varphi\right\rangle_{C(\bar{\Omega})} & & \forall \varphi \in C^{1}(\bar{\Omega}) .
\end{aligned}
$$

As we are not directly interested in the free flux $\underline{q}_{F}^{ \pm}$, since it does not appear in 2.4 , and the distribution of the auxiliary sources $\tilde{f}^{ \pm}$in $\bar{\Omega}$ is not important, we only need to consider constant test functions $\varphi$ in the equations $2.5 \mathrm{~b}, \mathrm{c})$. In which case, they collapse, on noting 2.2$]$, to the mass balance equations

$$
\int_{\bar{\Omega}} q^{+}=\int_{\bar{\Omega}} f^{+}-m \text { and } \int_{\bar{\Omega}} q^{-}=\int_{\bar{\Omega}} f^{-}-m .
$$

Defining $K_{\mathcal{P}} \subset[\mathcal{M}(\bar{\Omega})]^{n} \times \mathcal{M}\left(\overline{\Omega^{+}}\right) \times \mathcal{M}\left(\overline{\Omega^{-}}\right)$as the set of triples $\left(\underline{Q}, q^{+}, q^{-}\right)$satisfying 2.5a and 2.6, we arrive at the following variational formulation of the partial $L^{1}$ MK problem for given $\ell$ satisfying $[2.1]$ : 
(P) Minimize

$$
\int_{\bar{\Omega}}\left[|\underline{v}|+\ell\left(\left|v^{+}\right|+\left|v^{-}\right|\right)\right]
$$

over all $\left(\underline{v}, v^{+}, v^{-}\right) \in K_{\mathcal{P}}$.

We now show that a minimizer $\left(\underline{Q}, q^{+}, q^{-}\right) \in K_{\mathcal{P}}$ of $(\mathcal{P})$ is such that $q^{ \pm} \geqslant 0$; that is, under the assumption 2.1 , moving mass to/from an auxiliary plane and back cannot be optimal. If $q^{+} \ngtr 0$, then $q^{+}=g^{+}-g^{-}$, where $g^{ \pm} \in \mathcal{M}^{+}\left(\overline{\Omega^{+}}\right)$with $g^{-} \not \equiv 0$. It follows from $\sqrt{2.6}$ that $\int \frac{\Omega^{+}}{}\left(g^{+}-f^{+}\right)$

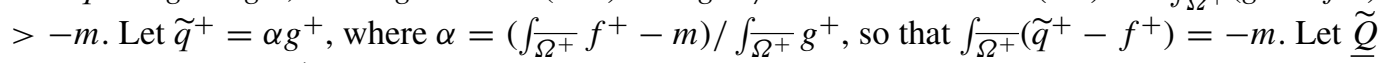
solve the balanced $L^{1} \mathrm{MK}$ problem

$$
\min \left\{\int_{\bar{\Omega}}|\underline{v}|:-\langle\underline{v}, \underline{\nabla} \varphi\rangle_{C(\bar{\Omega})}=\left\langle q^{+}-\tilde{q}^{+}, \varphi\right\rangle_{C(\bar{\Omega})} \forall \varphi \in C^{1}(\bar{\Omega})\right\} .
$$

It is easily deduced that $\left(\underline{Q}+\underline{\widetilde{Q}}, \widetilde{q}^{+}, q^{-}\right) \in K_{\mathcal{P}}$. Furthermore, as $\underline{\widetilde{Q}}$ optimally transfers the positive part of $q^{+}-\widetilde{q}^{+},\left[q^{+}-\widetilde{q}^{+}\right]_{+}=(1-\alpha) g^{+}$, to the negative part $g^{-}$, we have $\int \bar{\Omega} g^{-}=(1-\alpha) \int \bar{\Omega} g^{+}$ and

$$
\int_{\bar{\Omega}}\left|\widetilde{q}^{+}\right|+\ell \int_{\bar{\Omega}}\left|\widetilde{q}^{+}\right|<2 \ell(1-\alpha) \int_{\bar{\Omega}} g^{+}+\ell \alpha \int_{\bar{\Omega}} g^{+}=\ell \int_{\bar{\Omega}} g^{+}+\ell \int_{\bar{\Omega}} g^{-}=\ell \int_{\bar{\Omega}}\left|q^{+}\right| .
$$

It follows that

$$
\begin{aligned}
\int_{\bar{\Omega}}\left[|\underline{Q}+\underline{\underline{Q}}|+\ell\left(\left|\widetilde{q}^{+}\right|+\left|q^{-}\right|\right)\right] & \leqslant \int_{\bar{\Omega}}\left[|\underline{Q}|+|\underline{\widetilde{Q}}|+\ell\left(\left|\tilde{q}^{+}\right|+\left|q^{-}\right|\right)\right] \\
& <\int_{\bar{\Omega}}\left[|\underline{Q}|+\ell\left(\left|q^{+}\right|+\left|q^{-}\right|\right)\right]
\end{aligned}
$$

which contradicts $\left(Q, q^{+}, q^{-}\right) \in K_{\mathcal{P}}$ being a minimizer of $(\mathcal{P})$. Therefore $q^{+} \in \mathcal{M}^{+}\left(\overline{\Omega^{+}}\right)$. Similarly, we deduce that $q^{-} \in \mathcal{M}^{+}\left(\overline{\Omega^{-}}\right)$. Hence, the minimum value of 2.7 over $K_{\mathcal{P}}$ is $\int_{\bar{\Omega}}|\underline{Q}|+\ell\left[\int_{\bar{\Omega}}\left(f^{+}+f^{-}\right)-2 m\right]$, and it follows that a minimizer $\left(\underline{Q}, q^{+}, q^{-}\right) \in K_{\mathcal{P}}$ of 2.7$)$ is such that $\underline{Q}$ is the optimal flux for the original partial $L^{1} \mathrm{MK}$ problem.

In the unbalanced case the problem can be simplified. For example, if $m=\int_{\bar{\Omega}} f^{+} \leqslant \int_{\bar{\Omega}} f^{-}$, then it follows from the above that a minimizer $\left(Q, q^{+}, q^{-}\right)$of 2.7$]$ is such that $q^{+} \equiv 0$. Therefore 2.5a yields $q^{-}=\underline{\nabla} \cdot \underline{Q}-f^{+}+f^{-}$in $\Omega$ and $\underline{Q} \cdot \underline{v}=0$ on $\partial \Omega$ in a weak sense. Hence both conditions in 2.6) are satisfied automatically; similarly, if $m=\int_{\bar{\Omega}} f^{-} \leqslant \int_{\bar{\Omega}} f^{+}$. Setting

$$
\begin{aligned}
\underline{V}_{0}^{\mathcal{M}}(\Omega):=\left\{\underline{v} \in[\mathcal{M}(\bar{\Omega})]^{n}: \exists w:=\underline{\nabla} \cdot \underline{v} \in \mathcal{M}(\bar{\Omega})\right. \text { such that } \\
\\
\left.\langle w, \varphi\rangle_{C(\bar{\Omega})}=-\langle\underline{v}, \underline{\nabla} \varphi\rangle_{C(\bar{\Omega})} \forall \varphi \in C^{1}(\bar{\Omega})\right\},
\end{aligned}
$$

we have the following variational formulation of the unbalanced $L^{1}$ MK problem for given $\ell$ satisfying 2.1):

$(\mathcal{U})$ Minimize

$$
\int_{\bar{\Omega}}\left[|\underline{v}|+\ell\left|\underline{\nabla} \cdot \underline{v}-f^{+}+f^{-}\right|\right]
$$

over all $\underline{v} \in \underline{V}_{0}^{\mathcal{M}}(\Omega)$. 
For the balanced $L^{1} \mathrm{MK}$ problem, we deduce from 2.77 , as $q^{+} \equiv q^{-} \equiv 0$, the known variational formulation (1.3) with $k \equiv 1$. However, here we also obtain (2.10) as an alternative formulation, which is equivalent provided $\ell$ satisfies the inequality (2.1).

Let us now assume we are given measures $f^{(i)} \in \mathcal{M}^{+}(\bar{\Omega}), i=1 \rightarrow I$, and we want to transport the same amount of mass, $m \leqslant \min \left\{\int_{\bar{\Omega}} f^{(i)}\right\}$, from each of these distributions onto another measure, $g \in \mathcal{M}^{+}(\bar{\Omega})$ with $\int_{\bar{\Omega}} g=m$. We need then to solve $I$ unbalanced MK problems 2.10 with $f^{+}=$ $f^{(i)}, f^{-}=g$. Since these problems are independent, the optimal transportation fluxes $\underline{Q}^{(i)}$ can also be found, if $g$ was given, by minimizing over $\left\{\underline{v}^{(i)}\right\}_{i=1}^{I}$, where $\underline{v}^{(i)} \in \underline{V}_{0}^{\mathcal{M}}(\Omega)$, the functional $\sum_{i=1}^{I} \int_{\bar{\Omega}}\left[\left|\underline{v}^{(i)}\right|+\ell\left|\underline{\nabla} \cdot \underline{v}^{(i)}-f^{(i)}+g\right|\right]$ with $\ell$ sufficiently large; e.g. $\ell>\frac{1}{2} \operatorname{diam}(\Omega)$. The partial optimal matching problem consists in determining the measure $g \in \mathcal{M}^{+}(\bar{\Omega})$ for which the total transportation cost $\sum_{i=1}^{I} \int_{\bar{\Omega}}\left|\underline{v}^{(i)}\right|$ is minimal. This leads to the following variational formulation of the partial $L^{1}$ optimal matching problem for given $\ell>\frac{1}{2} \operatorname{diam}(\Omega)$ :

$(\mathcal{P M})$ Minimize

$$
\sum_{i=1}^{I} \int_{\bar{\Omega}}\left[\left|\underline{v}^{(i)}\right|+\ell\left|\underline{\nabla} \cdot \underline{v}^{(i)}-f^{(i)}+g\right|\right]
$$

over all $\left\{\underline{v}^{(i)}\right\}_{i=1}^{I}, \underline{v}^{(i)} \in \underline{V}_{0}^{\mathcal{M}}(\Omega)$, and $g \in \mathcal{M}^{+}(\bar{\Omega})$ satisfying $\int_{\bar{\Omega}} g=m$.

We note that in some applications one may want to restrict the support of $g$ to a given set $\bar{\Omega}_{g} \subset \bar{\Omega}$, and hence seek $g \in \mathcal{M}^{+}\left(\bar{\Omega}_{g}\right)$ satisfying $\int_{\bar{\Omega}_{g}} g=m$. In addition, one can simplify the problem by assuming that $g \in L^{\infty}\left(\Omega_{g}\right)$ with $g \leqslant G$ for a given constant $G \geqslant m /\left|\Omega_{g}\right|$, which we shall do in this paper.

We end this section with a few remarks about the notation employed in this paper. Throughout we adopt the standard notation for Sobolev spaces on a bounded Lipschitz open set $D$, denoting the norm of $W^{j, p}(D)(j \in \mathbb{N}, p \in[1, \infty])$ by $\|\cdot\|_{j, p, D}$ and the seminorm by $|\cdot|_{j, p, D}$. Of course, we have $|\cdot|_{0, p, D} \equiv\|\cdot\|_{0, p, D}$. We extend these norms and seminorms in the natural way to the corresponding spaces of vector-valued functions. For $p=2, W^{j, 2}(D)$ will be denoted by $H^{j}(D)$ with the associated norm and seminorm written as, respectively, $\|\cdot\|_{j, D}$ and $|\cdot|_{j, D}$. We introduce $L_{M}^{p}(D):=\left\{\eta \in L^{p}(D): \int_{D} \eta \mathrm{d} \underline{x}=0\right\}$, and recall the Poincaré inequality for any $p \in[1, \infty]:$

$$
|\eta|_{0, p, D} \leqslant C_{D}^{*}|\underline{\nabla} \eta|_{0, p, D} \quad \forall \eta \in W_{M}^{1, p}(D):=W^{1, p}(D) \cap L_{M}^{p}(D),
$$

where $C_{D}^{*}$ depends on $D$, but is independent of $p$. The measure of $D$ will be denoted by $|D|$. Throughout, for any Banach space $V$, its dual will be denoted by $V^{*}$.

For our regularized problems in the next section we require, for $r \in(1, \infty)$, the reflexive Banach space

$$
\underline{V}_{0}^{r}(\Omega):=\left\{\underline{v} \in \underline{V}^{r}(\Omega): \underline{v} \cdot \underline{v}=0 \text { on } \partial \Omega\right\}
$$

where

$$
\underline{V}^{r}(\Omega):=\left\{\underline{v} \in\left[L^{r}(\Omega)\right]^{n}: \underline{\nabla} \cdot \underline{v} \in L^{r}(\Omega)\right\},
$$

with norm

$$
\|\underline{v}\|_{\underline{\underline{V}}^{r}(\Omega)}:=\left[|\underline{v}|_{0, r, \Omega}^{r}+|\underline{\nabla} \cdot \underline{v}|_{0, r, \Omega}^{r}\right]^{1 / r} .
$$


We note that $\underline{V}_{0}^{r}(\Omega)$ is the strong closure of $\left[C_{0}^{\infty}(\Omega)\right]^{n}$ in the norm $\|\cdot\|_{\underline{V}^{r}(\Omega)}$; this can be shown e.g. by a simple extension of the argument for the case $r=2$ in [18, pp. 26-29].

Let $C(\bar{D})$ denote the space of continuous functions on $\bar{D}$, and $C_{M}(\bar{D}):=\left\{\eta \in C(\bar{D}): \int_{D} \eta\right.$ $=0\}$. As one can identify $L^{1}(D)$ with a closed subspace of $\mathcal{M}(\bar{D})$, it is convenient to adopt the notation

$$
\int_{\bar{D}}|\mu| \equiv\|\mu\|_{\mathcal{M}(\bar{D})}:=\sup _{\substack{\eta \in C(\bar{D}) \\|\eta|_{0, \infty, D} \leqslant 1}}\langle\mu, \eta\rangle_{C(\bar{D})}<\infty
$$

We note that if $\left\{\mu_{j}\right\}_{j} \geqslant 0$ is a bounded sequence in $\mathcal{M}(\bar{D})$, then there exist a subsequence $\left\{\mu_{j_{\ell}}\right\}_{j_{\ell}} \geqslant 0$ and a $\mu \in \mathcal{M}(\bar{D})$ such that as $j_{\ell} \rightarrow \infty$,

$$
\mu_{j_{\ell}} \rightarrow \mu \quad \text { vaguely in } \mathcal{M}(\bar{D}), \quad \text { i.e. } \quad\left\langle\mu_{j_{\ell}}-\mu, \eta\right\rangle_{C(\bar{D})} \rightarrow 0 \quad \forall \eta \in C(\bar{D}) .
$$

In addition, we have

$$
\liminf _{j_{\ell} \rightarrow \infty} \int_{\bar{D}}\left|\mu_{j_{\ell}}\right| \geqslant \int_{\bar{D}}|\mu|
$$

see e.g. [12, p. 5] and [17, p. 223]. Moreover, we note that for all $\underline{v} \in \underline{V}_{0}^{\mathcal{M}}(\Omega)$, there exists $\underline{v}_{j} \in$ $\left[C_{0}^{\infty}(\Omega)\right]^{n}$ such that

$$
\begin{aligned}
& \underline{v}_{j} \rightarrow \underline{v} \quad \text { vaguely in }[\mathcal{M}(\bar{\Omega})]^{n}, \quad \underline{\nabla} \cdot \underline{v}_{j} \rightarrow \underline{\nabla} \cdot \underline{v} \quad \text { vaguely in } \mathcal{M}(\bar{\Omega}) \quad \text { as } j \rightarrow \infty, \\
& \underset{j \rightarrow \infty}{\limsup } \int_{\bar{\Omega}}\left|\underline{v}_{j}\right| \leqslant \int_{\bar{\Omega}}|\underline{v}| ;
\end{aligned}
$$

e.g. see the proof of Lemma 2.4 in [5], which is based on the standard techniques of partition of unity, local change of variable and mollification. Similarly to $2.17 \mathrm{a}, \mathrm{b})$, for all $v \in \mathcal{M}(\bar{\Omega})$ there exists $v_{j} \in C_{0}^{\infty}(\Omega)$ such that

$$
v_{j} \rightarrow v \quad \text { vaguely in } \mathcal{M}(\bar{\Omega}) \quad \text { as } j \rightarrow \infty, \quad \text { and } \quad \limsup _{j \rightarrow \infty} \int_{\bar{\Omega}}\left|v_{j}\right| \leqslant \int_{\bar{\Omega}}|v| .
$$

Finally, throughout $C$ denotes a generic positive constant independent of the regularization parameter, $r \in(1, \infty)$, and the mesh parameter $h$, whereas $C_{s_{1}, s_{2}, \cdots s_{I}}$ denotes a generic positive constant dependent on $\left\{s_{i}\right\}_{i=1}^{I}$.

\section{Existence theory for $(\mathcal{P}),(\mathcal{U})$ and $(\mathcal{P} \mathcal{M})$ via regularization}

Firstly, we gather together our assumptions on the data.

(A1) Let $\Omega \subset \mathbb{R}^{n}, n \geqslant 1$, be a bounded connected open set with a Lipschitz boundary $\partial \Omega$, if $n \geqslant 2$. For $(\mathcal{P})$ we assume that $f^{ \pm} \in \mathcal{M}^{+}\left(\overline{\Omega^{ \pm}}\right)$with $\Omega^{ \pm}$an open Lipschitz subset of $\Omega$, and $m \in\left(0, \min \left\{\int \overline{\Omega^{ \pm}} f^{ \pm}\right\}\right]$. In all cases, for simplicity, we choose the parameter $\ell>\frac{1}{2} \operatorname{diam}(\Omega)$ with respect to $d_{\Omega, 1}$ (recall $(1.2)$ ).

Of course, one could assume the relaxed version 2.1 in the case of $(\mathcal{P})$ and $(\mathcal{U})$.

Throughout, we extend functions defined on subsets $D$ of $\Omega$ by zero to $\Omega$; and in addition, we define $\left(\eta_{1}, \eta_{2}\right)_{D}:=\int_{D} \eta_{1} \eta_{2} \mathrm{~d} \underline{x}$, and this is naturally extended to vector functions. 
For any $r>1$, we regularize the nondifferentiable nonlinearity $|\cdot|$ by the strictly convex function $(1 / r)|\cdot|^{r}$. We note that for all $\underline{b}, \underline{c} \in \mathbb{R}^{n}$,

$$
\frac{1}{r} \frac{\partial|\underline{b}|^{r}}{\partial b_{i}}=|\underline{b}|^{r-2} b_{i}, \quad \text { so } \quad|\underline{b}|^{r-2} \underline{b} \cdot(\underline{b}-\underline{c}) \geqslant \frac{1}{r}\left[|\underline{b}|^{r}-|\underline{c}|^{r}\right] .
$$

In addition, for $r \in(1,2]$ and all $\underline{b}, \underline{c} \in \mathbb{R}^{n}$ we have

$$
\begin{aligned}
\left(|\underline{b}|^{r-2} \underline{b}-|\underline{c}|^{r-2} \underline{c}\right) \cdot(\underline{b}-\underline{c}) & =\left(|\underline{b}|^{r-1}-|\underline{c}|^{r-1}\right)(|\underline{b}|-|\underline{c}|)+\left(|\underline{b}|^{r-2}+|\underline{c}|^{r-2}\right)(|\underline{b}||\underline{c}|-\underline{b} \cdot \underline{c}) \\
& \geqslant(r-1)(|\underline{b}|+|\underline{c}|)^{r-2}\left[(|\underline{b}|-|\underline{c}|)^{2}+2(|\underline{b}||\underline{c}|-\underline{b} \cdot \underline{c})\right] \\
& \geqslant(r-1)(|\underline{b}|+|\underline{c}|)^{r-2}|\underline{b}-\underline{c}|^{2} .
\end{aligned}
$$

\subsection{Existence for $(\mathcal{P})$}

For a given $r>1$, on setting $p=r /(r-1)$ so that $1 / r+1 / p=1$, we consider the following problem for given $f_{r}^{ \pm} \in L^{r}\left(\Omega_{r}^{ \pm}\right)$with $\overline{\Omega^{ \pm}} \subseteq \overline{\Omega_{r}^{ \pm}} \subseteq \bar{\Omega}$ and $\left(f_{r}^{ \pm}, 1\right)_{\Omega_{r}^{ \pm}}=\int_{\overline{\Omega^{ \pm}}} f^{ \pm}$:

$\left(\mathcal{P}_{r}\right)$ Find $\underline{Q}_{r} \in \underline{V}_{0}^{r}(\Omega), q_{r}^{ \pm} \in L^{r}\left(\Omega_{r}^{ \pm}\right), u_{r} \in L_{M}^{p}(\Omega)$ and $\lambda_{r}^{ \pm} \in \mathbb{R}$ such that

$$
\begin{aligned}
\left(\left|\underline{Q}_{r}\right|^{r-2} \underline{Q}_{r}, \underline{v}\right)_{\Omega} & =\left(u_{r}, \underline{\nabla} \cdot \underline{v}\right)_{\Omega} & & \forall \underline{v} \in \underline{V}_{0}^{r}(\Omega), \\
\ell\left(\left|q_{r}^{+}\right|^{r-2} q_{r}^{+}, v^{+}\right)_{\Omega_{r}^{+}} & =\left(u_{r}-\lambda_{r}^{+}, v^{+}\right)_{\Omega_{r}^{+}} & & \forall v^{+} \in L^{r}\left(\Omega_{r}^{+}\right), \\
\ell\left(\left|q_{r}^{-}\right|^{r-2} q_{r}^{-}, v^{-}\right)_{\Omega_{r}^{-}} & =-\left(u_{r}+\lambda_{r}^{-}, v^{-}\right)_{\Omega_{r}^{-}} & & \forall v^{-} \in L^{r}\left(\Omega_{r}^{-}\right), \\
\left(\underline{\nabla} \cdot \underline{Q}_{r}, \eta\right)_{\Omega} & =\left(f_{r}^{+}-q_{r}^{+}, \eta\right)_{\Omega_{r}^{+}}-\left(f_{r}^{-}-q_{r}^{-}, \eta\right)_{\Omega_{r}^{-}} & & \forall \eta \in L^{p}(\Omega), \\
\left(f_{r}^{+}-q_{r}^{+}, 1\right)_{\Omega_{r}^{+}} & =\left(f_{r}^{-}-q_{r}^{-}, 1\right)_{\Omega_{r}^{-}}=m . & &
\end{aligned}
$$

For $\rho \in L_{M}^{r}(\Omega)$, let

$$
\underline{X}^{r}(\rho):=\left\{\underline{v} \in \underline{V}_{0}^{r}(\Omega):(\underline{\nabla} \cdot \underline{v}, \eta)_{\Omega}=(\rho, \eta)_{\Omega} \forall \eta \in L^{p}(\Omega)\right\} .
$$

In addition, we introduce

$$
\begin{array}{r}
\underline{Y}^{r}\left(f_{r}^{+}, f_{r}^{-}, m\right):=\left\{\left(\underline{v}, v^{+}, v^{-}\right) \in \underline{X}^{r}\left(\left[f_{r}^{+}-v^{+}\right]-\left[f_{r}^{-}-v^{-}\right]\right) \times L^{r}\left(\Omega_{r}^{+}\right) \times L^{r}\left(\Omega_{r}^{-}\right):\right. \\
\left.\left(f_{r}^{+}-v^{+}, 1\right)_{\Omega_{r}^{+}}=\left(f_{r}^{-}-v^{-}, 1\right)_{\Omega_{r}^{-}}=m\right\} .
\end{array}
$$

It follows from 3.1, 3.4 and 3.5 that a solution of $\left(\mathcal{P}_{r}\right)$ is such that $\left(\underline{Q}_{r}, q_{r}^{+}, q_{r}^{-}\right) \in$ $\underline{Y}^{r}\left(f_{r}^{+}, f_{r}^{-}, m\right)$ and

$$
\begin{array}{r}
E_{\ell}^{r}\left(\underline{Q_{r}}, q_{r}^{+}, q_{r}^{-}\right) \leqslant E_{\ell}^{r}\left(\underline{v}, v^{+}, v^{-}\right):=\frac{1}{r}\left[\int_{\Omega}|\underline{v}|^{r} \underline{\mathrm{d} x}+\ell \int_{\Omega_{r}^{+}}\left|v^{+}\right|^{r} \mathrm{~d} \underline{x}+\ell \int_{\Omega_{r}^{-}}\left|v^{-}\right|^{r} \mathrm{~d} \underline{x}\right] \\
\forall\left(\underline{v}, v^{+}, v^{-}\right) \in \underline{Y}^{r}\left(f_{r}^{+}, f_{r}^{-}, m\right) ;
\end{array}
$$

that is, a regularized version of $(\mathcal{P})$. 
Lemma 3.1 Let the Assumptions (A1) hold. Then for all $r>1$ and $p=r /(r-1)$, given $\rho \in$ $L_{M}^{r}(\Omega)$ there exists $\underline{Q}_{r}^{\rho} \in \underline{X}^{r}(\rho)$ and

$$
\left\|\underline{Q}_{r}^{\rho}\right\|_{\underline{V}^{r}(\Omega)} \leqslant\left(C_{\Omega}^{*}+1\right)|\rho|_{0, r, \Omega},
$$

where $C_{\Omega}^{*}$ is the constant appearing in 2.12 with $D \equiv \Omega$. Hence it follows that

$$
\inf _{\eta \in L_{M}^{p}(\Omega)} \sup _{\underline{v} \in \underline{V}_{0}^{r}(\Omega)} \frac{(\underline{\nabla} \cdot \underline{v}, \eta)_{\Omega}}{\|\underline{v}\|_{\underline{v}^{r}(\Omega)}|\eta|_{0, p, \Omega}} \geqslant \beta^{-1},
$$

where $\beta=2\left(C_{\Omega}^{*}+1\right)$ and so is independent of $r$.

Proof. See the proof of Lemma 2.1 in [5].

THEOREM 3.1 Let the Assumptions (A1) hold. Then for any given $r \in(1,2]$ there exists a unique solution, $\left(\underline{Q}_{r}, q_{r}^{+}, q_{r}^{-}, u_{r}, \lambda_{r}^{+}, \lambda_{r}^{-}\right) \in \underline{V}_{0}^{r}(\Omega) \times L^{r}\left(\Omega_{r}^{+}\right) \times L^{r}\left(\Omega_{r}^{-}\right) \times L_{M}^{p}(\Omega) \times \mathbb{R} \times \mathbb{R}$, to $\left(\mathcal{P}_{r}\right)$. In addition, we have

$$
\begin{array}{r}
\left\|\underline{Q}_{r}\right\|_{\underline{V}^{r}(\Omega)}^{r}+\ell\left|q_{r}^{+}\right|_{0, r, \Omega_{r}^{+}}^{r}+\ell\left|q_{r}^{-}\right|_{0, r, \Omega_{r}^{-}}^{r} \leqslant C_{\Omega, \ell}\left[\left|f_{r}^{+}\right|_{0, r, \Omega_{r}^{+}}^{r}+\left|f_{r}^{-}\right|_{0, r, \Omega_{r}^{-}}^{r}\right], \\
\left\|u_{r}\right\|_{1, p, \Omega}+\left|\Omega^{+}\right|^{(r-1) / r}\left|\lambda_{r}^{+}\right|+\left|\Omega^{-}\right|^{(r-1) / r}\left|\lambda_{r}^{-}\right| \leqslant C_{\Omega, \ell}\left[\left|f_{r}^{+}\right|_{0, r, \Omega_{r}^{+}}^{r-1}+\left|f_{r}^{-}\right|_{0, r, \Omega_{r}^{-}}^{r-1}\right],
\end{array}
$$

where $p=r /(r-1)$.

Proof. To prove the existence and uniqueness of a solution to $\left(\mathcal{P}_{r}\right)$ and the bounds $(3.9 \mathrm{a} b$ b), we adapt the proof of Theorem 2.2 in [5] for the balanced case, when $m=\left(f_{r}^{ \pm}, 1\right)_{\Omega_{r}^{ \pm}}, q_{r}^{ \pm} \equiv 0$, and $\lambda_{r}^{ \pm}=0$.

Firstly, we define $q_{r}^{f, \pm} \in L^{\infty}(\Omega)$ such that

$$
q_{r}^{f, \pm}:=\left\{\begin{array}{ll}
{\left[\left(f_{r}^{ \pm}, 1\right)_{\Omega_{r}^{ \pm}}-m\right] /\left|\Omega_{r}^{ \pm}\right| \geqslant 0} & \text { in } \Omega_{r}^{ \pm}, \\
0 & \text { in } \Omega \backslash \Omega_{r}^{ \pm},
\end{array} \quad \text { so } \quad\left(f_{r}^{ \pm}-q_{r}^{f, \pm}, 1\right)_{\Omega_{r}^{ \pm}}=m .\right.
$$

It follows from 3.10 that

$$
\left|q_{r}^{f, \pm}\right|_{0, r, \Omega_{r}^{ \pm}} \leqslant\left|f_{r}^{ \pm}\right|_{0, r, \Omega_{r}^{ \pm}} .
$$

Next, we set $\rho:=\left[f_{r}^{+}-q_{r}^{f,+}\right]-\left[f_{r}^{-}-q_{r}^{f,-}\right] \in L_{M}^{r}(\Omega)$. It follows from 3.7) and 3.11 that there exists $\underline{Q}_{r}^{\rho} \in \underline{X}^{r}(\rho)$ and

$$
\left\|\underline{Q}_{r}^{\rho}\right\|_{\underline{V}^{r}(\Omega)} \leqslant\left(C_{\Omega}^{*}+1\right)|\rho|_{0, r, \Omega} \leqslant 2\left(C_{\Omega}^{*}+1\right)\left[\left|f_{r}^{+}\right|_{0, r, \Omega_{r}^{+}}+\left|f_{r}^{-}\right|_{0, r, \Omega_{r}^{-}}\right] .
$$

Therefore, on setting $\widehat{Q}_{r}:=\underline{Q}_{r}-\underline{Q}_{r}^{\rho}$ and $\widehat{q}_{r}^{ \pm}:=q_{r}^{ \pm}-q_{r}^{f, \pm}$, the problem $\left(\mathcal{P}_{r}\right)$, 3.3a-e), can be reduced to: Find $\left(\widehat{Q}_{r}, \widehat{q}_{r}^{+}, \widehat{q}_{r}^{-}\right) \in \underline{Y}^{r}(0,0,0)$ such that

$$
\begin{aligned}
\left(\mid \widehat{Q}_{r}+\right. & \left.\left.\underline{Q}_{r}^{\rho}\right|^{r-2}\left(\widehat{Q}_{r}+\underline{Q}_{r}^{\rho}\right), \underline{v}\right)_{\Omega}+\ell\left(\left|\widehat{q}_{r}^{+}+q_{r}^{f,+}\right|^{r-2}\left(\widehat{q}_{r}^{+}+q_{r}^{f,+}\right), v^{+}\right)_{\Omega_{r}^{+}} \\
& +\ell\left(\left|\widehat{q}_{r}+q_{r}^{f,-}\right|^{r-2}\left(\widehat{q}_{r}+q_{r}^{f,-}\right), v^{-}\right)_{\Omega_{r}^{-}}=0 \quad \forall\left(\underline{v}, v^{+}, v^{-}\right) \in \underline{Y}^{r}(0,0,0) ;
\end{aligned}
$$

which, on recalling [3.6) and [3.1], is the Euler-Lagrange equation for the minimization problem

$$
\inf _{\left(\underline{v}, v^{+}, v^{-}\right) \in \underline{Y}^{r}(0,0,0)} E_{\ell}^{r}\left(\underline{v}+\underline{Q}_{r}^{\rho}, v^{+}+q_{r}^{f,+}, v^{-}+q_{r}^{f,-}\right) .
$$


As $E_{\ell}^{r}(\cdot, \cdot)$ is strictly convex and continuous over the convex set $\underline{Y}^{r}(0,0,0)$, there exists a unique solution $\left(\widehat{Q}_{r}, \widehat{q}_{r}^{+}, \widehat{q}_{r}^{-}\right) \in \underline{Y}^{r}(0,0,0)$ to 3.13 . On setting $\underline{Q}_{r}=\underline{\widehat{Q}}_{r}+\underline{Q}_{r}^{\rho}$ and $q_{r}^{ \pm}=\widehat{q}_{r}^{ \pm}+q_{r}^{f, \pm}$, it follows from 3.13 that $\left(Q_{r}, q_{r}^{+}, q_{r}^{-}\right) \in \underline{Y}^{r}\left(f_{r}^{+}, f_{r}^{-}, m\right)$ is such that

$$
\begin{aligned}
\left(\left|\underline{Q}_{r}\right|^{r-2} \underline{Q}_{r}, \underline{v}\right)_{\Omega}+\ell\left(\left|q_{r}^{+}\right|^{r-2} q_{r}^{+}, v^{+}\right)_{\Omega_{r}^{+}}+\ell\left(\left|q_{r}^{-}\right|^{r-2} q_{r}^{-}, v^{-}\right)_{\Omega_{r}^{-}}=0 \\
\forall\left(\underline{v}, v^{+}, v^{-}\right) \in \underline{Y}^{r}(0,0,0) ;
\end{aligned}
$$

and hence, in particular with $v^{ \pm} \equiv 0$, that

$$
\left(\left|\underline{Q}_{r}\right|^{r-2} \underline{Q}_{r}, \underline{v}\right)_{\Omega}=0 \quad \forall \underline{v} \in \underline{X}^{r}(0) .
$$

It is also easily deduced from $3.3 \mathrm{a}-\mathrm{e})$ and 3.5 that any solution $\left(\underline{Q}_{r}, q_{r}^{ \pm}\right)$of $\left(\mathcal{P}_{r}\right)$ solves $3.15 \mathrm{a}$, and from (3.2) that it is unique. In addition, it follows from (3.14) and (3.5) that

$$
\begin{aligned}
\left|\underline{Q}_{r}\right|_{0, r, \Omega}^{r}+\ell\left|q_{r}^{+}\right|_{0, r, \Omega_{r}^{+}}^{r}+\ell\left|q_{r}^{-}\right|_{0, r, \Omega_{r}^{-}}^{r} & \leqslant\left|\underline{Q}_{r}^{\rho}\right|_{0, r, \Omega}^{r}+\ell\left|q_{r}^{f,+}\right|_{0, r, \Omega_{r}^{+}}^{r}+\ell\left|q_{r}^{f,-}\right|_{0, r, \Omega_{r}^{-}}^{r}, \\
& \left.\underline{\nabla} \cdot \underline{Q_{r}}\right|_{0, r, \Omega} ^{r} \leqslant\left[\left|f_{r}^{+}-q_{r}^{+}\right|_{0, r, \Omega_{r}^{+}}+\left|f_{r}^{-}-q_{r}^{-}\right|_{0, r, \Omega_{r}^{-}}\right]^{r} .
\end{aligned}
$$

The bound (3.9a) follows immediately from 3.16a b), 3.12) and (3.11). Obviously, the introduction of (3.14) is not necessary for proving the existence and uniqueness for 3.6; ; but it is convenient in obtaining the bounds $3.9 \mathrm{a}, \mathrm{b})$.

Let $\mathcal{B} \equiv \underline{\nabla}$. Then it follows from 2.13a b) that $\mathcal{B}: \underline{V}_{0}^{r}(\Omega) \rightarrow\left[L_{M}^{p}(\Omega)\right]^{*}$ and the dual operator $\mathcal{B}^{*}: L_{M}^{p}(\Omega) \rightarrow\left[\underline{V}_{0}^{r}(\Omega)\right]^{*}$. Moreover, it follows from $\sqrt{3.8}$, on noting Lemma I.4.1 and Remark I.4.2 in [18], that $\mathcal{B}^{*}$ is an isomorphism from $L_{M}^{p}(\Omega)$ onto $Z:=\left\{\underline{v}^{*} \in\left[\underline{V}_{0}^{r}(\Omega)\right]^{*}:\left\langle\underline{v}^{*}, \underline{v}\right\rangle_{\underline{v}_{0}^{r}(\Omega)}=0\right.$ $\left.\forall \underline{v} \in \operatorname{ker}(\mathcal{B}) \subset \underline{V}_{0}^{r}(\Omega)\right\}$, where $\langle\cdot, \cdot\rangle_{\underline{V}_{0}^{r}(\Omega)}$ is the duality pairing on $\left[\underline{V}_{0}^{r}(\Omega)\right]^{*} \times \underline{V}_{0}^{r}(\Omega)$. Hence it follows from $3.15 \mathrm{~b}$, as $\operatorname{ker}(\mathcal{B}) \equiv \underline{X}^{r}(0)$, that there exists a unique $u_{r} \in L_{M}^{p}(\Omega)$ satisfying 3.3ap. In addition, from $(3.8)$ and $3.3 \mathrm{a}$ we have

$$
\left|u_{r}\right|_{0, p, \Omega} \leqslant \beta \sup _{\underline{v} \in \underline{V}_{0}^{r}(\Omega)} \frac{\left(\left|\underline{Q}_{r}\right|^{r-2} \underline{Q}_{r}, \underline{v}\right)_{\Omega}}{\| \underline{v}_{\underline{V}^{r}(\Omega)}} \leqslant \beta\left|\underline{Q}_{r}\right|_{0, r, \Omega}^{r-1} .
$$

Choosing $v^{\mp} \equiv 0$ in 3.15a, we have $v^{ \pm}=\mp \underline{\nabla} . \underline{v}$; and hence, on noting 3.3a, it follows that $\ell\left|q_{r}^{ \pm}\right|^{r-2} q_{r}^{ \pm} \mp u_{r}$ are constant on $\Omega_{r}^{ \pm}$. On choosing

$$
\lambda_{r}^{ \pm}=-\ell\left|q_{r}^{ \pm}\right|^{r-2} q_{r}^{ \pm} \pm u_{r}
$$

we see that $3.3 \mathrm{~b}, \mathrm{c})$ hold for general $v^{ \pm} \in L^{r}\left(\Omega_{r}^{ \pm}\right)$. We have thus proved that there exists a unique solution to $\left(\mathcal{P}_{r}\right),(3.3 \mathrm{a}-\mathrm{e})$, which is equivalent to the minimization problem 3.6. Furthermore, we deduce from 3.18 that

$$
\left|\lambda_{r}^{ \pm}\right| \leqslant\left|\Omega_{r}^{ \pm}\right|^{-(r-1) / r}\left[\ell\left|q_{r}^{ \pm}\right|_{0, r, \Omega_{r}^{ \pm}}^{r-1}+\left|u_{r}\right|_{0, p, \Omega}\right] .
$$

Finally, it follows from $3.3 \mathrm{a}$ that

$$
\left|\left(u_{r}, \underline{\nabla} \cdot \underline{v}\right)_{\Omega}\right|=\left|\left(\left|\underline{Q}_{r}\right|^{r-2} \underline{Q}_{r}, \underline{v}\right)_{\Omega}\right| \leqslant\left|\underline{Q}_{r}\right|_{0, r, \Omega}^{r-1}|\underline{v}|_{0, r, \Omega} \quad \forall \underline{v} \in \underline{V}_{0}^{r}(\Omega) .
$$

Therefore noting that $\Omega^{ \pm} \subseteq \Omega_{r}^{ \pm}$, and combining 3.17, 3.9a, 3.19) and 3.20) immediately yield the desired result $3.9 \mathrm{~b}$. 
Next we are more precise about our choice of regularized data $f_{r}^{ \pm}$for $\left(\mathcal{P}_{r}\right)$. With $f^{ \pm} \in \mathcal{M}^{+}\left(\overline{\Omega^{ \pm}}\right)$ being the data for problem $(\mathcal{P})$, we choose, for any $r>1$, corresponding regularized data $f_{r}^{ \pm}$as follows. If $f^{ \pm} \in L^{r}\left(\Omega^{ \pm}\right)$we set $f_{r}^{ \pm} \equiv f^{ \pm}$, whereas if $\overline{\Omega^{ \pm}} \subset \Omega$ we set

$$
f_{r}^{ \pm}(\underline{x}):=\frac{1}{|B(\underline{x}, r-1)|} \int_{B(\underline{x}, r-1)} f^{ \pm} \quad \forall \underline{x} \in \Omega,
$$

where $B(\underline{x}, \rho)$ is the closed ball in $\mathbb{R}^{n}$ centred at $\underline{x}$ with radius $\rho>0$ and $f^{ \pm}$is extended from $\overline{\Omega^{ \pm}}$ to $\mathbb{R}^{n}$ by zero. It follows for $r-1$ sufficiently small that

$$
\begin{aligned}
& \quad \overline{\Omega^{ \pm}} \subseteq \overline{\Omega_{r}^{ \pm}}:=\overline{\Omega^{ \pm}} \cup \operatorname{supp}\left(f_{r}^{ \pm}\right) \subseteq \bar{\Omega}, \quad f_{r}^{ \pm} \in L^{r}\left(\Omega_{r}^{ \pm}\right), \\
& \text {with } f_{r}^{ \pm} \geqslant 0 \quad \text { a.e. in } \Omega \quad \text { and }\left(f_{r}^{ \pm}, 1\right)_{\Omega_{r}^{ \pm}}=\int_{\overline{\Omega^{ \pm}}} f^{ \pm} ;
\end{aligned}
$$

moreover,

$$
\begin{aligned}
& \lim _{r \rightarrow 1}\left|\Omega_{r}^{ \pm} \backslash \Omega^{ \pm}\right|=0, \quad \limsup _{r \rightarrow 1}\left|f_{r}^{ \pm}\right|_{0, r, \Omega_{r}^{ \pm}}^{r} \leqslant \int_{\Omega^{ \pm}}\left|f^{ \pm}\right|, \\
& f_{r}^{ \pm} \rightarrow f^{ \pm} \quad \text { vaguely in } \mathcal{M}(\bar{\Omega}) \quad \text { as } r \rightarrow 1 .
\end{aligned}
$$

For general $f^{ \pm} \in \mathcal{M}^{+}(\bar{\Omega})$, the construction 3.21 can be modified so that $3.22 \mathrm{a}$ b) still hold. For example, one can partition $\Omega$ into a finite number of strictly star-shaped sets $D_{j}$, with "centres" $\underline{x}_{j}$ and outward unit normals $\underline{v}_{j}$ on their boundaries $\partial D_{j}$, so that $\left(\underline{y}-\underline{x}_{j}\right) \cdot \underline{v}_{j} \geqslant c>0$ for a.e. $\underline{y} \in \partial D_{j}$. Then employ the local change of variable $\underline{\tau}_{t}(\underline{x})=\underline{x}_{j}+t\left(\underline{x}-\underline{x}_{j}\right)$ for $t \in(0,1)$, inducing a push forward measure, before applying the mollification (3.21); for details see the proof of Lemma 2.4 in [5], where these techniques are used.

In the theorem below, we will show subsequence convergence, as $r \rightarrow 1$, of the unique solution of $\left(\mathcal{P}_{r}\right)$, with the above choice of data $f_{r}^{ \pm}$, to a solution of:

$(\widehat{\mathcal{P}})$ Find $\underline{Q} \in \underline{V}_{0}^{\mathcal{M}}(\Omega), q^{ \pm} \in \mathcal{M}\left(\overline{\Omega^{ \pm}}\right), u \in C_{M}(\bar{\Omega})$ and $\lambda^{ \pm} \in \mathbb{R}$ such that

$$
\begin{array}{ll}
\int_{\bar{\Omega}}|\underline{v}|-\int_{\bar{\Omega}}|\underline{Q}| \geqslant\langle\underline{\nabla} \cdot(\underline{v}-\underline{Q}), u\rangle_{C(\bar{\Omega})} & \forall \underline{v} \in \underline{V}_{0}^{\mathcal{M}}(\Omega), \\
\ell\left[\int_{\overline{\Omega^{+}}}\left|v^{+}\right|-\int_{\overline{\Omega^{+}}}\left|q^{+}\right|\right] \geqslant\left\langle v^{+}-q^{+}, u-\lambda^{+}\right\rangle_{C\left(\overline{\Omega^{+}}\right)} & \forall v^{+} \in \mathcal{M}\left(\overline{\Omega^{+}}\right), \\
\ell\left[\int_{\overline{\Omega^{-}}}\left|v^{-}\right|-\int_{\overline{\Omega^{-}}}\left|q^{-}\right|\right] \geqslant-\left\langle v^{-}-q^{-}, u+\lambda^{-}\right\rangle_{C\left(\overline{\Omega^{-}}\right)} & \forall v^{-} \in \mathcal{M}\left(\overline{\Omega^{-}}\right), \\
\langle\underline{\nabla} \cdot \underline{Q}, \eta\rangle_{C(\bar{\Omega})}=\left\langle f^{+}-q^{+}, \eta\right\rangle_{C\left(\overline{\Omega^{+}}\right)}-\left\langle f^{-}-q^{-}, \eta\right\rangle_{C\left(\overline{\Omega^{-}}\right)} & \forall \eta \in C(\bar{\Omega}), \\
\int_{\overline{\Omega^{+}}}\left(f^{+}-q^{+}\right)=\int_{\overline{\Omega^{-}}}\left(f^{-}-q^{-}\right)=m . &
\end{array}
$$

We now introduce the measure analogues of 3.4 and 3.5. For $\rho \in \mathcal{M}_{M}(\bar{\Omega}):=\{\mu \in \mathcal{M}(\bar{\Omega})$ : $\left.\int_{\bar{\Omega}} \mu=0\right\}$, let

$$
\underline{X}^{\mathcal{M}}(\rho):=\left\{\underline{v} \in \underline{V}_{0}^{\mathcal{M}}(\Omega):\langle\underline{\nabla} \cdot \underline{v}, \eta\rangle_{C(\bar{\Omega})}=\langle\rho, \eta\rangle_{C(\bar{\Omega})} \forall \eta \in C(\bar{\Omega})\right\},
$$


and

$$
\begin{gathered}
\underline{Y}^{\mathcal{M}}\left(f^{+}, f^{-}, m\right):=\left\{\underline{v}, v^{+}, v^{-}\right) \in \underline{X}^{\mathcal{M}}\left(\left[f^{+}-v^{+}\right]-\left[f^{-}-v^{-}\right]\right) \times \mathcal{M}\left(\overline{\Omega^{+}}\right) \times \mathcal{M}\left(\overline{\Omega^{-}}\right): \\
\left.\int_{\overline{\Omega^{+}}}\left(f^{+}-v^{+}\right)=\int{\overline{\Omega^{-}}}\left(f^{-}-v^{-}\right)=m\right\} .
\end{gathered}
$$

It follows from 3.23a-e), 3.24 and 3.25 that a solution of $(\widehat{\mathcal{P}})$ is such that $\left(\underline{Q}, q^{+}, q^{-}\right) \in$ $\underline{Y}^{\mathcal{M}}\left(f^{+}, f^{-}, m\right)$ and

$$
\begin{aligned}
E_{\ell}^{\mathcal{M}}\left(\underline{Q}, q^{+}, q^{-}\right) \leqslant E_{\ell}^{\mathcal{M}}\left(\underline{v}, v^{+}, v^{-}\right):=\int_{\bar{\Omega}}|\underline{v}|+ & \ell \int_{\overline{\Omega^{+}}}\left|v^{+}\right|+\ell \int_{\overline{\Omega^{-}}}\left|v^{-}\right| \\
& \forall\left(\underline{v}, v^{+}, v^{-}\right) \in \underline{Y}^{\mathcal{M}}\left(f^{+}, f^{-}, m\right) ;
\end{aligned}
$$

that is $(\mathcal{P}), \sqrt{2.7}$, but with the support of $q^{ \pm}$restricted to $\overline{\Omega^{ \pm}}$. Hence, proving existence for $(\widehat{\mathcal{P}})$ yields existence for $(\mathcal{P})$ too. However, as the solution of $(\mathcal{P})$ is possibly not unique, we do not have the equivalence of the problems $(\widehat{\mathcal{P}})$ and $(\mathcal{P})$.

TheOREM 3.2 Let the Assumptions (A1) hold. Then there exists a subsequence $\left\{\left(\underline{Q}_{r_{j}}\right.\right.$, $\left.\left.q_{r_{j}}^{ \pm}, u_{r_{j}}, \lambda_{r_{j}}^{ \pm}\right)\right\}_{r_{j}>1}$ of $\left\{\left(\underline{Q}_{r}, q_{r}^{ \pm}, u_{r}, \lambda_{r}^{ \pm}\right)\right\}_{r>1}$, where $\left(\underline{Q}_{r}, q_{r}^{+}, q_{r}^{-}, u_{r}, \lambda_{r}^{+}, \lambda_{r}^{-}\right) \in \underline{V}_{0}^{r}(\Omega) \times L^{r}\left(\bar{\Omega}_{r}^{+}\right)$ $\times L^{r}\left(\Omega_{r}^{-}\right) \times L_{M}^{p}(\Omega) \times \mathbb{R} \times \mathbb{R}$ is the unique solution of $\left(\mathcal{P}_{r}\right)$ with $f_{r}^{ \pm}$satisfying $\left.3.22 \mathrm{a} b \mathrm{~b}\right)$, such that as $r_{j} \rightarrow 1$,

$$
\begin{aligned}
\underline{Q}_{r_{j}} & \rightarrow \underline{Q} & & \text { vaguely in }[\mathcal{M}(\bar{\Omega})]^{n}, \\
\underline{\nabla} \cdot \underline{Q}_{r_{j}} & \rightarrow \underline{\nabla} \cdot \underline{Q} & & \text { vaguely in } \mathcal{M}(\bar{\Omega}), \\
q_{r_{j}}^{ \pm} & \rightarrow q^{ \pm} & & \text {vaguely in } \mathcal{M}(\bar{\Omega}), \\
u_{r_{j}} & \rightarrow u & & \text { strongly in } C(\bar{\Omega}), \\
\lambda_{r_{j}}^{ \pm} & \rightarrow \lambda^{ \pm} . & &
\end{aligned}
$$

Moreover, $\left(\underline{Q}, q^{+}, q^{-}, u, \lambda^{+}, \lambda^{-}\right) \in \underline{V}_{0}^{\mathcal{M}}(\Omega) \times \mathcal{M}\left(\overline{\Omega^{+}}\right) \times \mathcal{M}\left(\overline{\Omega^{-}}\right) \times C_{M}(\bar{\Omega}) \times \mathbb{R} \times \mathbb{R}$ solves $(\widehat{\mathcal{P}}), 3.23 \mathrm{a}-\mathrm{e})$.

Proof. It follows from 3.9a b) and 3.22a b) that for all $r$ sufficiently close to 1 ,

$$
\left|\underline{Q}_{r}\right|_{0,1, \Omega}+\left|\underline{\nabla} \cdot \underline{Q}_{r}\right|_{0,1, \Omega}+\left|q_{r}^{ \pm}\right|_{0,1, \Omega_{r}^{+}}+\left\|u_{r}\right\|_{1, p^{*}, \Omega}+\left|\lambda_{r}^{ \pm}\right| \leqslant C_{\Omega, \Omega^{ \pm}, \ell, f^{ \pm}},
$$

where $p^{*}>n$. The subsequence convergence results $3.27 \mathrm{a}$-e), where $\left\{Q, q^{ \pm}, u, \lambda^{ \pm}\right\} \in \underline{V}_{0}^{\mathcal{M}}(\Omega) \times$ $\mathcal{M}(\bar{\Omega}) \times \mathcal{M}(\bar{\Omega}) \times C(\bar{\Omega}) \times \mathbb{R} \times \mathbb{R}$, then follow immediately from 3.28,, 2.9 ) and noting the compact Sobolev embedding $W^{1, p^{*}}(\Omega) \hookrightarrow \hookrightarrow C(\bar{\Omega})$. As $\left(u_{r}, 1\right)_{\Omega}=0$ for all $r>1$, and recalling 3.1), it follows that the limits $\left\{q^{ \pm}, u\right\}$ are in $\mathcal{M}\left(\overline{\Omega^{ \pm}}\right) \times C_{M}(\bar{\Omega})$.

Passing to the $r_{j} \rightarrow 1$ limit in the $r_{j}$ versions of $3.3 \mathrm{~d}$, with $\eta \in C(\bar{\Omega})$ and $3.3 \mathrm{e}$ shows, on noting $3.27 \mathrm{~b}$ c) and (3.22b), that $3.23 \mathrm{~d} . \mathrm{e})$ hold.

For any $\underline{\xi} \in\left[C_{0}^{\infty}(\Omega)\right]^{n}$, we choose $\underline{v}=\underline{\xi}-\underline{Q}_{r_{j}}$ in the $r_{j}$ version of $3.3 \mathrm{a}$ to deduce, on noting [3.1], that

$$
\left(u_{r_{j}}, \underline{\nabla} \cdot\left(\underline{Q}_{r_{j}}-\underline{\xi}\right)\right)_{\Omega}=\left(\left|\underline{Q}_{r_{j}}\right|^{r_{j}-2} \underline{Q}_{r_{j}}, \underline{Q}_{r_{j}}-\underline{\xi}\right)_{\Omega} \geqslant \frac{1}{r_{j}} \int_{\Omega}\left|\underline{Q}_{r_{j}}\right|^{r_{j}} \mathrm{~d} \underline{x}-\left.\frac{1}{r_{j}} \int_{\Omega} \underline{\mid \xi}\right|^{r_{j}} \mathrm{~d} \underline{x} .
$$


For all $\underline{\xi} \in\left[C_{0}^{\infty}(\Omega)\right]^{n}$, we have

$$
\frac{1}{r} \int_{\Omega}|\underline{\xi}|^{r} \mathrm{~d} \underline{x} \rightarrow \int_{\Omega}|\underline{\xi}| \mathrm{d} \underline{x} \quad \text { as } r \rightarrow 1
$$

and it follows from $3.27 \mathrm{~b}$ ) that

$$
\left(\underline{\nabla} \cdot\left(\underline{Q}_{r_{j}}-\underline{\xi}\right), u_{r_{j}}\right)_{\Omega} \rightarrow\langle\underline{\nabla} \cdot(\underline{Q}-\underline{\xi}), u\rangle_{C(\bar{\Omega})} \quad \text { as } r_{j} \rightarrow 1 .
$$

Next we note from $3.27 \mathrm{a}$ ) and 2.16 that

$$
\liminf _{r_{j} \rightarrow 1} \frac{1}{r_{j}} \int_{\Omega}\left|\underline{Q}_{r_{j}}\right|^{r_{j}} \mathrm{~d} \underline{x} \geqslant \liminf _{r_{j} \rightarrow 1} \int_{\Omega}\left|\underline{Q}_{r_{j}}\right| \mathrm{d} \underline{x} \geqslant \int_{\bar{\Omega}}|\underline{Q}| .
$$

Combining $3.29-3.32$ yields

$$
\int_{\bar{\Omega}}|\underline{\xi}|-\int_{\bar{\Omega}}|\underline{Q}| \geqslant\langle\underline{\nabla} \cdot(\underline{\xi}-\underline{Q}), u\rangle_{C \bar{\Omega})} \quad \forall \underline{\xi} \in\left[C_{0}^{\infty}(\Omega)\right]^{n} .
$$

Noting (2.17a b) yields the desired result (3.23a).

Similarly, using $3.27 \mathrm{c}-\mathrm{e})$ one can pass to the limit in the $r_{j}$ versions of $3.3 \mathrm{~b}$ c) with $v^{ \pm}=$ $\xi^{ \pm}-q_{r_{j}}^{ \pm}$, for any $\xi^{ \pm} \in C_{0}^{\infty}\left(\Omega^{ \pm}\right)$, to obtain $3.23 \mathrm{~b}$ c) with $v^{ \pm}=\xi^{ \pm}$. Noting 2.18 then yields the desired results $3.23 \mathrm{~b}$ c). Hence $\left(\underline{Q}, q^{ \pm}, u, \lambda^{ \pm}\right) \in \underline{V}_{0}^{\mathcal{M}}(\Omega) \times \mathcal{M}\left(\overline{\Omega^{+}}\right) \times \mathcal{M}\left(\overline{\Omega^{-}}\right) \times C_{M}(\bar{\Omega}) \times \mathbb{R} \times \mathbb{R}$ solves $(\widehat{\mathcal{P}})$.

In the next two subsections, we adapt the arguments above for $(\mathcal{P})$ to show the existence for $(\mathcal{U})$, 2.10, and $(\mathcal{P M}), 2.11$, respectively. In both cases, we just briefly state the key differences.

\subsection{Existence for $(\mathcal{U})$}

As we saw in Section 2 if we solve $(\mathcal{P}), 2.7$, with $\int_{\overline{\Omega^{+}}} f^{+}=m<\int \overline{\Omega^{-}} f^{-}$then $q^{+} \equiv 0$, and similarly if $\int \overline{\Omega^{-}} f^{\underline{-}}=m<\int \frac{\Omega_{\Omega^{+}}}{f^{+}}$then $q^{-} \equiv 0$. Hence existence of a solution to $(\mathcal{U})$, 2.10), follows immediately from our existence proof for $(\mathcal{P})$ above. However, as our numerical approximation of $(\mathcal{U})$ is based on the discretization of a regularized problem $\left(\mathcal{U}_{r}\right)$ for $r>1$, we now show subsequence convergence, as $r \rightarrow 1$, of the unique solution of $\left(\mathcal{U}_{r}\right)$ to a solution of $(\mathcal{U})$.

We shall assume here, in addition to the assumptions (A1), that $\Omega$ is star-shaped and $f^{ \pm} \in$ $L^{r_{0}}\left(\Omega^{ \pm}\right)$for some $r_{0}>1$. Let $f:=f^{+}-f^{-}$.

For a given $r \in\left(1, r_{0}\right)$, we then consider the following problem:

$\left(\mathcal{U}_{r}\right)$ Find $\underline{Q}_{r} \in \underline{V}_{0}^{r}(\Omega)$ such that

$$
\left(\left|\underline{Q}_{r}\right|^{r-2} \underline{Q}_{r}, \underline{v}\right)_{\Omega}+\ell\left(\left|\underline{\nabla} \cdot \underline{Q}_{r}-f\right|^{r-2}\left(\underline{\nabla} \cdot \underline{Q}_{r}-f\right), \underline{\nabla} \cdot \underline{v}\right)_{\Omega}=0 \quad \forall \underline{v} \in \underline{V}_{0}^{r}(\Omega) .
$$

$\left(\mathcal{U}_{r}\right)$ is the Euler-Lagrange equation for the following strictly convex minimization problem: Find $\underline{Q}_{r} \in \underline{V}_{0}^{r}(\Omega)$ such that

$$
E_{\ell}^{r}\left(\underline{Q}_{r}\right) \leqslant E_{\ell}^{r}(\underline{v}):=\frac{1}{r} \int_{\Omega}\left[|\underline{v}|^{r}+\ell|\underline{\nabla} \cdot \underline{v}-f|^{r}\right] \mathrm{d} \underline{x} \quad \forall \underline{v} \in \underline{V}_{0}^{r}(\Omega) .
$$


Hence, there exists a unique solution $\underline{Q}_{r} \in \underline{V}_{0}^{r}(\Omega)$ to $\left(\mathcal{U}_{r}\right), 3.34$ and 3.35 are equivalent problems; and moreover,

$$
\left|\underline{Q}_{r}\right|_{0, r, \Omega}^{r}+\ell\left|\underline{\nabla} \cdot \underline{Q}_{r}-f\right|_{0, r, \Omega}^{r} \leqslant \ell|f|_{0, r, \Omega}^{r} .
$$

It follows from 3.36 that there exists $\underline{Q} \in \underline{V}_{0}^{\mathcal{M}}(\Omega)$ such that there exists a subsequence $\left\{\underline{Q}_{r_{j}}\right\}_{r_{j}>1}$, where $Q_{r}$ is the unique solution of $\left(\mathcal{U}_{r}\right)$, such that $\left.3.27 \mathrm{a}, \mathrm{b}\right)$ hold as $r_{j} \rightarrow 1$.

For any $\underline{\xi} \in\left[C_{0}^{\infty}(\Omega)\right]^{n}$, we have $E_{\ell}^{r_{j}}(\underline{\xi}) \geqslant E_{\ell}^{r_{j}}\left(\underline{Q}_{r_{j}}\right)$. Similarly to 3.32 , we conclude on noting $3.27 \mathrm{a} b$ ) and 2.16 that

$$
\liminf _{r_{j} \rightarrow 1} E_{\ell}^{r_{j}}\left(\underline{Q}_{r_{j}}\right) \geqslant \int_{\bar{\Omega}}[|\underline{Q}|+\ell|\underline{\nabla} \cdot \underline{Q}-f|] .
$$

Similarly to 3.30 , we have

$$
\limsup _{r_{j} \rightarrow 1} E_{\ell}^{r_{j}}(\underline{\xi}) \leqslant \int_{\bar{\Omega}}[|\underline{\xi}|+\ell|\underline{\nabla} \cdot \underline{\xi}-f|]
$$

Hence, it follows on combining the above that $\underline{Q} \in \underline{V}_{0}^{\mathcal{M}}(\Omega)$ is such that

$$
\int_{\bar{\Omega}}[|\underline{\xi}|+\ell|\underline{\nabla} \cdot \underline{\xi}-f|] \geqslant \int_{\bar{\Omega}}[|\underline{Q}|+\ell|\underline{\nabla} \cdot \underline{Q}-f|] \quad \forall \underline{\xi} \in\left[C_{0}^{\infty}(\Omega)\right]^{n} .
$$

Without loss of generality, we assume that the "centre" of the star-shaped $\Omega$ is the origin, and set $d:=\operatorname{dist}(\underline{0}, \partial \Omega)$. Let $j \in C^{\infty}\left(\mathbb{R}^{n}\right)$, with compact support in $B(\underline{0}, 1)$, be such that

$$
\int_{B(\underline{0}, 1)} j(\underline{x}) \mathrm{d} \underline{x}=1, \quad j(\underline{x}) \geqslant 0 \quad \text { and } \quad j(-\underline{x})=j(\underline{x}) .
$$

For any $\varepsilon>0$, let $J_{\varepsilon}: \mathcal{M}\left(\mathbb{R}^{n}\right) \rightarrow C_{0}^{\infty}\left(\mathbb{R}^{n}\right)$ be such that

$$
\left(J_{\varepsilon} \eta\right)(\underline{x})=\left\langle\eta(\cdot), j_{\varepsilon}(\underline{x}-\cdot)\right\rangle_{C\left(\mathbb{R}^{n}\right)} \quad \forall \underline{x} \in \mathbb{R}^{n},
$$

where $j_{\varepsilon}(\underline{x})=\varepsilon^{-n} j\left(\varepsilon^{-1} \underline{x}\right)$; and let $\underline{\tau}_{\varepsilon}: \mathbb{R}^{n} \rightarrow \mathbb{R}^{n}$ be such that $\left.\underline{\tau}_{\varepsilon} \underline{x}\right)=(1+\varepsilon)^{-1} \underline{x}$ inducing the push forward $\underline{\tau}_{\varepsilon \#}: \mathcal{M}\left(\mathbb{R}^{n}\right) \rightarrow \mathcal{M}\left(\mathbb{R}^{n}\right)$ (see e.g. [2, p. 32]). We extend $J_{\varepsilon}$ and $\underline{\tau}_{\varepsilon \#}$ in the natural way, so that $\underline{J}_{\varepsilon}:\left[\mathcal{M}\left(\mathbb{R}^{n}\right)\right]^{n} \rightarrow\left[C_{0}^{\infty}\left(\mathbb{R}^{n}\right)\right]^{n}$ and $\underline{\tau}_{\varepsilon \#}:\left[\mathcal{M}\left(\mathbb{R}^{n}\right)\right]^{n} \rightarrow\left[\mathcal{M}\left(\mathbb{R}^{n}\right)\right]^{n}$. It follows for any $v \in \mathcal{M}(\bar{\Omega})$ that $v_{k}:=J_{\frac{d}{3 k}}\left(\underline{\tau}_{\frac{1}{k} \#} v\right) \in C_{0}^{\infty}(\Omega)$ and $\left\{v_{k}\right\}_{k} \geqslant 1$ satisfy 2.18$)$ as $k \rightarrow \infty$; and for any $\underline{v} \in \underline{V}_{0}^{\mathcal{M}}(\Omega)$ that $\underline{v}_{k}:=\underline{J}_{\frac{d}{3 k}}\left(\underline{\tau}_{\frac{1}{k}} \# \underline{v}\right) \in\left[C_{0}^{\infty}(\Omega)\right]^{n}$ and $\left\{\underline{v}_{k}\right\}_{k} \geqslant 1$ satisfy $2.17 \mathrm{a}$ b) as $k \rightarrow \infty$. We note that

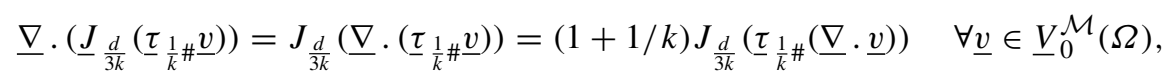

and

$$
\int_{\Omega}\left|J_{\frac{d}{3 k}}\left(\tau_{\frac{1}{k} \#} v\right)\right| \mathrm{d} \underline{x} \leqslant \int_{\bar{\Omega}}\left|\underline{\tau}_{\frac{1}{k} \#} v\right| \leqslant \int_{\bar{\Omega}}|v| \quad \forall v \in \mathcal{M}(\bar{\Omega}) .
$$

In addition, we note that for any $r \in[1, \infty)$ and any $v \in L^{r}(\Omega)$,

$$
\left|v-J_{\frac{d}{3 k}}\left(\underline{\tau}_{\frac{1}{k} \#} v\right)\right|_{0, r, \Omega} \rightarrow 0 \quad \text { as } \quad k \rightarrow \infty .
$$


For any $\underline{v} \in \underline{V}_{0}^{\mathcal{M}}(\Omega)$, we then choose $\underline{\xi} \equiv \underline{J}_{\frac{d}{3 k}}\left(\underline{\tau}_{\frac{1}{k}} \# \underline{v}\right)$ in 3.39 . It follows from $2.17 \mathrm{~b},, 3.40$, (3.41) and 3.42 that

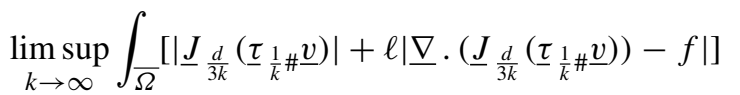

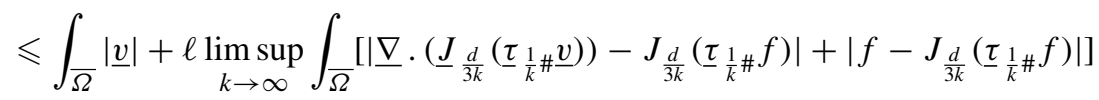

$$
\begin{aligned}
& \leqslant \int_{\bar{\Omega}}|\underline{v}|+\ell \limsup _{k \rightarrow \infty} \int_{\bar{\Omega}}|(1+1 / k)(\underline{\nabla} \cdot \underline{v})-f| \leqslant \int_{\bar{\Omega}}[|\underline{v}|+\ell|\underline{\nabla} \cdot \underline{v}-f|] \text {. }
\end{aligned}
$$

Hence, a solution to

(U) Find $\underline{Q} \in \underline{V}_{0}^{\mathcal{M}}(\Omega)$ such that

$$
\int_{\bar{\Omega}}[|\underline{v}|+\ell|\underline{\nabla} \cdot \underline{v}-f|] \geqslant \int_{\bar{\Omega}}[|\underline{Q}|+\ell|\underline{\nabla} \cdot \underline{Q}-f|] \quad \forall \underline{v} \in \underline{V}_{0}^{\mathcal{M}}(\Omega),
$$

that is, 2.10, exists (which is already known for more general data via $(\mathcal{P})$ ) and, moreover, is the limit of solutions to the regularized problems $\left(\mathcal{U}_{r}\right)$ as $r \rightarrow 1$.

\subsection{Existence for $(\mathcal{P} \mathcal{M})$}

Similarly to the previous subsection, we shall assume here, in addition to the assumptions (A1), that $\Omega$ is star-shaped and that the nonnegative $f^{(i)}, i=1 \rightarrow I$, for some $r_{0}>1$ are in $L^{r_{0}}(\Omega)$. In addition, we assume that $m \in\left(0, \min _{i=1 \rightarrow I}\left\{\int_{\bar{\Omega}} f^{(i)}\right\}\right]$, and $\Omega_{g}$ is an open Lipschitz subset of $\Omega$. Moreover, we will consider the simplified problem, as mentioned previously in Section 2 , and assume that for a given constant $G \geqslant m /\left|\Omega_{g}\right|$,

$$
g \in K_{G}(m):=\left\{\eta \in K_{G}: \int_{\Omega_{g}} \eta \mathrm{d} \underline{x}=m\right\}
$$

where

$$
K_{G}:=\left\{\eta \in L^{\infty}\left(\Omega_{g}\right): \eta \in[0, G] \text { a.e. in } \Omega_{g}\right\} ;
$$

as opposed to $g \in \mathcal{M}^{+}\left(\bar{\Omega}_{g}\right)$, with $\int_{\bar{\Omega}_{g}} g=m$.

For a given $r \in\left(1, r_{0}\right)$, we then consider the following problem:

$\left(\mathcal{P} \mathcal{M}_{r}\right)$ Find $\underline{Q}_{r}^{(i)} \in \underline{V}_{0}^{r}(\Omega), i=1 \rightarrow I$, and $g_{r} \in K_{G}(m)$ such that

$$
\begin{gathered}
\left(\left|\underline{Q}_{r}^{(i)}\right|^{r-2} \underline{Q}_{r}^{(i)}, \underline{v}\right)_{\Omega}+\ell\left(\left|\underline{\nabla} \cdot \underline{Q}_{r}^{(i)}-f^{(i)}+g_{r}\right|^{r-2}\left(\underline{\nabla} \cdot \underline{Q}_{r}^{(i)}-f^{(i)}+g_{r}\right), \underline{\nabla} \cdot \underline{v}\right)_{\Omega}=0 \\
\forall \underline{v} \in \underline{V}_{0}^{r}(\Omega), i=1 \rightarrow I, \\
\sum_{i=1}^{I}\left(\left|\underline{\nabla} \cdot \underline{Q}_{r}^{(i)}-f^{(i)}+g_{r}\right|^{r-2}\left(\underline{\nabla} \cdot \underline{Q}_{r}^{(i)}-f^{(i)}+g_{r}\right), \eta-g_{r}\right)_{\Omega_{g}} \geqslant 0 \\
\forall \eta \in K_{G}(m) .
\end{gathered}
$$


$\left(\mathcal{P} \mathcal{M}_{r}\right)$ is the Euler-Lagrange system for the following strictly convex minimization problem: Find $\underline{Q}_{r}^{(i)} \in \underline{V}_{0}^{r}(\Omega), i=1 \rightarrow I$, and $g_{r} \in K_{G}(m)$ such that

$$
\begin{aligned}
E_{\ell}^{r}\left(\left\{\underline{Q}_{r}^{(i)}\right\}_{i=1}^{I}, g_{r}\right) \leqslant E_{\ell}^{r}\left(\left\{\underline{v}^{(i)}\right\}_{i=1}^{I}, \eta\right):= & \frac{1}{r} \sum_{i=1}^{I} \int_{\Omega}\left[\left|\underline{v}^{(i)}\right|^{r}+\ell\left|\underline{\nabla} \cdot \underline{v}^{(i)}-f^{(i)}+\eta\right|^{r}\right] \mathrm{d} \underline{x} \\
& \forall \underline{v}^{(i)} \in \underline{V}_{0}^{r}(\Omega), i=1 \rightarrow I, \forall \eta \in K_{G}(m) .
\end{aligned}
$$

Hence, there exists a unique solution $\left(\left\{\underline{Q}_{r}^{(i)}\right\}_{i=1}^{I}, g_{r}\right)$ to $\left(\mathcal{P} \mathcal{M}_{r}\right), 3.46 \mathrm{a}$ b) and 3.47 are equivalent problems; and moreover,

$$
\sum_{i=1}^{I}\left[\left|\underline{Q}_{r}^{(i)}\right|_{0, r, \Omega}^{r}+\ell\left|\underline{\nabla} \cdot \underline{Q}_{r}^{(i)}-f^{(i)}+g_{r}\right|_{0, r, \Omega}^{r}\right] \leqslant \ell \sum_{i=1}^{I}\left|\frac{m}{\left|\Omega_{g}\right|}-f^{(i)}\right|_{0, r, \Omega}^{r} .
$$

It follows from 3.48 that there exist $\underline{Q}^{(i)} \in \underline{V}_{0}^{\mathcal{M}}(\Omega), i=1 \rightarrow I$, and $g \in K_{G}(m)$ such that there exists a subsequence $\left\{\left\{\underline{Q}_{r_{j}}^{(i)}\right\}_{i=1}^{I}, g_{r_{j}}\right\}_{r_{j}>1}$, where $\left(\left\{\underline{Q}_{r}^{(i)}\right\}_{i=1}^{I}, g_{r}\right)$ is the unique solution of $\left(\mathcal{P} \mathcal{M}_{r}\right)$, such that

$$
\begin{aligned}
& \underline{Q}_{r_{j}}^{(i)} \rightarrow \underline{Q}^{(i)} \quad \text { vaguely in }[\mathcal{M}(\bar{\Omega})]^{n}, \quad i=1 \rightarrow I, \\
& \underline{\nabla} \cdot \underline{Q}_{r_{j}}^{(i)} \rightarrow \underline{\nabla} \cdot \underline{Q}^{(i)} \quad \text { vaguely in } \mathcal{M}(\bar{\Omega}), \quad i=1 \rightarrow I, \\
& g_{r_{j}} \rightarrow g \quad \text { vaguely in } L^{\infty}\left(\Omega_{g}\right)
\end{aligned}
$$

as $r_{j} \rightarrow 1$.

For any $\underline{\xi}^{(i)} \in\left[C_{0}^{\infty}(\Omega)\right]^{n}, i=1 \rightarrow I$, and $\eta \in K_{g}(m)$, we have $E_{\ell}^{r_{j}}\left(\left\{\underline{\xi}^{(i)}\right\}_{i=1}^{I}, \eta\right) \geqslant$ $E_{\ell}^{r_{j}}\left(\left\{\underline{Q}_{r_{j}}^{(i)}\right\}_{i=1}^{I}, g_{r_{j}}\right)$. Similarly to 3.32, we conclude on noting 3.49a-c) and 2.16, that

$$
\liminf _{r_{j} \rightarrow 1} E_{\ell}^{r_{j}}\left(\left\{\underline{Q}_{r_{j}}^{(i)}\right\}_{i=1}^{I}, g_{r_{j}}\right) \geqslant \sum_{i=1}^{I} \int_{\bar{\Omega}}\left[\left|\underline{Q}^{(i)}\right|+\ell\left|\underline{\nabla} \cdot \underline{Q}^{(i)}-f^{(i)}+g\right|\right] .
$$

Similarly to 3.30, , we have

$$
\limsup _{r_{j} \rightarrow 1} E_{\ell}^{r_{j}}\left(\left\{\underline{\xi}^{(i)}\right\}_{i=1}^{I}, \eta\right) \leqslant \sum_{i=1}^{I} \int_{\bar{\Omega}}\left[\left|\underline{\xi}^{(i)}\right|+\ell\left|\underline{\nabla} \cdot \underline{\xi}^{(i)}-f^{(i)}+\eta\right|\right] .
$$

Hence, it follows on combining the above that $\underline{Q}^{(i)} \in \underline{V}_{0}^{\mathcal{M}}(\Omega), i=1 \rightarrow I$, and $g \in K_{G}(m)$ are such that

$$
\begin{aligned}
& \sum_{i=1}^{I} \int_{\bar{\Omega}}\left[\left|\underline{\xi}^{(i)}\right|+\ell\left|\underline{\nabla} \cdot \underline{\xi}^{(i)}-f^{(i)}+\eta\right|\right] \geqslant \sum_{i=1}^{I} \int_{\bar{\Omega}}\left[\left|\underline{Q}^{(i)}\right|+\ell\left|\underline{\nabla} \cdot \underline{Q}^{(i)}-f^{(i)}+g\right|\right] \\
& \forall \underline{\xi}^{(i)} \in\left[C_{0}^{\infty}(\Omega)\right]^{n}, i=1 \rightarrow I, \forall \eta \in K_{G}(m) .
\end{aligned}
$$




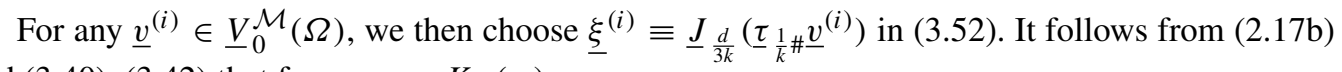
and $3.40-3.42$ that for any $\eta \in K_{G}(m)$,

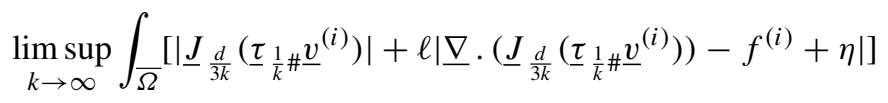

$$
\begin{aligned}
& \leqslant \int_{\bar{\Omega}}\left|\underline{v}^{(i)}\right|+\ell \limsup _{k \rightarrow \infty} \int_{\bar{\Omega}}\left[\left|\underline{\nabla} \cdot\left(\underline{J}_{\frac{d}{3 k}}\left(\underline{\tau}_{\frac{1}{k} \#} \underline{v}^{(i)}\right)\right)-J_{\frac{d}{3 k}}\left(\underline{\tau}_{\frac{1}{k} \#}\left(f^{(i)}-\eta\right)\right)\right|\right. \\
& \left.+\left|\left(f^{(i)}-\eta\right)-J_{\frac{d}{3 k}}\left(\tau_{\frac{1}{k} \#}\left(f^{(i)}-\eta\right)\right)\right|\right] \\
& \leqslant \int_{\bar{\Omega}}\left|\underline{v}^{(i)}\right|+\ell \limsup _{k \rightarrow \infty} \int_{\bar{\Omega}}\left|(1+1 / k)\left(\underline{\nabla} \cdot \underline{v}^{(i)}\right)-f^{(i)}+\eta\right| \\
& \leqslant \int_{\bar{\Omega}}\left[\left|\underline{v}^{(i)}\right|+\ell\left|\underline{\nabla} \cdot \underline{v}^{(i)}-f^{(i)}+\eta\right|\right] .
\end{aligned}
$$

Hence, there exists a solution to

$(\mathcal{P M})$ Find $\underline{Q}^{(i)} \in \underline{V}_{0}^{\mathcal{M}}(\Omega), i=1 \rightarrow I$, and $g \in K_{G}(m)$, such that

$$
\begin{gathered}
\int_{\bar{\Omega}}\left[|\underline{v}|+\ell\left|\underline{\nabla} \cdot \underline{v}-f^{(i)}+g\right|\right] \geqslant \int_{\bar{\Omega}}\left[\left|\underline{Q}^{(i)}\right|+\ell\left|\underline{\nabla} \cdot \underline{Q}^{(i)}-f^{(i)}+g\right|\right] \\
\forall \underline{v} \in \underline{V}_{0}^{\mathcal{M}}(\Omega), i=1 \rightarrow I, \\
\sum_{i=1}^{I} \int_{\bar{\Omega}}\left|\underline{\nabla} \cdot \underline{Q}^{(i)}-f^{(i)}+\eta\right| \geqslant \sum_{i=1}^{I} \int_{\bar{\Omega}}\left|\underline{\nabla} \cdot \underline{Q}^{(i)}-f^{(i)}+g\right| \quad \forall \eta \in K_{G}(m) ;
\end{gathered}
$$

that is, 2.11, with the restriction of $g$ to $\Omega_{g}$ and the upper bound constraint $G$ on $g$.

\section{Finite element approximation of $\left(\mathcal{P}_{r}\right),\left(\mathcal{U}_{r}\right)$ and $\left(\mathcal{P} \mathcal{M}_{r}\right)$}

For ease of exposition, we assume the following.

(A2) Let $n \leqslant 3$ with $\Omega, \Omega^{ \pm}$and $\Omega_{g}$ polytopes, if $n \geqslant 2$. Let $\left\{\mathcal{T}^{h}\right\}_{h>0}$ be a regular family of partitionings of $\Omega$ into disjoint open simplices $\sigma$ with $h_{\sigma}:=\operatorname{diam}(\sigma)$ and $h:=\max _{\sigma \in \mathcal{T}^{h}} h_{\sigma}$, so that

$$
\bar{\Omega}=\bigcup_{\sigma \in \mathcal{T}^{h}} \bar{\sigma}, \quad \text { with } \quad \overline{\Omega^{ \pm}}=\bigcup_{\sigma \in \mathcal{T}_{ \pm}^{h}} \bar{\sigma} \text { and } \quad \bar{\Omega}_{g}=\bigcup_{\sigma \in \mathcal{T}_{g}^{h}} \bar{\sigma}
$$

where $\mathcal{T}_{ \pm}^{h} \subset \mathcal{T}^{h}$ and $\mathcal{T}_{g}^{h} \subset \mathcal{T}^{h}$. We shall also assume that there exists $r_{0} \in(1,4 / 3]$, required for the proof of Lemma 4.1 below, such that for all $r \in\left(1, r_{0}\right)$,

$$
\Omega_{r}^{ \pm} \equiv \Omega^{ \pm}
$$

We note that the assumption 4.2 can always be achieved by defining $\Omega^{ \pm}$appropriately and using the general construction for $f_{r}^{ \pm}$as stated after $3.22 \mathrm{a}, \mathrm{b}$ ), because we require only that $\operatorname{supp}\left(f^{ \pm}\right) \subseteq \overline{\Omega^{ \pm}}$ 
We introduce the lowest order Raviart-Thomas finite element spaces

$$
\begin{aligned}
\underline{V}^{h} & :=\left\{\underline{v}^{h} \in\left[L^{\infty}(\Omega)\right]^{n}:\left.\underline{v}^{h}\right|_{\sigma}=\underline{a}_{\sigma}+b_{\sigma} \underline{x}, \underline{a}_{\sigma} \in \mathbb{R}^{n}, b_{\sigma} \in \mathbb{R} \forall \sigma \in \mathcal{T}^{h}\right\} \subset \underline{V}^{\infty}(\Omega), \\
S^{h} & :=\left\{\eta^{h} \in L^{\infty}(\Omega):\left.\eta^{h}\right|_{\sigma}=c_{\sigma} \in \mathbb{R} \forall \sigma \in \mathcal{T}^{h}\right\}, \\
S_{ \pm}^{h} & :=\left\{\eta^{h} \in L^{\infty}\left(\Omega^{ \pm}\right):\left.\eta^{h}\right|_{\sigma}=c_{\sigma} \in \mathbb{R} \forall \sigma \in \mathcal{T}_{ \pm}^{h}\right\}, \\
S_{g}^{h} & :=\left\{\eta^{h} \in L^{\infty}\left(\Omega_{g}\right):\left.\eta^{h}\right|_{\sigma}=c_{\sigma} \in \mathbb{R} \forall \sigma \in \mathcal{T}_{g}^{h}\right\}, \\
\underline{V}_{0}^{h} & :=\left\{\underline{v}^{h} \in \underline{V}^{h}: \underline{v}^{h} \cdot \underline{v}=0 \text { on } \partial \Omega\right\} \quad \text { and } \quad S_{M}^{h}:=\left\{\eta^{h} \in S^{h}:\left(\eta^{h}, 1\right)_{\Omega}=0\right\} .
\end{aligned}
$$

Here the constraint $\underline{V}^{h} \subset \underline{V}^{\infty}(\Omega)$ implies for all $\underline{v}^{h} \in \underline{V}^{h}$ and for all adjacent triangles $\sigma, \sigma^{\prime} \in \mathcal{T}^{h}$ that

$$
\left.\underline{v}^{h}\right|_{\sigma} \cdot \underline{v}_{\partial \sigma}+\left.\underline{v}^{h}\right|_{\sigma^{\prime}} \cdot \underline{v}_{\partial \sigma^{\prime}}=0 \quad \text { on } \partial \sigma \cap \partial \sigma^{\prime},
$$

where $\underline{v}_{\partial \sigma}$ is the outward unit normal to $\partial \sigma$, the boundary of $\sigma$.

In order for our finite element approximation to be practical, we introduce

$$
\begin{aligned}
(\underline{v}, \underline{z})^{h}:=\sum_{\sigma \in \mathcal{T}^{h}}(\underline{v}, \underline{z})_{\sigma}^{h} \quad \text { with } \quad(\underline{v}, \underline{z})_{\sigma}^{h}:=\frac{1}{n+1}|\sigma| \sum_{j=1}^{n+1} \underline{v}\left(P_{j}^{\sigma}\right) \cdot \underline{z}\left(P_{j}^{\sigma}\right) \\
\forall \underline{v}, \underline{z} \in[C(\bar{\sigma})]^{n}, \forall \sigma \in \mathcal{T}^{h},
\end{aligned}
$$

where $\left\{P_{j}^{\sigma}\right\}_{j=1}^{n+1}$ are the vertices of $\sigma$. Therefore $(\underline{v}, \underline{z})^{h}$ averages the integrand $\underline{v} \cdot \underline{z}$ over each simplex $\sigma$ at its vertices and hence is exact if $\underline{v}$. $z$ is piecewise linear over the partitioning $\mathcal{T}^{h}$. For any $r \geqslant 1$ and for any $\underline{v}^{h} \in \underline{V}^{h}$, we know from the equivalence of norms for $\underline{v}^{h}$ and the convexity of $|\cdot|^{r}$ that

$$
C_{r}\left(\left|\underline{v}^{h}\right|^{r}, 1\right)_{\sigma}^{h} \leqslant \int_{\sigma}\left|\underline{v}^{h}\right|^{r} \mathrm{~d} \underline{x} \leqslant\left(\left|\underline{v}^{h}\right|^{r}, 1\right)_{\sigma}^{h}:=\frac{1}{n+1}|\sigma| \sum_{j=1}^{n+1}\left|\underline{v}^{h}\left(P_{j}^{\sigma}\right)\right|^{r} \quad \forall \sigma \in \mathcal{T}^{h} .
$$

\subsection{Approximation of $\left(\mathcal{P}_{r}\right)$}

Our fully practical approximation of $\left(\mathcal{P}_{r}\right)$ for given $r \in\left(1, r_{0}\right)$ (recall 4.2 ), by $\underline{V}_{0}^{h}$ and $S^{h}$, on employing (4.5), is then:

$\left(\mathcal{P}_{r}^{h}\right)$ Find $\underline{Q}_{r}^{h} \in \underline{V}_{0}^{h}, q_{r}^{ \pm, h} \in S_{ \pm}^{h}, u_{r}^{h} \in S_{M}^{h}$ and $\lambda_{r}^{ \pm, h} \in \mathbb{R}$ such that

$$
\begin{array}{ll}
\left(\left|\underline{Q}_{r}^{h}\right|^{r-2} \underline{Q}_{r}^{h}, \underline{v}^{h}\right)^{h}=\left(u_{r}^{h}, \underline{\nabla} \cdot \underline{v}^{h}\right)_{\Omega} & \forall \underline{v}^{h} \in \underline{V}_{0}^{h}, \\
\ell\left(\left|q_{r}^{+, h}\right|^{r-2} q_{r}^{+, h}, v^{+, h}\right)_{\Omega^{+}}=\left(u_{r}^{h}-\lambda_{r}^{+, h}, v^{+, h}\right)_{\Omega^{+}} & \forall v^{+, h} \in S_{+}^{h}, \\
\ell\left(\left|q_{r}^{-, h}\right|^{r-2} q_{r}^{-, h}, v^{-, h}\right)_{\Omega^{-}}=-\left(u_{r}^{h}+\lambda_{r}^{-, h}, v^{-, h}\right)_{\Omega^{-}} & \forall v^{-, h} \in S_{-}^{h}, \\
\left(\underline{\nabla} \cdot \underline{Q}_{r}^{h}, \eta^{h}\right)_{\Omega}=\left(f_{r}^{+}-q_{r}^{+, h}, \eta^{h}\right)_{\Omega^{+}}-\left(f_{r}^{-}-q_{r}^{-, h}, \eta^{h}\right)_{\Omega^{-}} & \forall \eta^{h} \in S^{h}, \\
\left(f_{r}^{+}-q_{r}^{+, h}, 1\right)_{\Omega^{+}}=\left(f_{r}^{-}-q_{r}^{-, h}, 1\right)_{\Omega^{-}}=m . &
\end{array}
$$

Let $P_{( \pm)}^{h}: L^{1}\left(\Omega^{( \pm)}\right) \rightarrow S_{( \pm)}^{h}$ be such that

$$
\left(\left(I-P_{( \pm)}^{h}\right) z, \eta^{h}\right)_{\Omega^{( \pm)}}=0 \quad \forall \eta^{h} \in S_{( \pm)}^{h},
$$


where $( \pm)$ means with or without these subscripts and superscripts. It follows that for all $z \in$ $L^{s}\left(\Omega^{( \pm)}\right)$, where $s \in[1, \infty]$,

$$
\left|P_{( \pm)}^{h} z\right|_{0, s, \Omega^{( \pm)}} \leqslant|z|_{0, s, \Omega^{( \pm)}} \quad \text { and } \quad \lim _{h \rightarrow 0}\left|\left(I-P_{( \pm)}^{h}\right) z\right|_{0, s, \Omega^{( \pm)}}=0 .
$$

Then, similarly to 3.4 and 3.5 , we introduce, for $\rho \in L_{M}^{1}(\Omega)$,

$$
\underline{X}^{h}(\rho):=\left\{\underline{v}^{h} \in \underline{V}_{0}^{h}:\left(\underline{\nabla} \cdot \underline{v}^{h}, \eta^{h}\right)_{\Omega}=\left(\rho, \eta^{h}\right)_{\Omega} \forall \eta^{h} \in S^{h}\right\},
$$

and

$$
\begin{array}{r}
\underline{Y}^{h}\left(f_{r}^{+}, f_{r}^{-}, m\right):=\left\{\left(\underline{v}^{h}, v^{+, h}, v^{-, h}\right) \in \underline{X}^{h}\left(P^{h}\left(\left[f_{r}^{+}-v^{+, h}\right]-\left[f_{r}^{-}-v^{-, h}\right]\right)\right) \times S_{+}^{h} \times S_{-}^{h}:\right. \\
\left.\left(f_{r}^{+}-v^{+, h}, 1\right)_{\Omega^{+}}=\left(f_{r}^{-}-v^{-, h}, 1\right)_{\Omega^{-}}=m\right\} .
\end{array}
$$

It follows from 3.1, 4.10 and 4.11 that a solution of $\left(\mathcal{P}_{r}^{h}\right)$ is such that $\left(Q_{r}^{h}, q_{r}^{+, h}, q_{r}^{-, h}\right) \in$ $\underline{Y}^{h}\left(f_{r}^{+}, f_{r}^{-}, m\right)$ and

$$
\begin{array}{r}
E_{\ell}^{r, h}\left(\underline{Q}_{r}^{h}, q_{r}^{+, h}, q_{r}^{-, h}\right) \leqslant E_{\ell}^{r, h}\left(\underline{v}^{h}, v^{+, h}, v^{-, h}\right):=\frac{1}{r}\left[\left(\left|\underline{v}^{h}\right|^{r}, 1\right)^{h}+\ell\left(\left|v^{+, h}\right|^{r}, 1\right)_{\Omega^{+}}+\ell\left(\left|v^{-, h}\right|^{r}, 1\right)_{\Omega^{-}}\right] \\
\forall\left(\underline{v}^{h}, v^{+, h}, v^{-, h}\right) \in \underline{Y}^{h}\left(f_{r}^{+}, f_{r}^{-}, m\right) .
\end{array}
$$

For $r>1$, let $\underline{I}^{h}: \underline{V}_{0}^{r}(\Omega) \cap\left[W^{1, r}(\Omega)\right]^{n} \rightarrow \underline{V}_{0}^{h}$ be the generalised interpolation operator satisfying

$$
\int_{\partial_{i} \sigma}\left(\underline{v}-\underline{I}^{h} \underline{v}\right) \cdot \underline{v}_{\partial_{i} \sigma} \mathrm{d} s=0, \quad i=1 \rightarrow n+1, \forall \sigma \in \mathcal{T}^{h},
$$

where $\partial \sigma \equiv \bigcup_{i=1}^{n+1} \partial_{i} \sigma$ and $\underline{v}_{\partial_{i} \sigma}$ are the corresponding outward unit normals on $\partial_{i} \sigma$. It follows that

$$
\left(\underline{\nabla} \cdot\left(\underline{v}-\underline{I}^{h} \underline{v}\right), \eta^{h}\right)=0 \quad \forall \eta^{h} \in S^{h} .
$$

In addition, for all $\sigma \in \mathcal{T}^{h}$ and any $s \in(1, \infty]$ we have

$$
\underline{\mid v}-\left.\underline{I}^{h} \underline{v}\right|_{0, s, \sigma} \leqslant C_{s} h_{\sigma}|\underline{v}|_{1, s, \sigma} \quad \text { and } \quad\left|\underline{I}^{h} \underline{v}\right|_{1, s, \sigma} \leqslant C_{s}|\underline{v}|_{1, s, \sigma}
$$

(see e.g. [15, Lemma 3.1]; the proof given there for $s \geqslant 2$ is also valid for any $s \in(1, \infty])$; and, if $\underline{v}$ is sufficiently smooth,

$$
\left|\underline{\nabla} \cdot\left(\underline{v}-\underline{I}^{h} \underline{v}\right)\right|_{0, \sigma} \leqslant C h|\underline{\nabla} \cdot \underline{v}|_{1, \sigma}
$$

(see e.g. [20, p. 553]). Furthermore, we note from 3.1, , 4.5) and 4.15a) that for all $\sigma \in \mathcal{T}^{h}$ and $\underline{v} \in\left[W^{1, \infty}(\sigma)\right]^{n}$,

$$
\begin{aligned}
\left.\left|\int_{\sigma}\right| \underline{v}\right|^{r} \mathrm{~d} \underline{x}-\left(\left|\underline{I}^{h} \underline{v}\right|^{r}, 1\right)_{\sigma}^{h} \mid & \leqslant\left.\int_{\sigma}|| \underline{v}\right|^{r}-\left|\underline{I^{h}} \underline{v}\right|^{r}|\mathrm{~d} \underline{x}+| \int_{\sigma}\left|\underline{I}^{h} \underline{v}\right|^{r} \mathrm{~d} \underline{x}-\left(\left|\underline{I}^{h} \underline{v}\right|^{r}, 1\right)_{\sigma}^{h} \mid \\
& \leqslant r\left(|\underline{v}|_{0, \infty, \sigma}^{r-1}+\left|\underline{I}^{h} \underline{v}\right|_{0, \infty, \sigma}^{r-1}\right)\left[\left|\underline{v}-\underline{I}^{h} \underline{v}\right|_{0,1, \sigma}+h\left|\underline{I}^{h} \underline{v}\right|_{1,1, \sigma}\right] \\
& \leqslant C_{r} h\|\underline{v}\|_{1, \infty, \sigma}^{r} .
\end{aligned}
$$

We have the following discrete version of Lemma 3.1 . 
Lemma 4.1 Let the Assumptions (A1) and (A2) hold. Then for all $r \in\left(1, r_{0}\right)$ and $p=r /(r-1)$, given $\rho^{h} \in S_{M}^{h}$ there exists $\underline{Q}_{r}^{\rho^{h}, h} \in \underline{X}^{h}\left(\rho^{h}\right)$ and

$$
\left\|\underline{Q}_{r}^{\rho^{h}, h}\right\|_{\underline{V}^{r}(\Omega)} \leqslant \mu_{r}\left|\rho^{h}\right|_{0, r, \Omega},
$$

where $\mu_{r} \in \mathbb{R}_{>0}$ is independent of $h$, but possibly dependent on $r$. Hence it follows that

$$
\inf _{\eta^{h} \in S_{M}^{h}} \sup _{\underline{v}^{h} \in \underline{V}_{0}^{h}} \frac{\left(\underline{\nabla} \cdot \underline{v}^{h}, \eta^{h}\right)}{\left\|\underline{v}^{h}\right\|_{\underline{V}^{r}(\Omega)}\left|\eta^{h}\right|_{0, p, \Omega}} \geqslant\left[2 \mu_{r}\right]^{-1} .
$$

Proof. See the proof of Lemma 3.1 in [5].

THEOREM 4.1 Let the Assumptions (A1) and (A2) hold. Then for any given $r \in\left(1, r_{0}\right)$ there exists a unique solution, $\left(Q_{r}^{h}, q_{r}^{+, h}, q_{r}^{-, h}, u_{r}^{h}, \lambda_{r}^{+, h}, \lambda_{r}^{-, h}\right) \in \underline{V}_{0}^{h} \times S_{+}^{h} \times S_{-}^{h} \times S_{M}^{h} \times \mathbb{R} \times \mathbb{R}$, to $\left(\mathcal{P}_{r}^{h}\right)$. In addition,

$$
\begin{array}{r}
\left\|\underline{Q}_{r}^{h}\right\|_{\underline{\underline{r}}^{r}(\Omega)}^{r}+\ell\left|q_{r}^{+, h}\right|_{0_{0, r} \Omega^{+}}^{r}+\ell\left|q_{r}^{-, h}\right|_{0, r, \Omega^{-}}^{r} \leqslant C_{\Omega, \ell, r}\left[\left|f_{r}^{+}\right|_{0, r, \Omega^{+}}^{r}+\left|f_{r}^{-}\right|_{0, r, \Omega^{-}}^{r}\right], \\
\left|u_{r}^{h}\right|_{0, p, \Omega}+\left|\Omega^{+}\right|^{(r-1) / r}\left|\lambda_{r}^{+, h}\right|+\left|\Omega^{-}\right|^{(r-1) / r}\left|\lambda_{r}^{-, h}\right| \leqslant C_{\Omega, \ell, r}\left[\left|f_{r}^{+}\right|_{0, r, \Omega^{+}}^{r-1}+\left|f_{r}^{-}\right|_{0, r, \Omega^{-}}^{r-1}\right],
\end{array}
$$

where $p=r /(r-1)$.

Proof. The proof is a discrete analogue of the proof of Theorem 3.1 First, we define $\rho:=$ $\left[f_{r}^{+}-q_{r}^{f,+}\right]-\left[f_{r}^{-}-q_{r}^{f,-}\right] \in L_{M}^{r}(\Omega)$, where $q_{r}^{f, \pm}$ are defined by 3.10. It follows from 4.17, 4.9 and 3.11 that there exists $\underline{Q}_{r}^{\rho, h} \in \underline{X}^{r}\left(P^{h} \rho\right)$ and

$$
\left\|\underline{Q}_{r}^{\rho, h}\right\|_{\underline{V}^{r}(\Omega)} \leqslant C_{\Omega, r}\left|P^{h} \rho\right|_{0, r, \Omega} \leqslant C_{\Omega, r}|\rho|_{0, r, \Omega} \leqslant C_{\Omega, r}\left[\left|f_{r}^{+}\right|_{0, r, \Omega^{+}}+\left|f_{r}^{-}\right|_{0, r, \Omega^{-}}\right] .
$$

Therefore, on setting $\underline{Q}_{r}^{h}:=\underline{Q}_{r}^{h}-\underline{Q}_{r}^{\rho, h}$ and $\widehat{q}_{r}^{ \pm, h}:=q_{r}^{ \pm, h}-P_{ \pm}^{h} q_{r}^{f, \pm}$, the problem $\left.\left(\mathcal{P}_{r}^{h}\right), 4.7 \mathrm{a}-\mathrm{e}\right)$, can be reduced to: Find $\left(\underline{\underline{Q}}_{r}^{h}, \widehat{q}_{r}^{+, h}, \widehat{q}_{r}^{-, h}\right) \in \underline{Y}^{h}(0,0,0)$ such that

$$
\begin{aligned}
& \left(\left|\underline{\widehat{Q}}_{r}^{h}+\underline{Q}_{r}^{\rho, h}\right|^{r-2}\left(\widehat{\widehat{Q}}_{r}^{h}+\underline{Q}_{r}^{\rho, h}\right), \underline{v}^{h}\right)^{h}+\ell\left(\left|\widehat{q}_{r}^{,, h}+P_{+}^{h} q_{r}^{f,+}\right|^{r-2}\left(\widehat{q}_{r}^{+, h}+P_{+}^{h} q_{r}^{f,+}\right), v^{+, h}\right)_{\Omega^{+}} \\
& \quad+\ell\left(\left|\widehat{q}_{r}^{,, h}+P_{-}^{h} q_{r}^{f,-}\right|^{r-2}\left(\widehat{q}_{r}^{, h}+P_{-}^{h} q_{r}^{f,-}\right), v^{-, h}\right)_{\Omega^{-}}=0 \quad \forall\left(\underline{v}^{h}, v^{+, h}, v^{-, h}\right) \in \underline{Y}^{h}(0,0,0),
\end{aligned}
$$

which is the Euler-Lagrange equation for the strictly convex minimization problem

$$
\inf _{\left(\underline{v}^{h}, v^{+, h}, v^{-, h}\right) \in \underline{Y}^{h}(0,0,0)} E_{\ell}^{r, h}\left(\underline{v}^{h}+\underline{Q}_{r}^{\rho, h}, v^{+, h}+P_{+}^{h} q_{r}^{f,+}, v^{-, h}+P_{-}^{h} q_{r}^{f,-}\right) .
$$

Hence there exists a unique solution $\left(\widehat{Q}_{r}^{h}, \widehat{q}_{r}^{+, h}, \widehat{q}_{r}^{-, h}\right) \in \underline{Y}^{h}(0,0,0)$ to 4.21 . On setting $Q_{r}^{h}=\widehat{Q}_{r}^{h}+Q_{r}^{\rho, h}$ and $q_{r}^{ \pm, h}=\widehat{q}_{r}^{ \pm, h}+P_{ \pm}^{h} q_{r}^{f, \pm}$, it follows from 4.21 that $\left(\underline{Q}_{r}^{h}, q_{r}^{+, h}, q_{r}^{-, h}\right) \in$ $\underline{Y}^{h}\left(f_{r}^{+}, f_{r}^{-}, m\right)$ is such that

$$
\begin{array}{r}
\left(\left|\underline{Q}_{r}^{h}\right|^{r-2} \underline{Q}_{r}^{h}, \underline{v}^{h}\right)^{h}+\ell\left(\left|q_{r}^{+, h}\right|^{r-2} q_{r}^{+, h}, v^{+, h}\right)_{\Omega^{+}}+\ell\left(\left|q_{r}^{-, h}\right|^{r-2} q_{r}^{-, h}, v^{-, h}\right)_{\Omega^{-}}=0 \\
\forall\left(\underline{v}^{h}, v^{+, h}, v^{-, h}\right) \in \underline{Y}^{h}(0,0,0),
\end{array}
$$


and hence, in particular with $v^{ \pm, h} \equiv 0$, that

$$
\left(\left|\underline{Q}_{r}^{h}\right|^{r-2} \underline{Q}_{r}^{h}, \underline{v}^{h}\right)^{h}=0 \quad \forall \underline{v}^{h} \in \underline{X}^{r, h}(0) .
$$

It is also easily deduced from $4.7 \mathrm{a}-\mathrm{e})$ and 4.11 that any solution $\left(Q_{r}^{h}, q_{r}^{ \pm, h}\right)$ of $\left(\mathcal{P}_{r}^{h}\right)$ solves (4.23a), and from (3.2) that it is unique. In addition, it follows from (4.22), 4.6, (4.9) and 4.11) that

$$
\begin{array}{r}
\left|\underline{Q}_{r}^{h}\right|_{0, r, \Omega}^{r}+\left|q_{r}^{+, h}\right|_{0, r, \Omega^{+}}^{r}+\left|q_{r}^{-, h}\right|_{0, r, \Omega^{-}}^{r} \leqslant C_{\Omega, \ell, r}\left[\left|\underline{Q}_{r}^{\rho, h}\right|_{0, r, \Omega}^{r}+\left|q_{r}^{f,+}\right|_{0, r, \Omega^{+}}^{r}+\left|q_{r}^{f,-}\right|_{0, r, \Omega^{-}}^{r}\right], \\
\left|\underline{\nabla} \cdot \underline{Q}_{r}^{h}\right|_{0, r, \Omega}^{r} \leqslant\left[\left|f_{r}^{+}-q_{r}^{+, h}\right|_{0, r, \Omega^{+}}+\left|f_{r}^{-}-q_{r}^{-, h}\right|_{0, r, \Omega^{-}}\right]^{r} .
\end{array}
$$

The bound 4.19a follows immediately from 4.24a b), 4.20) and (3.11).

Similarly to the proof of Theorem 3.1 , we show that there exists a unique $u_{r}^{h} \in S_{M}^{h}$ satisfying 4.7a). In addition, from 4.18, 4.7a) and 4.6 we have

$$
\left|u_{r}^{h}\right|_{0, p, \Omega} \leqslant 2 \mu_{r} \sup _{\underline{v}^{h} \in \underline{V}_{0}^{r}(\Omega)} \frac{\left(\left|\underline{Q}_{r}^{h}\right|^{r-2} \underline{Q}_{r}^{h}, \underline{v}^{h}\right)^{h}}{\left\|\underline{v}^{h}\right\|_{\underline{V}^{r}(\Omega)}} \leqslant C_{r}\left|\underline{Q}_{r}^{h}\right|_{0, r, \Omega}^{r-1} .
$$

Choosing $v^{\mp, h} \equiv 0$ in $4.23 \mathrm{a}$, we see that $v^{ \pm, h}=\mp \underline{\nabla} \cdot \underline{v}^{h}$; and hence, on noting $4.7 \mathrm{a}$, that $\ell\left|q_{r}^{ \pm, h}\right|^{r-2} q_{r}^{ \pm, h} \mp u_{r}^{h}$ are constant on $\Omega^{ \pm}$. On choosing

$$
\lambda_{r}^{ \pm, h}=-\ell\left|q_{r}^{ \pm, h}\right|^{r-2} q_{r}^{ \pm, h} \pm u_{r}^{h},
$$

we deduce that $4.7 \mathrm{~b}$ c) hold for general $v^{ \pm, h} \in S_{ \pm}^{h}$. Therefore, we have proved that there exists a unique solution to $\left.\left(\mathcal{P}_{r}^{h}\right), 4.7 \mathrm{a}-\mathrm{e}\right)$, which is equivalent to the minimization problem 4.12. Furthermore, we infer from 4.26 that

$$
\left|\lambda_{r}^{ \pm, h}\right| \leqslant\left|\Omega^{ \pm}\right|^{-\frac{r-1}{r}}\left[\ell\left|q_{r}^{ \pm, h}\right|_{0, r, \Omega^{ \pm}}^{r-1}+\left|u_{r}^{h}\right|_{0, p, \Omega}\right] .
$$

Combining 4.25, 4.19a and 4.27) yields the desired result 4.19b).

THEOREM 4.2 Let the Assumptions (A1) and (A2) hold. For all $r \in\left(1, r_{0}\right)$, the unique solution $\left(\underline{Q}_{r}^{h}, q_{r}^{+, h}, q_{r}^{-, h}, u_{r}^{h}, \lambda_{r}^{+, h}, \lambda_{r}^{-, h}\right)$ of $\left(\mathcal{P}_{r}^{h}\right)$ is such that as $h \rightarrow 0$,

$$
\begin{aligned}
\underline{Q}_{r}^{h} & \rightarrow \underline{Q}_{r} & & \text { weakly in }\left[L^{r}(\Omega)\right]^{n}, \\
\underline{\nabla} \cdot \underline{Q}_{r}^{h} & \rightarrow \underline{\nabla} \cdot \underline{Q}_{r} & & \text { weakly in } L^{r}(\Omega), \\
q_{r}^{ \pm, h} & \rightarrow q_{r}^{ \pm} & & \text {weakly in } L^{r}\left(\Omega^{ \pm}\right), \\
u_{r}^{h} & \rightarrow u_{r} & & \text { weakly in } L^{p}(\Omega), \\
\lambda_{r}^{ \pm, h} & \rightarrow \lambda_{r}^{ \pm}, & &
\end{aligned}
$$

where $\left(\underline{Q}_{r}, q_{r}^{+}, q_{r}^{-}, u_{r}, \lambda_{r}^{+}, \lambda_{r}^{-}\right)$is the unique solution of $\left(\mathcal{P}_{r}\right)$.

Proof. The results $4.28 \mathrm{a}-\mathrm{e})$ follow immediately for a subsequence $\left\{\left(\underline{Q}_{r}^{h_{j}}, q_{r}^{ \pm, h_{j}}, u_{r}^{h_{j}}, \lambda_{r}^{ \pm, h_{j}}\right)\right\}_{h_{j}>0}$ of $\left\{\left(\underline{Q}_{r}^{h}, q_{r}^{ \pm, h}, u_{r}^{h}, \lambda_{r}^{ \pm, h}\right)\right\}_{h>0}$ from $4.19 \mathrm{a}$ b). 
It follows from (4.28c that we may pass to the limit in the $h_{j}$ version of (4.7e to obtain $3.3 \mathrm{e}$, on recalling 4.2). For any $\eta \in L^{p}(\Omega)$, we choose $\eta^{h}=P^{h} \eta \in S^{h}$ in the $h_{j}$ version of (4.7d). Noting 4.28b c), and (4.9), we obtain the desired result (3.3d).

For any $\underline{\xi} \in\left[C_{0}^{\infty}(\Omega)\right]^{n}$ and $\xi^{ \pm} \in C_{0}^{\infty}(\Omega)$, we choose $\underline{v}^{h}=\underline{I}^{h_{j}} \underline{\xi}-\underline{Q}_{r}^{h_{j}}$ and $v^{ \pm, h}=P_{ \pm}^{h^{ \pm}} \xi^{ \pm}$in the $h_{j}$ versions of $\left.4.7 \mathrm{a}-\mathrm{c}\right)$. Hence, on noting (3.1), we have

$$
\begin{aligned}
& \left(u_{r}^{h_{j}}, \underline{\nabla} \cdot\left(\underline{Q}_{r}^{h_{j}}-\underline{I}^{h_{j}} \underline{\xi}\right)\right)_{\Omega}=\left(\left|\underline{Q}_{r}^{h_{j}}\right|^{r-2} \underline{Q}_{r}^{h_{j}}, \underline{Q}_{r}^{h_{j}}-\underline{I}^{h_{j}} \underline{\xi}\right)^{h_{j}} \\
& \geqslant \frac{1}{r}\left(\left|\underline{Q}_{r}^{h_{j}}\right|^{r}-\left|\underline{I}^{h_{j}} \underline{\xi}\right|^{r}, 1\right)^{h_{j}}, \\
& \left(u_{r}^{h_{j}}-\lambda_{r}^{+, h_{j}}, q_{r}^{+, h_{j}}-P_{+}^{h_{j}} \xi^{+}\right)_{\Omega^{+}}=\ell\left(\left|q_{r}^{+, h_{j}}\right|^{r-2} q_{r}^{+, h_{j}}, q_{r}^{+, h_{j}}-P_{+}^{h_{j}} \xi^{+}\right)_{\Omega^{+}} \\
& \geqslant \frac{\ell}{r}\left(\left|q_{r}^{+, h_{j}}\right|^{r}-\left|P_{+}^{h_{j}} \xi^{+}\right|^{r}, 1\right)_{\Omega^{+}}, \\
& -\left(u_{r}^{h_{j}}+\lambda_{r}^{-, h_{j}}, q_{r}^{-, h_{j}}-P_{-}^{h_{j}} \xi^{-}\right)_{\Omega^{-}}=\ell\left(\left|q_{r}^{-, h_{j}}\right|^{r-2} q_{r}^{-, h_{j}}, q_{r}^{-, h_{j}}-P_{-}^{h_{j}} \xi^{-}\right)_{\Omega^{-}} \\
& \geqslant \frac{\ell}{r}\left(\left|q_{r}^{-, h_{j}}\right|^{r}-\left|P_{-}^{h_{j}} \xi^{-}\right|^{r}, 1\right)_{\Omega^{-}} .
\end{aligned}
$$

Summing the above, and noting 4.7d and 4.14, yields

$$
\begin{gathered}
-\left(u_{r}^{h_{j}}, \underline{\nabla} \cdot \underline{\xi}\right)_{\Omega}+\left(f_{r}^{+}-P_{+}^{h_{j}} \xi^{+}, u_{r}^{h_{j}}\right)_{\Omega^{+}}-\left(f_{r}^{-}-P_{-}^{h_{j}} \xi^{-}, u_{r}^{h_{j}}\right)_{\Omega^{-}} \\
-\left(\lambda_{r}^{+, h_{j}}, q_{r}^{+, h_{j}}-P_{+}^{h_{j}} \xi^{+}\right)_{\Omega^{+}}-\left(\lambda_{r}^{-, h_{j}}, q_{r}^{-, h_{j}}-P_{-}^{h_{j}} \xi^{-}\right)_{\Omega^{-}} \\
=\left(u_{r}^{h_{j}}, \underline{\nabla} \cdot\left(\underline{Q}_{r}^{h_{j}}-\underline{I}^{h_{j}} \underline{\xi}\right)\right)_{\Omega}+\left(u_{r}^{h_{j}}-\lambda_{r}^{+, h_{j}}, q_{r}^{+, h_{j}}-P_{+}^{h_{j}} \xi^{+}\right)_{\Omega^{+}} \\
-\left(u_{r}^{h_{j}}+\lambda_{r}^{-, h_{j}}, q_{r}^{-, h_{j}}-P_{-}^{h_{j}} \xi^{-}\right)_{\Omega^{-}} \\
\geqslant \frac{1}{r}\left[\left(\left|\underline{Q_{r}^{h_{j}}}\right|^{r}-\left|\underline{I}^{h_{j}} \underline{\xi}\right|^{r}, 1\right)^{h_{j}}+\ell\left(\left|q_{r}^{+, h_{j}}\right|^{r}-\left|P_{+}^{h_{j}} \xi^{+}\right|^{r}, 1\right)_{\Omega^{+}}\right. \\
\left.+\ell\left(\left|q_{r}^{-, h_{j}}\right|^{r}-\left|P_{-}^{h_{j}} \xi^{-}\right|^{r}, 1\right)_{\Omega^{-}}\right] .
\end{gathered}
$$

We conclude from (4.6, 3.1) and 4.28a,c) that

$$
\liminf _{h_{j} \rightarrow 0}\left(\left|\underline{Q}_{r}^{h_{j}}\right|^{r}, 1\right)^{h_{j}} \geqslant\left(\left|\underline{Q_{r}}\right|^{r}, 1\right)_{\Omega} \quad \text { and } \quad \liminf _{h_{j} \rightarrow 0}\left(\left|\underline{q}_{r}^{ \pm, h_{j}}\right|^{r}, 1\right)_{\Omega^{ \pm}} \geqslant\left(\left|\underline{q}_{r}^{ \pm}\right|^{r}, 1\right)_{\Omega^{ \pm}}
$$

Passing to the limit $h_{j} \rightarrow 0$ in 4.30, and noting (4.28a-e), 4.9, 4.16) and (4.31), yields

$$
\begin{gathered}
-\left(u_{r}, \underline{\nabla} \cdot \underline{\xi}\right)_{\Omega}+\left(f_{r}^{+}-\xi^{+}, u_{r}\right)_{\Omega^{+}}-\left(f_{r}^{-}-\xi^{-}, u_{r}\right)_{\Omega^{-}}-\left(\lambda_{r}^{+}, q_{r}^{+}-\xi^{+}\right)_{\Omega^{+}}-\left(\lambda_{r}^{-}, q_{r}^{-}-\xi^{-}\right)_{\Omega^{-}} \\
\geqslant \frac{1}{r}\left[\left(\left|\underline{Q_{r}}\right|^{r}-|\underline{\xi}|^{r}, 1\right)_{\Omega}+\ell\left(\left|q_{r}^{+}\right|^{r}-\left|\xi^{+}\right|^{r}, 1\right)_{\Omega^{+}}+\ell\left(\left|q_{r}^{-}\right|^{r}-\left|\xi^{-}\right|^{r}, 1\right)_{\Omega^{-}}\right] \\
\forall\left(\underline{\xi}, \xi^{+}, \xi^{-}\right) \in\left[C_{0}^{\infty}(\Omega)\right]^{n} \times C_{0}^{\infty}\left(\Omega^{+}\right) \times C_{0}^{\infty}\left(\Omega^{-}\right) .
\end{gathered}
$$

Recalling that $\underline{V}_{0}^{r}(\Omega)$ and $L^{r}\left(\Omega^{ \pm}\right)$are the strong closures of $\left[C_{0}^{\infty}(\Omega)\right]^{n}$ and $C_{0}^{\infty}\left(\Omega^{ \pm}\right)$in the norms $\|\cdot\|_{\underline{V}^{r}(\Omega)}$ and $|\cdot|_{0, r, \Omega^{ \pm}}$, respectively, we find that 4.32 remains true for all $\left(\underline{\xi}, \xi^{+}, \xi^{-}\right) \in \underline{V}_{0}^{r}(\Omega) \times$ $L^{r}\left(\Omega^{+}\right) \times L^{r}\left(\Omega^{-}\right)$.

Therefore choosing $\underline{\xi}=Q_{r} \pm \varepsilon \underline{v}$ and $\xi^{ \pm}=q_{r}^{ \pm}$with $\underline{v} \in \underline{V}_{0}^{r}(\Omega)$ in 4.32 , and noting (3.1) and 3.3d, yields the desired result $3.3 \mathrm{a}$, on letting $\varepsilon \rightarrow 0$. Similarly, choosing $\underline{\xi}=\underline{Q}_{r}$ and $\xi^{ \pm}=q_{r}^{ \pm} \pm$ 
$\varepsilon v^{ \pm}$with $v^{ \pm} \in L^{r}\left(\Omega^{ \pm}\right)$in 4.32 , and noting 3.1 and $\sqrt{3.3 \mathrm{~d}}$, yields the desired results $3.3 \mathrm{~b}$ c), on letting $\varepsilon \rightarrow 0$. Hence $\left(\underline{Q}_{r}, q_{r}^{+}, q_{r}^{-}, u_{r}, \lambda^{+}, \lambda^{-}\right) \in \underline{V}_{0}^{r}(\Omega) \times L^{r}\left(\Omega^{+}\right) \times L^{r}\left(\Omega^{-}\right) \times L^{p}(\Omega) \times$ $\mathbb{R} \times \mathbb{R}$ solves $\left(\mathcal{P}_{r}\right)$. As the solution of $\left(\mathcal{P}_{r}\right)$ is unique, the whole sequence $\left\{\left(\underline{Q}_{r}^{h}, q_{r}^{ \pm, h}, u_{r}^{h}, \lambda_{r}^{ \pm}, h\right)\right\}_{h>0}$ converges in $(4.28 \mathrm{a}-\mathrm{e})$.

REMARK 4.1 Clearly, on combining Theorems 3.2 and 4.2 we have the subsequence convergence of $\left\{\left(\underline{Q}_{r}^{h}, q_{r}^{+, h}, q_{r}^{-, h}, u_{r}^{h}, \lambda_{r}^{+, h}, \lambda_{r}^{-, h}\right)\right\}_{(r>1, h>0)}$ as $r_{j} \rightarrow 1$ and $h_{j} \rightarrow 0$ to $\left(\underline{Q}, q^{+}, q^{-}, u, \lambda^{+}, \lambda^{-}\right) \in$ $\underline{V}_{0}^{\mathcal{M}}(\Omega) \times \mathcal{M}\left(\overline{\Omega^{+}}\right) \times \mathcal{M}\left(\overline{\Omega^{-}}\right) \times C_{M}(\bar{\Omega}) \times \mathbb{R} \times \mathbb{R}$, which is a solution of $\left.(\widehat{\mathcal{P}}), 3.23 \mathrm{a}-\mathrm{e}\right)$. In the absence of a uniqueness result for $(\widehat{\mathcal{P}})$, we have only subsequence convergence here. We note that for balanced $L^{1}$ MK problems with no free Dirichlet sets, the uniqueness of the optimal transportation flux $q$ was proved under the assumption of convexity of $\Omega$ and sufficient regularity of $f^{ \pm}$(see [16]). Under similar assumptions, for problems with a free Dirichlet set, the uniqueness of the transport density $a=|q|$ outside of this set was shown in [9].

\subsection{Approximation of $\left(\mathcal{U}_{r}\right)$}

Similarly to subsection 3.2, we assume that $f:=f^{+}-f^{-} \in L^{r_{0}}(\Omega)$ for some $r_{0}>1$. Then for a given $r \in\left(1, r_{0}\right)$, we consider the following fully practical approximation of $\left(\mathcal{U}_{r}\right)$ by $\underline{V}_{0}^{h}$ :

$\left(\mathcal{U}_{r}^{h}\right)$ Find $\underline{Q}_{r}^{h} \in \underline{V}_{0}^{h}$ such that

$$
\left(\left|\underline{Q}_{r}^{h}\right|^{r-2} \underline{Q}_{r}^{h}, \underline{v}^{h}\right)^{h}+\ell\left(\left|\underline{\nabla} \cdot \underline{Q}_{r}^{h}-P^{h} f\right|^{r-2}\left(\underline{\nabla} \cdot \underline{Q}_{r}^{h}-P^{h} f\right), \underline{\nabla} \cdot \underline{v}^{h}\right)_{\Omega}=0 \quad \forall \underline{v}^{h} \in \underline{V}_{0}^{h} .
$$

$\left(\mathcal{U}_{r}^{h}\right)$ is the Euler-Lagrange equation for the following strictly convex minimization problem: Find $\underline{Q}_{r}^{h} \in \underline{V}_{0}^{h}$ such that

$$
E_{\ell}^{r, h}\left(\underline{Q}_{r}^{h}\right) \leqslant E_{\ell}^{r, h}\left(\underline{v}^{h}\right):=\frac{1}{r}\left[\left(\left|\underline{v}^{h}\right|^{r}, 1\right)^{h}+\ell\left(\left|\underline{\nabla} \cdot \underline{v}^{h}-P^{h} f\right|^{r}, 1\right)_{\Omega}\right] \quad \forall \underline{v}^{h} \in \underline{V}_{0}^{h} .
$$

Hence, there exists a unique solution $\underline{Q}_{r}^{h} \in \underline{V}_{0}^{h}$ to $\left(\mathcal{U}_{r}^{h}\right)$; and moreover, on noting 4.6 and 4.9 , we have

$$
\left|\underline{Q}_{r}^{h}\right|_{0, r, \Omega}^{r}+\ell\left|\underline{\nabla} \cdot \underline{Q}_{r}^{h}-P^{h} f\right|_{0, r, \Omega}^{r} \leqslant C_{\ell, r}|f|_{0, r, \Omega}^{r} .
$$

It follows from 3.36 that there exists $\underline{Q}_{r} \in \underline{V}_{0}^{r}(\Omega)$ such that there exists a subsequence $\left\{\underline{Q}_{r}^{h_{j}}\right\}_{h_{j}>0}$, where $\underline{Q}_{r}^{h}$ is the unique solution of $\left(\mathcal{U}_{r}^{h}\right)$, such that $4.28 \mathrm{a}$ b) hold as $h_{j} \rightarrow 0$.

For any $\underline{\xi} \in\left[C_{0}^{\infty}(\Omega)\right]^{n}$, we deduce from 4.34 that $E_{\ell}^{r, h_{j}}\left(Q_{r}^{h_{j}}\right) \leqslant E_{\ell}^{r, h_{j}}\left(\underline{I}^{h_{j}} \xi\right)$. Passing to the $h_{j} \rightarrow 0$ limit in this, on noting (4.16), (4.9), 4.15b), (4.31), (4.28b) and (3.1), yields

$$
\left(\left|\underline{Q_{r}}\right|^{r}+\ell\left|\underline{\nabla} \cdot \underline{Q}_{r}-f\right|^{r}, 1\right)_{\Omega} \leqslant\left(|\underline{\xi}|^{r}+\ell|\underline{\nabla} \cdot \underline{\xi}-f|^{r}, 1\right)_{\Omega} \quad \forall \underline{\xi} \in\left[C_{0}^{\infty}(\Omega)\right]^{n} .
$$

Recalling that $\underline{V}_{0}^{r}(\Omega)$ is the strong closure of $\left[C_{0}^{\infty}(\Omega)\right]^{n}$ in the norm $\|\cdot\|_{\underline{V}^{r}(\Omega)}$ we conclude that (4.36 remains true for all $\underline{\xi} \in \underline{V}_{0}^{r}(\Omega)$, which is equivalent to $\left(\mathcal{U}_{r}\right)$, 3.34). As the solution of $\left(\mathcal{U}_{r}\right)$ is unique, the whole sequence $\left\{\underline{Q}_{r}^{h}\right\}_{h>0}$ converges in $4.28 \mathrm{a}$ b). Finally, the analogue of Remark 4.1 holds for $\left(\mathcal{U}_{r}^{h}\right)$. 
4.3 Approximation of $\left(\mathcal{P} \mathcal{M}_{r}\right)$

Similarly to Subsection 3.3 , we assume that the nonnegative $f^{(i)}, i=1 \rightarrow I$, for some $r_{0}>1$ are in $L^{r_{0}}(\Omega)$. In addition, for given constants $m \in\left(0, \min _{i=1 \rightarrow I}\left\{\int_{\Omega} f^{(i)}\right\}\right]$ and $G \geqslant m /\left|\Omega_{g}\right|$, we introduce the convex sets

$$
K_{G}^{h}(m):=\left\{\eta^{h} \in K_{G}^{h}: \int_{\Omega_{g}} \eta^{h} \mathrm{~d} \underline{x}=m\right\}, \quad \text { where } \quad K_{G}:=\left\{\eta^{h} \in S_{g}^{h}: 0 \leqslant \eta^{h} \leqslant G\right\} .
$$

For a given $r \in\left(1, r_{0}\right)$, we then consider the following fully practical approximation of $\left(\mathcal{P} \mathcal{M}_{r}\right)$ by $\underline{V}_{0}^{h}$ and $S^{h}$ :

$\left(\mathcal{P} \mathcal{M}_{r}^{h}\right)$ Find $\underline{Q}_{r}^{(i), h} \in \underline{V}_{0}^{h}, i=1 \rightarrow I$, and $g_{r}^{h} \in K_{G}^{h}(m)$ such that

$$
\begin{array}{r}
\left(\left|\underline{Q}_{r}^{(i), h}\right|^{r-2} \underline{Q}_{r}^{(i), h}, \underline{v}^{h}\right)^{h} \\
+\ell\left(\left|\underline{\nabla} \cdot \underline{Q}_{r}^{(i), h}-P^{h} f^{(i)}+g_{r}^{h}\right|^{r-2}\left(\underline{\nabla} \cdot \underline{Q}_{r}^{(i), h}-P^{h} f^{(i)}+g_{r}^{h}\right), \underline{\nabla} \cdot \underline{v}^{h}\right)_{\Omega}=0 \\
\forall \underline{v}^{h} \in \underline{V}_{0}^{h}, i=1 \rightarrow I, \\
\sum_{i=1}^{I}\left(\left|\underline{\nabla} \cdot \underline{Q}_{r}^{(i), h}-P^{h} f^{(i)}+g_{r}^{h}\right|^{r-2}\left(\underline{\nabla} \cdot \underline{Q}_{r}^{(i), h}-P^{h} f^{(i)}+g_{r}^{h}\right), \eta^{h}-g_{r}^{h}\right)_{\Omega_{g}} \geqslant 0 \\
\forall \eta^{h} \in K_{G}^{h}(m) .
\end{array}
$$

$\left(\mathcal{P} \mathcal{M}_{r}^{h}\right)$ is the Euler-Lagrange system for the following strictly convex minimization problem: Find $\underline{Q}_{r}^{(i), h} \in \underline{V}_{0}^{h}, i=1 \rightarrow I$, and $g_{r}^{h} \in K_{G}^{h}(m)$ such that

$$
\begin{aligned}
& E_{\ell}^{r, h}\left(\left\{\underline{Q}_{r}^{(i), h}\right\}_{i=1}^{I}, g_{r}^{h}\right) \leqslant E_{\ell}^{r, h}\left(\left\{\underline{v}^{(i), h}\right\}_{i=1}^{I}, \eta^{h}\right) \\
&:=\frac{1}{r} \sum_{i=1}^{I}\left[\left(\left|\underline{v}^{(i), h}\right|^{r}, 1\right)^{h}+\ell\left(\left|\underline{\nabla} \cdot \underline{v}^{(i), h}-P^{h} f^{(i)}+\eta^{h}\right|^{r}, 1\right)_{\Omega}\right] \\
& \forall \underline{v}^{(i), h} \in \underline{V}_{0}^{h}, i=1 \rightarrow I, \forall \eta^{h} \in K_{G}^{h}(m) .
\end{aligned}
$$

Hence, there exists a unique solution $\left(\left\{\underline{Q}_{r}^{(i), h}\right\}_{i=1}^{I}, g_{r}^{h}\right)$ to $\left(\mathcal{P} \mathcal{M}_{r}^{h}\right)$; and moreover, on noting 4.6) and 4.9, we have

$$
\sum_{i=1}^{I}\left[\left|\underline{Q}_{r}^{(i), h}\right|_{0, r, \Omega}^{r}+\ell\left|\underline{\nabla} \cdot \underline{Q}_{r}^{(i), h}-P^{h} f^{(i)}+g_{r}^{h}\right|_{0, r, \Omega}^{r}\right] \leqslant C_{\ell, r}\left[\frac{I m^{r}}{\left|\Omega_{g}\right|^{r-1}}+\sum_{i=1}^{I}\left|f^{(i)}\right|_{0, r, \Omega}^{r}\right] .
$$

It follows from 4.40 that there exist $\underline{Q}_{r}^{(i)} \in \underline{V}_{0}^{r}(\Omega), i=1 \rightarrow I$, and $g_{r} \in K_{G}(m)$ such that there exists a subsequence $\left\{\left\{\underline{Q}_{r}^{(i), h_{j}}\right\}_{i=1}^{I}, g_{r}^{h_{j}}\right\}_{h_{j}>0}$, where $\left(\left\{\underline{Q}_{r}^{(i), h}\right\}_{i=1}^{I}, g_{r}^{h}\right)$ is the unique solution of $\left(\mathcal{P} \mathcal{M}_{r}^{h}\right)$, such that

$$
\begin{aligned}
\underline{Q}_{r}^{(i), h_{j}} & \rightarrow \underline{Q}_{r}^{(i)} & \text { weakly in }\left[L^{r}(\Omega)\right]^{n}, & i=1 \rightarrow I, \\
\underline{\nabla} \cdot \underline{Q}_{r}^{(i), h_{j}} & \rightarrow \underline{\nabla} \cdot \underline{Q}_{r}^{(i)} & \text { weakly in } L^{r}(\Omega), & i=1 \rightarrow I, \\
g_{r}^{h_{j}} & \rightarrow g_{r} & \text { vaguely in } L^{\infty}\left(\Omega_{g}\right) &
\end{aligned}
$$

as $h_{j} \rightarrow 0$. 
For any $\xi^{(i)} \in\left[C_{0}^{\infty}(\Omega)\right]^{n}, i=1 \rightarrow I$, and $\eta \in K_{G}(m)$, we deduce from 4.39 that $E_{\ell}^{r, h_{j}}\left(\left\{Q_{r}^{(i), h_{j}}\right\}_{i=1}^{I}, g_{r}^{h_{j}}\right) \leqslant E_{\ell}^{r, h_{j}}\left(\left\{\underline{I}^{h_{j}} \xi^{(i)}\right\}_{i=1}^{I}, P^{h_{j}} \eta\right)$. Passing to the $h_{j} \rightarrow 0$ limit in this, and noting 4.16, 4.9, 4.15b, 4.41a-c) and (3.1), yields

$$
\begin{array}{r}
\sum_{i=1}^{I}\left(\left|\underline{Q}_{r}^{(i)}\right|^{r}+\ell \underline{\underline{\nabla}} \cdot \underline{Q}_{r}^{(i)}-f^{(i)}+\left.g_{r}\right|^{r}, 1\right)_{\Omega} \leqslant \sum_{i=1}^{I}\left(\left|\underline{\xi}^{(i)}\right|^{r}+\ell\left|\underline{\nabla} \cdot \underline{\xi}^{(i)}-f^{(i)}+\eta\right|^{r}, 1\right)_{\Omega} \\
\forall \underline{\xi}^{(i)} \in\left[C_{0}^{\infty}(\Omega)\right]^{n}, i=1 \rightarrow I, \forall \eta \in K_{G}(m) .
\end{array}
$$

Recalling that $\underline{V}_{0}^{r}(\Omega)$ is the strong closure of $\left[C_{0}^{\infty}(\Omega)\right]^{n}$ in the norm $\|\cdot\|_{\underline{V}^{r}(\Omega)}$ we see that 4.42 , remains true for all $\underline{\xi} \in \underline{V}_{0}^{r}(\Omega)$, which is equivalent to $\left.\left(\mathcal{P} \mathcal{M}_{r}\right), 3.46 \mathrm{a} b\right)$. As the solution of $\left(\mathcal{P} \mathcal{M}_{r}\right)$ is unique, the whole sequence $\left\{\left\{Q_{r}^{(i), h}\right\}_{i=1}^{I}, g_{r}^{h}\right\}_{h>0}$ converges in $\left.4.41 \mathrm{a}-\mathrm{c}\right)$. Finally, the analogue of Remark 4.1 holds for $\left(\mathcal{P} \mathcal{M}_{r}^{h}\right)$.

\section{Augmented Lagrangian methods for $\left(\mathcal{P}_{r}^{h}\right)$ and $\left(\mathcal{P} \mathcal{M}_{r}^{h}\right)$}

In this section we introduce, and prove convergence of, algorithms to solve the problems $\left(\mathcal{P}_{r}^{h}\right)$, 4.7a-e), and $\left.\left(\mathcal{P} \mathcal{M}_{r}^{h}\right), 4.38 \mathrm{a}, \mathrm{b}\right)$, which involve constraints, and hence Lagrange multipliers, as opposed to $\left(\mathcal{U}_{r}^{h}\right), 4.33$.

\section{$5.1\left(\mathcal{P}_{r}^{h}\right)$}

For a given constant $\mu>0$, we consider the following iterative method for solving the constrained problem $4.7 \mathrm{a}-\mathrm{e})$ :

For $k \geqslant 0$, given $u_{r}^{h, k} \in S^{h}$ and $\lambda_{r}^{ \pm, h, k} \in \mathbb{R}$, find $\underline{Q}_{r}^{h, k+1} \in \underline{V}_{0}^{h}$ and $q_{r}^{h, \pm, k+1} \in S_{ \pm}^{h}$ such that

$$
\begin{array}{r}
\left(\left|\underline{Q}_{r}^{h, k+1}\right|^{r-2} \underline{Q}_{r}^{h, k+1}, \underline{v}^{h}\right)^{h}+\mu\left(\underline{\nabla} \cdot \underline{Q}_{r}^{h, k+1}-\left[f_{r}^{+}-q_{r}^{+, h, k+1}\right]+\left[f_{r}^{-}-q_{r}^{-, h, k+1}\right], \underline{\nabla} \cdot \underline{v}^{h}\right)_{\Omega} \\
=\left(u_{r}^{h, k}, \underline{\nabla} \cdot \underline{v}^{h}\right)_{\Omega} \quad \forall \underline{v}^{h} \in \underline{V}_{0}^{h},
\end{array}
$$

$\ell\left(\left|q_{r}^{+, h, k+1}\right|^{r-2} q_{r}^{+, h, k+1}, v^{+, h}\right)_{\Omega^{+}}+\mu\left(\underline{\nabla} \cdot \underline{Q}_{r}^{h, k+1}-\left[f_{r}^{+}-q_{r}^{+, h, k+1}\right]+\left[f_{r}^{-}-q_{r}^{-, h, k+1}\right], v^{+, h}\right)_{\Omega^{+}}$

$$
\begin{array}{r}
-\mu\left[\left(f_{r}^{+}-q_{r}^{+, h, k+1}, 1\right)_{\Omega^{+}}-m\right]\left(v^{+, h}, 1\right)_{\Omega^{+}}=\left(u_{r}^{h, k}-\lambda_{r}^{+, h, k}, v^{+, h}\right)_{\Omega^{+}} \\
\forall v^{+, h} \in S_{+}^{h}, \quad(5.1 \mathrm{~b})
\end{array}
$$

$\ell\left(\left|q_{r}^{-, h, k+1}\right|^{r-2} q_{r}^{-, h, k+1}, v^{-, h}\right)_{\Omega^{-}}-\mu\left(\underline{\nabla} \cdot \underline{Q}_{r}^{h, k+1}-\left[f_{r}^{+}-q_{r}^{+, h, k+1}\right]+\left[f_{r}^{-}-q_{r}^{-, h, k+1}\right], v^{-, h}\right)_{\Omega^{-}}$

$-\mu\left[\left(f_{r}^{-}-q_{r}^{-, h, k+1}, 1\right)_{\Omega^{-}}-m\right]\left(v^{-, h}, 1\right)_{\Omega^{-}}=\left(-u_{r}^{h, k}-\lambda_{r}^{-, h, k}, v^{-, h}\right)_{\Omega^{-}} \quad \forall v^{-, h} \in S_{-}^{h}$.

Then for a given constant $\rho>0$, set

$$
\begin{aligned}
u_{r}^{h, k+1} & =u_{r}^{h, k}-\rho\left[\underline{\nabla} \cdot \underline{Q}_{r}^{h, k+1}-\left[P_{+}^{h} f_{r}^{+}-q_{r}^{+, h, k+1}\right]+\left[P_{-}^{h} f_{r}^{-}-q_{r}^{-, h, k+1}\right]\right] \in S^{h}, \\
\lambda_{r}^{ \pm, h, k+1} & =\lambda_{r}^{ \pm, h, k}-\rho\left[\left(f_{r}^{ \pm}-q_{r}^{ \pm, h, k+1}, 1\right)_{\Omega^{ \pm}}-m\right] \in \mathbb{R} .
\end{aligned}
$$

We introduce the augmented Lagrangian associated with the minimization problem, 4.12, which is equivalent to $\left(\mathcal{P}_{r}^{h}\right)$ : 


$$
\begin{aligned}
\mathcal{L}_{\ell, \mu}^{r, h}\left(\underline{v}^{h}, v^{+, h}, v^{-, h}, \eta^{h}, \lambda^{+}, \lambda^{-}\right) & \\
:= & \frac{1}{r}\left[\left(\left|\underline{v}^{h}\right|^{r}, 1\right)^{h}+\ell\left(\left|v^{+, h}\right|^{r}, 1\right)_{\Omega^{+}}+\ell\left(\left|v^{-, h}\right|^{r}, 1\right)_{\Omega^{-}}\right]-\lambda^{+}\left[\left(f_{r}^{+}-v^{+, h}, 1\right)_{\Omega^{+}}-m\right] \\
& -\lambda^{-}\left[\left(f_{r}^{-}-v^{-, h}, 1\right)_{\Omega^{-}}-m\right]-\left(\underline{\nabla} \cdot \underline{v}^{h}-\left[f_{r}^{+}-v^{+, h}\right]+\left[f_{r}^{-}-v^{-, h}\right], \eta^{h}\right)_{\Omega} \\
& +\frac{\mu}{2}\left[\left[\left(f_{r}^{+}-v^{+, h}, 1\right)_{\Omega^{+}}-m\right]^{2}+\left[\left(f_{r}^{-}-v^{-, h}, 1\right)_{\Omega^{-}}-m\right]^{2}\right] \\
+ & \frac{\mu}{2}\left|\underline{\nabla} \cdot \underline{v}^{h}-\left[f_{r}^{+}-v^{+, h}\right]+\left[f_{r}^{-}-v^{-, h}\right]\right|_{0, \Omega}^{2} \\
& \forall\left(\underline{v}^{h}, v^{+, h}, v^{-, h}, \eta^{h}, \lambda^{+}, \lambda^{-}\right) \in \underline{V}_{0}^{h} \times S_{+}^{h} \times S_{-}^{h} \times S^{h} \times \mathbb{R} \times \mathbb{R} .
\end{aligned}
$$

Then 5.1a-c) is the Euler-Lagrange system for the minimization of $\mathcal{L}_{\ell, \mu}^{r, h}\left(\underline{v}^{h}, v^{+, h}, v^{-, h}, u_{r}^{h, k}\right.$, $\lambda_{r}^{+, h, k}, \lambda_{r}^{-, h, k}$ ) over $\underline{v}^{h} \in \underline{V}_{0}^{h}$ and $v^{ \pm, h} \in S_{ \pm}^{h}$ for given $u_{r}^{h, k} \in S^{h}$ and $\lambda_{r}^{ \pm, h, k} \in \mathbb{R}$. As this is a strictly convex minimization problem, we have existence and uniqueness of $\underline{Q}_{r}^{h, k+1} \in \underline{V}_{0}^{h}$ and $q_{r}^{ \pm, h, k+1} \in S_{ \pm}^{h}$ solving $(5.1 \mathrm{a}-\mathrm{c})$.

THEOREM 5.1 For any $\mu>0$, and any $u_{r}^{h, 0} \in S^{h}$ and $\lambda_{r}^{ \pm, h, 0} \in \mathbb{R}$, if $\rho \in(0,2 \mu)$, then the sequence $\left\{\left(\underline{Q}_{r}^{h, k}, q_{r}^{+, h, k}, q_{r}^{-, h, k}, u_{r}^{h, k}, \lambda_{r}^{+, h, k}, \lambda_{r}^{-, h, k}\right)\right\}_{k} \geqslant 0$ generated by the algorithm $\left.5.1 \mathrm{a}-\mathrm{c}\right)$ and 5.2a, b) is such that

$$
\underline{Q}_{r}^{h, k} \rightarrow \underline{Q}_{r}^{h}, \quad q_{r}^{ \pm, h, k} \rightarrow q_{r}^{ \pm, h} \quad \text { as } k \rightarrow \infty,
$$

where $\left(Q_{r}^{h}, q_{r}^{ \pm, h}, u_{r}^{h}, \lambda_{r}^{ \pm, h}\right)$ is the unique solution of $\left.\left(\mathcal{P}_{r}^{h}\right), 4.7 \mathrm{a}-\mathrm{e}\right)$.

Proof. Let $\bar{Q}^{k}:=Q_{r}^{h}-Q_{r}^{h, k}, \bar{q}^{ \pm, k}:=q_{r}^{ \pm, h}-q_{r}^{ \pm, h, k}, \bar{u}^{k}:=u_{r}^{h}-u_{r}^{h, k}$ and $\bar{\lambda}^{ \pm, k}:=\lambda_{r}^{ \pm, h}-\lambda_{r}^{ \pm, h, k}$. Then from $(4.7 \mathrm{a}-\mathrm{e})$ and $(5.1 \mathrm{a}-\mathrm{c})$ we have

$$
\begin{aligned}
\left(\left|\underline{Q}_{r}^{h}\right|^{r-2} \underline{Q}_{r}^{h}-\left|\underline{Q}_{r}^{h, k+1}\right|^{r-2} \underline{Q}_{r}^{h, k+1}, \bar{Q}^{k+1}\right)^{h}+\mu\left|\underline{\nabla} \cdot \bar{Q}^{k+1}+\bar{q}^{+, k+1}-\bar{q}^{-, k+1}\right|_{0, \Omega}^{2} \\
\quad+\ell\left(\left|q_{r}^{+, h}\right|^{r-2} q_{r}^{+, h}-\left|q_{r}^{+, h, k+1}\right|^{r-2} q_{r}^{+, h, k+1}, \bar{q}^{+, k+1}\right)_{\Omega^{+}}+\mu\left[\left(\bar{q}^{+, k+1}, 1\right)_{\Omega^{+}}\right]^{2} \\
\quad+\ell\left(\left|q_{r}^{-, h}\right|^{r-2} q_{r}^{-, h}-\left|q_{r}^{-, h, k+1}\right|^{r-2} q_{r}^{-, h, k+1}, \bar{q}^{-, k+1}\right)_{\Omega^{-}}+\mu\left[\left(\bar{q}^{-, k+1}, 1\right)_{\Omega^{-}}\right]^{2} \\
=\left(\bar{u}^{k}, \underline{\nabla} \cdot \underline{\bar{Q}}^{k+1}+\bar{q}^{+, k+1}-\bar{q}^{-, k+1}\right)_{\Omega}-\left(\bar{\lambda}^{+, k}, \bar{q}^{+, k+1}\right)_{\Omega^{+}}-\left(\bar{\lambda}^{-, k}, \bar{q}^{-, k+1}\right)_{\Omega^{-}} ;
\end{aligned}
$$

and from $4.7 \mathrm{~d} / \mathrm{e})$ and $(5.2 \mathrm{a} \mathrm{b})$

$$
\begin{aligned}
\left|\bar{u}^{k+1}\right|_{0, \Omega}^{2}=\left|\bar{u}^{k}\right|_{0, \Omega}^{2}-2 \rho\left(\bar{u}^{k}, \underline{\nabla} \cdot \underline{\bar{Q}}^{k+1}+\bar{q}^{+, k+1}-\bar{q}^{-, k+1}\right)_{\Omega} & \\
& +\rho^{2}\left|\underline{\nabla} \cdot \underline{\bar{Q}}^{k+1}+\bar{q}^{+, k+1}-\bar{q}^{-, k+1}\right|_{0, \Omega}^{2}, \\
\left|\bar{\lambda}^{ \pm, k+1}\right|^{2}=\left|\bar{\lambda}^{ \pm, k}\right|^{2}+2 \rho \bar{\lambda}^{ \pm, k}\left(\bar{q}^{ \pm, k+1}, 1\right)_{\Omega^{ \pm}} & +\rho^{2}\left[\left(\bar{q}^{ \pm, k+1}, 1\right)_{\Omega^{ \pm}}\right]^{2} .
\end{aligned}
$$

Combining (5.5) and 5.6a, b) yields

$$
\begin{aligned}
& \left|\bar{u}^{k+1}\right|_{0, \Omega}^{2}+\left|\bar{\lambda}^{+, k+1}\right|^{2}+\left|\bar{\lambda}^{-, k+1}\right|^{2}+2 \rho\left(\left|\underline{Q}_{r}^{h}\right|^{r-2} \underline{Q}_{r}^{h}-\left|\underline{Q}_{r}^{h, k+1}\right|^{r-2} \underline{Q}_{r}^{h, k+1}, \underline{Q}^{k+1}\right)^{h} \\
& \quad+2 \rho \ell\left(\left|q_{r}^{+, h}\right|^{r-2} q_{r}^{+, h}-\left|q_{r}^{+, h, k+1}\right|^{r-2} q_{r}^{+, h, k+1}, \bar{q}^{+, k+1}\right)_{\Omega^{+}} \\
& \quad+2 \rho \ell\left(\left|q_{r}^{-, h}\right|^{r-2} q_{r}^{-, h}-\left|q_{r}^{-, h, k+1}\right|^{r-2} q_{r}^{-, h, k+1}, \bar{q}^{-, k+1}\right)_{\Omega^{-}} \\
& +\left(2 \rho \mu-\rho^{2}\right)\left[\left|\underline{\nabla} \cdot \underline{\bar{Q}}^{k+1}+\bar{q}^{+, k+1}-\bar{q}^{-, k+1}\right|_{0, \Omega}^{2}+\left[\left(\bar{q}^{+, k+1}, 1\right)_{\Omega^{+}}\right]^{2}+\left[\left(\bar{q}^{-, k+1}, 1\right)_{\Omega^{-}}\right]^{2}\right] \\
& \quad=\left|\bar{u}^{k}\right|_{0, \Omega}^{2}+\left|\bar{\lambda}^{+, k}\right|^{2}+\left|\bar{\lambda}^{-, k}\right|^{2} .
\end{aligned}
$$


Hence for $\rho \in(0,2 \mu)$, the sequence $\left\{\left|\bar{u}^{k}\right|_{0, \Omega}^{2}+\left|\bar{\lambda}^{+, k}\right|^{2}+\left|\bar{\lambda}^{-, k}\right|^{2}\right\}_{k} \geqslant 1$ is monotonically decreasing and bounded below. Therefore it must converge to a limit, and hence the desired result (5.4) holds on noting 3.2 .

\section{$5.2\left(\mathcal{P} \mathcal{M}_{r}^{h}\right)$}

For given constants $\mu, \rho>0$, we consider the following iterative method for solving the constrained problem $4.38 \mathrm{a}, \mathrm{b})$ :

For $k \geqslant 0$, given $F_{0, r}^{h, k}, F_{G, r}^{h, k} \in S_{g}^{h}$ and $\lambda_{r}^{h, k} \in \mathbb{R}$, find $\underline{Q}_{r}^{(i), h, k+1} \in \underline{V}_{0}^{h}, i=1 \rightarrow I$, and $g_{r}^{h, k+1} \in S_{g}^{h}$ such that

$$
\begin{aligned}
& \left(\left|\underline{Q}_{r}^{(i), h, k+1}\right|^{r-2} \underline{Q}_{r}^{(i), h, k+1}, \underline{v}^{h}\right)^{h} \\
& +\ell\left(\left|\underline{\nabla} \cdot \underline{Q}_{r}^{(i), h, k+1}-P^{h} f^{(i)}+g_{r}^{h, k+1}\right|^{r-2}\left(\underline{\nabla} \cdot \underline{Q}_{r}^{(i), h, k+1}-P^{h} f^{(i)}+g_{r}^{h, k+1}\right), \underline{\nabla} \cdot \underline{v}^{h}\right)_{\Omega}=0 \\
& \forall \underline{v}^{h} \in \underline{V}_{0}^{h}, i=1 \rightarrow I, \\
& \ell \sum_{i=1}^{I}\left(\left|\underline{\nabla} \cdot \underline{Q}_{r}^{(i), h, k+1}-P^{h} f^{(i)}+g_{r}^{h, k+1}\right|^{r-2}\left(\underline{\nabla} \cdot \underline{Q}_{r}^{(i), h, k+1}-P^{h} f^{(i)}+g_{r}^{h, k+1}\right), \eta^{h}\right) \Omega_{g} \\
& -\left(\left[F_{0, r}^{h, k}-2 \rho g_{r}^{h, k+1}\right]_{+}-\left[F_{G, r}^{h, k}+2 \rho\left(g_{r}^{h, k+1}-G\right)\right]_{+}, \eta^{h}\right) \Omega_{g} \\
& +\mu\left[\left(g_{r}^{h, k+1}, 1\right)_{\Omega_{g}}-m\right]\left(\eta^{h}, 1\right)_{\Omega_{g}}=-\left(\lambda_{r}^{h, k}, \eta^{h}\right)_{\Omega_{g}} \quad \forall \eta^{h} \in S_{g}^{h}
\end{aligned}
$$

Then set

$$
\begin{aligned}
& F_{0, r}^{h, k+1}=\left[F_{0, r}^{h, k}-2 \rho g_{r}^{h, k+1}\right]_{+} \in S_{g}^{h}, \quad F_{G, r}^{h, k+1}=\left[F_{G, r}^{h, k}+2 \rho\left(g_{r}^{h, k+1}-G\right)\right]_{+} \in S_{g}^{h}, \\
& \lambda_{r}^{h, k+1}=\lambda_{r}^{h, k}+\rho\left[\left(g_{r}^{h, k+1}, 1\right)_{\Omega_{g}}-m\right] \in \mathbb{R} .
\end{aligned}
$$

We introduce an augmented Lagrangian associated with the minimization problem 4.39):

$$
\begin{aligned}
& \mathcal{L}_{\ell, \mu, \rho}^{r, h}\left(\left\{\underline{v}^{(i), h}\right\}_{i=1}^{I}, \eta^{h}, F_{0}^{h}, F_{G}^{h}, \lambda\right) \\
&:= \frac{1}{r} \sum_{i=1}^{I}\left[\left(\left|\underline{v}^{(i), h}\right|^{r}, 1\right)^{h}+\ell\left(\left|\underline{\nabla} \cdot \underline{v}^{(i), h}-P^{h} f^{(i)}+\eta^{h}\right|^{r}, 1\right)_{\Omega}\right]+\lambda\left[\left(\eta^{h}, 1\right)_{\Omega_{g}}-m\right] \\
&+\frac{\mu}{2}\left[\left(\eta^{h}, 1\right)_{\Omega_{g}}-m\right]^{2}+\frac{1}{4 \rho}\left(\left[F_{0}^{h}-2 \rho \eta^{h}\right]_{+}^{2}+\left[F_{G}^{h}+2 \rho\left(\eta^{h}-G\right)\right]_{+}^{2}, 1\right)_{\Omega_{g}} \\
& \quad \forall \underline{v}^{(i), h} \in \underline{V}_{0}^{h}, i=1 \rightarrow I, \forall \eta^{h}, F_{0}^{h}, F_{G}^{h} \in S_{g}^{h}, \forall \lambda \in \mathbb{R} .
\end{aligned}
$$

Here $F_{0}^{h}, F_{G}^{h} \in S_{g}^{h}$ are Lagrange multipliers associated with the inequality constraints on the convex set $K_{G}$. Then $5.8 \mathrm{a}$ b) is the Euler-Lagrange system for the minimization of $\mathcal{L}_{\ell, \mu, \rho}^{r, h}\left(\left\{\underline{v}^{(i), h}\right\}_{i=1}^{I}, \eta^{h}\right.$, $\left.F_{0, r}^{h, k}, F_{G, r}^{h, k}, \lambda_{r}^{h, k}\right)$ over $\underline{v}^{(i), h} \in \underline{V}_{0}^{h}, i=1 \rightarrow I$, and $\eta^{h} \in S_{g}^{h}$ for given $F_{0, r}^{h, k}, F_{G, r}^{h, k} \in S_{g}^{h}$ and $\lambda_{r}^{h, k} \in \mathbb{R}$. As this is a strictly convex minimization problem, we have existence and uniqueness of $\underline{Q}_{r}^{(i), h, k+1} \in \underline{V}_{0}^{h}, i=1 \rightarrow I$, and $g_{r}^{h, k+1} \in S_{g}^{h}$ solving 5.8a b).

THEOREM 5.2 For any $\mu>0$, and any $F_{0, r}^{h, 0}, F_{G, r}^{h, 0} \in S_{g}^{h}$ and $\lambda_{r}^{h, 0} \in \mathbb{R}$, if $\rho \in(0,2 \mu)$, then the sequence $\left\{\left(\left\{\underline{Q}_{r}^{(i), h, k}\right\}_{i=1}^{I}, g_{r}^{h, k}, F_{0, r}^{h, k}, F_{G, r}^{h, k}, \lambda_{r}^{h, k}\right)\right\}_{k \geqslant 0}$ generated by the algorithm 5.8ab) and 
(5.9a, b) is such that

$$
\underline{Q}_{r}^{(i), h, k} \rightarrow \underline{Q}_{r}^{(i), h}, \quad i=1 \rightarrow I, \quad g_{r}^{h, k} \rightarrow g_{r}^{h} \quad \text { as } k \rightarrow \infty,
$$

where $\left(\left\{\underline{Q}_{r}^{(i), h}\right\}_{i=1}^{I}, g_{r}^{h}\right)$ is the unique solution of $\left.\left(\mathcal{P} \mathcal{M}_{r}^{h}\right), 4.38 \mathrm{a} b\right)$.

Proof. Firstly, we note that the unique solution $\left(\left\{\underline{Q}_{r}^{(i), h}\right\}_{i=1}^{I}, g_{r}^{h}\right)$ of $\left(\mathcal{P} \mathcal{M}_{r}^{h}\right), 4.38 \mathrm{a}$ b) solves 4.38a and

$$
\begin{aligned}
& \ell \sum_{i=1}^{I}\left(\left|\underline{\nabla} \cdot \underline{Q}_{r}^{(i), h}-P^{h} f^{(i)}+g_{r}^{h}\right|^{r-2}\left(\underline{\nabla} \cdot \underline{Q}_{r}^{(i), h}-P^{h} f^{(i)}+g_{r}^{h}\right), \eta^{h}\right)_{\Omega_{g}} \\
&=\left(F_{0, r}^{h}-F_{G, r}^{h}-\lambda_{r}^{h}, \eta^{h}\right)_{\Omega_{g}} \quad \forall \eta^{h} \in S_{g}^{h}
\end{aligned}
$$

for some $\lambda_{r}^{h} \in \mathbb{R}$, and where

$$
F_{0, r}^{h}=\left[F_{0, r}^{h}-2 \rho g_{r}^{h}\right]_{+} \in S_{g}^{h}, \quad F_{G, r}^{h}=\left[F_{G, r}^{h}+2 \rho\left(g_{r}^{h}-G\right)\right]_{+} \in S_{g}^{h} .
$$

Let $Z_{r}^{(i), h}:=\underline{\nabla} \cdot \underline{Q}_{r}^{(i), h}-P^{h} f^{(i)}+g_{r}^{h} \in S^{h}$ and $Z_{r}^{(i), h, k}:=\underline{\nabla} \cdot \underline{Q}_{r}^{(i), h, k}-P^{h} f^{(i)}+g_{r}^{h, k} \in S^{h}$. In addition, let $\bar{Q}^{(i), k}:=\underline{Q}_{r}^{(i), h}-\underline{Q}_{r}^{(i), h, k}, \bar{Z}^{(i), k}:=Z_{r}^{(i), h}-Z_{r}^{(i), h, k}, \bar{g}^{k}:=g_{r}^{h}-g_{r}^{h, k}, \bar{F}_{0}^{k}:=$ $F_{0, r}^{h}-F_{0, r}^{h, k}, \bar{F}_{G}^{k}:=F_{G, r}^{h}-F_{G, r}^{h, k}$ and $\bar{\lambda}^{k}:=\lambda_{r}^{h}-\lambda_{r}^{h, k}$. Then from 4.38a, 5.12 and 5.8ab) we have

$$
\begin{aligned}
& \sum_{i=1}^{I}\left(\left|\underline{Q}_{r}^{(i), h}\right|^{r-2} \underline{Q}_{r}^{(i), h}-\left|\underline{Q}_{r}^{(i), h, k+1}\right|^{r-2} \underline{Q}_{r}^{(i), h, k+1}, \underline{\bar{Q}}^{(i), k+1}\right)^{h} \\
& \quad+\ell \sum_{i=1}^{I}\left(\left|Z_{r}^{(i), h}\right|^{r-2} Z_{r}^{(i), h}-\left|Z_{r}^{(i), h, k+1}\right|^{r-2} Z_{r}^{(i), h, k+1}, \bar{Z}^{k}\right)_{\Omega}+\mu\left[\left(\bar{g}^{k+1}, 1\right)_{\Omega_{g}}\right]^{2} \\
& =\left(\bar{F}_{0}^{k+1}-\bar{F}_{G}^{k+1}-\bar{\lambda}^{k}, \bar{g}^{k+1}\right)_{\Omega_{g}} ;
\end{aligned}
$$

and from 5.13 and 5.9ab) we have

$$
\begin{aligned}
\left|\bar{F}_{0}^{k+1}\right|_{0, \Omega_{g}}^{2} & \leqslant\left(\bar{F}_{0}^{k+1}, \bar{F}_{0}^{k}-2 \rho \bar{g}^{k+1}\right)_{\Omega_{g}}, \quad\left|\bar{F}_{G}^{k+1}\right|_{0, \Omega_{g}}^{2} \leqslant\left(\bar{F}_{G}^{k+1}, \bar{F}_{G}^{k}+2 \rho \bar{g}^{k+1}\right)_{\Omega_{g}}, \\
\left|\bar{\lambda}^{k+1}\right|^{2} & =\left|\bar{\lambda}^{k}\right|^{2}+2 \rho \bar{\lambda}^{k}\left(\bar{g}^{k+1}, 1\right)_{\Omega_{g}}+\rho^{2}\left[\left(\bar{g}^{k+1}, 1\right)_{\Omega_{g}}\right]^{2} .
\end{aligned}
$$

Combining (5.14) and 5.15a,b) yields

$$
\begin{aligned}
\left|\bar{F}_{0}^{k+1}\right|_{0, \Omega_{g}}^{2} & +\left|\bar{F}_{G}^{k+1}\right|_{0, \Omega_{g}}^{2}+\left|\bar{\lambda}^{k+1}\right|^{2}+\left(2 \rho \mu-\rho^{2}\right)\left[\left(\bar{g}^{k+1}, 1\right)_{\Omega_{g}}\right]^{2} \\
& +2 \rho \sum_{i=1}^{I}\left(\left|\underline{Q}_{r}^{(i), h}\right|^{r-2} \underline{Q}_{r}^{(i), h}-\left|\underline{Q}_{r}^{(i), h, k+1}\right|^{r-2} \underline{Q}_{r}^{(i), h, k+1}, \underline{Q}^{(i), k+1}\right)^{h} \\
& +2 \rho \ell \sum_{i=1}^{I}\left(\left|Z_{r}^{(i), h}\right|^{r-2} Z_{r}^{(i), h}-\left|Z_{r}^{(i), h, k+1}\right|^{r-2} Z_{r}^{(i), h, k+1}, \bar{Z}^{k+1}\right)_{\Omega} \\
& \leqslant\left|\bar{\lambda}^{k}\right|^{2}+\left(\bar{F}_{0}^{k+1}, \bar{F}_{0}^{k}\right)_{\Omega_{g}}+\left(\bar{F}_{G}^{k+1}, \bar{F}_{G}^{k}\right)_{\Omega_{g}} \\
& \leqslant\left|\bar{\lambda}^{k}\right|^{2}+\frac{1}{2}\left[\left|\bar{F}_{0}^{k+1}\right|_{0, \Omega_{g}}^{2}+\left|\bar{F}_{G}^{k+1}\right|_{0, \Omega_{g}}^{2}+\left|\bar{F}_{0}^{k}\right|_{0, \Omega_{g}}^{2}+\left|\bar{F}_{G}^{k}\right|_{0, \Omega_{g}}^{2}\right] .
\end{aligned}
$$


Hence for $\rho \in(0,2 \mu)$, the sequence $\left\{\left|\bar{F}_{0}^{k}\right|_{0, \Omega_{g}}^{2}+\left|\bar{F}_{G}^{k}\right|_{0, \Omega_{g}}^{2}+2\left|\bar{\lambda}^{k}\right|^{2}\right\}_{k \geqslant 1}$ is monotonically decreasing and bounded below. Therefore it must converge to a limit, and hence the desired result 5.11) holds on noting (3.2).

\section{Solution of the nonlinear algebraic systems}

All the resulting nonlinear algebraic systems obtained above for $\left(\mathcal{P}_{r}^{h}\right),\left(\mathcal{U}_{r}^{h}\right)$ and $\left(\mathcal{P} \mathcal{M}_{r}^{h}\right)$ contain the same type of nonlinearity, $|v|^{r-2} v$ or its vector form, and this is solved iteratively. This is in addition to the external iterative loops, described in Section 5 above, to satisfy the constraints in the case of the partial MK, $\left(\mathcal{P}_{r}^{h}\right)$, and optimal matching $\left(\mathcal{P} \mathcal{M}_{r}^{h}\right)$, problems. Clearly, replacing this nonlinear term by $\left|v^{j}\right|^{r-2} v^{j+1}$ at the $(j+1)^{\text {th }}$ iteration is not possible as $r<2$ and $\left|v^{j}\right|$ can be zero. Our iterative scheme is based instead on the following regularized representation of this nonlinear term:

$$
\left|v^{j}\right|_{\varepsilon}^{r-2}\left(v^{j+1 / 2}-v^{j}\right)+\left|v^{j}\right|^{r-2} v^{j},
$$

where $|v|_{\varepsilon}:=\sqrt{|v|^{2}+\varepsilon^{2}}$ for $0<\varepsilon \ll 1$, and so all terms are well defined. In addition, overrelaxation $v^{j+1}=\alpha v^{j+1 / 2}+(1-\alpha) v^{j}$ with $\alpha \geqslant 1$ is used to accelerate the convergence.

Below we present such an iterative procedure for the solution of the nonlinear system, $(5.1 \mathrm{a}-\mathrm{c})$, arising at the $(k+1)^{\text {th }}$ iteration of the augmented Lagrangian method applied to $\left(\mathcal{P}_{r}^{h}\right)$. Given approximations to the Lagrange multipliers $u_{r}^{h, k} \in S^{h}$ and $\lambda_{r}^{ \pm, h, k} \in \mathbb{R}$, and on setting $Q_{r}^{h, k+1,0}=$ $\underline{Q}_{r}^{h, k} \in \underline{V}_{0}^{h}$ and $\underline{q}_{r}^{ \pm, h, k+1,0}=\underline{q}_{r}^{ \pm, h, k} \in S_{ \pm}^{h}$, we apply the following iterative loop for $j \geqslant 0$, where to simplify the notation, we will omit the indices involving $k, r$ and $h$ on the iterates:

Given $\underline{Q}^{j} \equiv \underline{Q}_{r}^{h, k+1, j} \in \underline{V}_{0}^{h}$ and $q^{ \pm, j} \equiv q_{r}^{ \pm, h, k+1, j} \in S_{ \pm}^{h}$, find $\underline{Q}^{j+1 / 2} \in \underline{V}_{0}^{h}$ and $q^{ \pm, j+1 / 2} \in$ $S_{ \pm}^{h}$ such that

$$
\begin{aligned}
&\left(\left|\underline{Q}^{j}\right|_{\varepsilon}^{r-2} \underline{Q}^{j+1 / 2}, \underline{v}^{h}\right)^{h}+\mu\left(\underline{\nabla} \cdot \underline{Q}^{j+1 / 2}+q^{+, j+1 / 2}-q^{j+1 / 2}, \underline{\nabla} \cdot \underline{v}^{h}\right)_{\Omega} \\
&=\left(u+\mu f^{+}-\mu f^{-}, \underline{\nabla} \cdot \underline{v}^{h}\right)_{\Omega}+\left(\left[\left|\underline{Q}^{j}\right|_{\varepsilon}^{r-2}-\left|\underline{Q}^{j}\right|^{r-2}\right] \underline{Q}^{j}, \underline{v}^{h}\right)^{h} \quad \forall \underline{v}^{h} \in \underline{V}_{0}^{h}, \\
& \ell\left(\left|q^{+, j}\right|_{\varepsilon}^{r-2} q^{+, j+1 / 2}, v^{+, h}\right)_{\Omega^{+}}+\mu\left(\underline{\nabla} \cdot \underline{Q}^{j+1 / 2}+q^{+, j+1 / 2}-q^{-, j+1 / 2}, v^{+, h}\right)_{\Omega^{+}} \\
&=\left(u+\mu f^{+}-\mu f^{-}-\lambda^{+}, v^{+, h}\right)_{\Omega^{+}}+\ell\left(\left[\left|q^{+, j}\right|_{\varepsilon}^{r-2}-\left|q^{+, j}\right|^{r-2}\right] q^{+, j}, v^{+, h}\right)_{\Omega^{+}} \\
& \quad+\mu\left[\left(f^{+}-q^{+, j}, 1\right)_{\Omega^{+}}-m\right]\left(v^{h,+}, 1\right)_{\Omega^{+}} \quad \forall v^{h,+} \in S_{+}^{h}, \\
& \ell\left(\left|q^{-, j}\right|_{\varepsilon}^{r-2} q^{-, j+1 / 2}, v^{-, h}\right)_{\Omega^{-}}-\mu\left(\underline{\nabla} \cdot \underline{Q}^{j+1 / 2}+q^{+, j+1 / 2}-q^{-, j+1 / 2}, v^{-, h}\right)_{\Omega^{-}} \\
&=\left(-u-\mu f^{+}+\mu f^{-}-\lambda^{-}, v^{-, h}\right)_{\Omega^{-}}+\ell\left(\left[\left|q^{-, j}\right|_{\varepsilon}^{r-2}-\left|q^{-, j}\right|^{r-2}\right] q^{-, j}, v^{-, h}\right)_{\Omega^{-}} \\
& \quad+\mu\left[\left(f^{-}-q^{-, j}, 1\right)_{\Omega^{-}}-m\right]\left(v^{-, h}, 1\right)_{\Omega^{-}} \quad \forall v^{-, h} \in S_{-}^{h} .
\end{aligned}
$$

Then set $Q^{j+1}=\alpha Q^{j+1 / 2}+(1-\alpha) Q^{j}$ and $q^{ \pm, j+1}=\alpha q^{ \pm, j+1 / 2}+(1-\alpha) q^{ \pm, j}$, and repeat the above iteration until the difference of iterates satisfies a given tolerance before updating the Lagrange multipliers. Note that the terms $\left[\left(f^{ \pm}-q^{ \pm, j}, 1\right)_{\Omega^{ \pm}}-m\right]$ are lagged in order to maintain the sparsity of the linear system $6.2 \mathrm{a}-\mathrm{c})$.

The unbalanced problem $\left(\mathcal{U}_{r}^{h}\right)$ is unconstrained, and was solved using an iterative scheme similar to $6.2 \mathrm{a}-\mathrm{c})$, without any exterior Lagrange multiplier loop.

The additional nonlinearity, $\xi(t):=[t]_{+}$, had to be dealt with in the partial matching problem, $\left(\mathcal{P} \mathcal{M}_{r}^{h}\right)$, because the augmented Lagrangian algorithm was employed also for the inequality 
constraints $g_{r}^{h} \geqslant 0$ and $g_{r}^{h} \leqslant G$. This nondifferentiable nonlinearity was regularized as $\xi_{\varepsilon}(t):=$ $\left|[t]_{+}\right|_{\varepsilon}-\varepsilon \equiv \sqrt{[t]_{+}^{2}+\varepsilon^{2}}-\varepsilon$ for $0<\varepsilon \ll 1$, and linearized at each iteration, similarly to 6.1 .

\section{Numerical experiments}

To test our numerical schemes we solved several two-dimensional examples. The Matlab Partial Differential Equations toolbox was used for the domain triangulation, and subdomains, $\Omega^{ \pm}$and $\Omega_{g}$, with curved boundaries were approximated by a union of triangles. We refer to [4] for the Matlab realization of the lowest order Raviart-Thomas element in $\mathbb{R}^{2}$. With probability one any delta functions used as sources or sinks lied in the interior of a triangle, so no smoothing of these discrete sources/sinks was necessary.

Solutions to MK and optimal matching problems are often singular; so to approximate such solutions and to determine more accurately the free boundaries of the transportation domains we used an adaptive finite element mesh; see [5] for the details of the refinement/coarsening algorithm. In the partial MK problems, $\left(\mathcal{P}_{r}^{h}\right)$, the mesh was refined wherever the fluxes $\underline{Q}_{r}^{h}$ and $q_{r}^{ \pm, h}$ changed rapidly. Typically, these rapid changes of the auxiliary fluxes $q_{r}^{ \pm, h}$ occur on the free boundaries of transportation domains; we used this observation to determine the free boundaries efficiently. Although the auxiliary fluxes are eliminated from the unbalanced and optimal matching problems, $\left(\mathcal{U}_{r}^{h}\right)$ and $\left(\mathcal{P} \mathcal{M}_{r}^{h}\right)$, we calculated them when solving these problems for the purposes of mesh adaptation and the determination of the free boundary. Coarse initial meshes with 13001800 triangles have been adaptively refined twice in most examples below, yielding final meshes containing several thousand triangles.

In all the examples below we chose $r=1+10^{-7}$ and $\varepsilon=10^{-7}$. The domain $\Omega$ was either a unit square, in which case we chose $\ell=1 / \sqrt{2}$, or a unit square with a section removed; in the latter case the value $\ell=2$ was sufficient to satisfy the necessary condition 2.1 . We note that, provided (2.1) was satisfied, the value of $\ell$ did not influence the solution and the auxiliary fluxes were always nonnegative. However, the value of $\ell$ did influence the convergence of the iterations and, although for the smaller value of $\ell$ we used over-relaxation with $\alpha=1.5$, which led to a moderate acceleration in the partial and unbalanced MK problems, computations with $\ell=2$ were performed with $\alpha=1$ (no over-relaxation). Similarly, no over-relaxation was used for the partial optimal matching problems.

The parameters for the augmented Lagrangian method for the equality and the inequality constraints in all examples were $\mu=\rho=1$. The iterations have been performed until all constraints were satisfied up to a given tolerance $\varepsilon_{A L}$. Thus, for the partial MK problems, the conditions were

$$
\begin{gathered}
m^{-1}\left|\underline{\nabla} \cdot \underline{Q}_{r}^{h, k}-\left[P_{+}^{h} f_{r}^{+}-q_{r}^{+, h, k}\right]+\left[P_{-}^{h} f_{r}^{-}-q_{r}^{-, h, k}\right]\right|_{0,1, \Omega} \leqslant \varepsilon_{A L}, \\
m^{-1}\left|\left(f_{r}^{ \pm}-q_{r}^{ \pm, h, k}, 1\right)_{\Omega^{ \pm}}-m\right| \leqslant \varepsilon_{A L} ;
\end{gathered}
$$

and, for the partial matching problems,

$$
m^{-1}\left|\left(g_{r}^{h, k}, 1\right)_{\Omega_{g}}-m\right| \leqslant \varepsilon_{A L}, \quad-\varepsilon_{A L} \leqslant g_{r}^{h, k} \leqslant G\left(1+\varepsilon_{A L}\right) .
$$

Given a tolerance $\varepsilon_{N L}$, on the $k^{\text {th }}$ iteration of the augmented Lagrangian algorithm the solution of the nonlinear algebraic system $(5.1 \mathrm{a}-\mathrm{c})$ was computed using $(6.2 \mathrm{a}-\mathrm{c})$ until the tolerance $\varepsilon_{N L}^{k}=$ $\varepsilon_{N L} / \sqrt{k}$ was achieved, so that the accuracy increased gradually with $k$. More precisely, let $\mathcal{E}^{h}$ be 
the set of all internal edges and $\underline{\phi}_{e}(\underline{x})$ the basis vector function associated with the edge $e \in \mathcal{E}^{h}$ in the Raviart-Thomas finite element space $\underline{V}_{0}^{h}$ (see [4]). Then any $\underline{v}^{h} \in \underline{V}_{0}^{h}$ can be represented as $\sum_{e \in \mathcal{E}^{h}} v_{e}^{h} \underline{\phi}_{e}$ and we define the norm $\left\|\underline{v}^{h}\right\|_{\mathcal{E}^{h}}:=\sum_{e \in \mathcal{E}^{h}}|e|\left|v_{e}^{h}\right|$. Our stopping criterion was

$$
\frac{\left\|\underline{Q}^{j+1}-\underline{Q}^{j}\right\|_{\mathcal{E}^{h}}}{\left\|\underline{Q}^{j+1}\right\|_{\mathcal{E}^{h}}} \leqslant \varepsilon_{N L}^{k} \quad \text { and } \quad \frac{\left|q^{ \pm, j+1}-q^{ \pm, j}\right|_{0,1, \Omega^{ \pm}}}{\left|q_{r}^{ \pm, j+1}\right|_{0,1, \Omega^{ \pm}}} \leqslant \varepsilon_{N L}^{k}
$$

in the partial MK problems, $6.2 \mathrm{a}-\mathrm{c}$ ); only the first quantity, with $\varepsilon_{N L}^{k}=\varepsilon_{N L}$, in 7.3 for the unbalanced problems, and

$$
\max _{i=1 \rightarrow I}\left\{\frac{\left\|\underline{Q}^{(i), j+1}-\underline{Q}^{(i), j}\right\|_{\mathcal{E}^{h}}}{\left\|\underline{Q}^{(i), j+1}\right\|_{\mathcal{E}^{h}}}\right\} \leqslant \varepsilon_{N L}^{k} \quad \text { and } \quad \frac{\left|g^{j+1}-g^{j}\right|_{0,1, \Omega_{g}}}{\left|g^{j+1}\right|_{0,1, \Omega_{g}}} \leqslant \varepsilon_{N L}^{k}
$$

for the partial matching problems. It should be noted that it was better to update the multipliers after a few iterations of the nonlinear loop without necessarily satisfying the stopping criteria $(7.3)$ and (7.4); and demand the fulfilment of these conditions only near the convergence of the augmented Lagrangian algorithm. Finally, we noticed that in all the partial matching examples that we solved, the optimal distributions $g_{r}^{h}$ were nonnegative even if the constraint $g_{r}^{h} \geqslant 0$ was omitted, and not taken into account in the numerical procedure. We do not know, however, whether this condition is automatically satisfied for all optimal partial matching problems.

The computation times for the examples below were approximately one hour for the partial MK problems, and 15-20 minutes for the unbalanced MK and partial matching problems; all computations have been performed on a personal computer.

EXAMPLE 1 (Partial MK problem, two ellipses) Let $f^{+}=1$ and $f^{-}=2$ inside their supports $\Omega^{ \pm}$, the left and right ellipses, respectively (Fig. 2, top left), with semiaxes $a^{+}=2 a^{-}=$ $0.2, b^{+}=b^{-}=0.45$, and the distance $d=0.6$ between their axes of vertical symmetry. We chose $m=\frac{1}{2} \int_{\bar{\Omega}} f^{+}=\frac{1}{2} \int_{\bar{\Omega}} f^{-}=\pi a^{-} b^{-}$. In this case it is cheapest to move the mass along horizontal paths from the right half of the left ellipse to the left half of the right one, with the axes of vertical symmetry of the ellipses as the exact free boundaries. Elementary integration gives the total cost of such transportation: $\mathcal{C}=a^{-} b^{-}\left(\pi d-4 a^{-}\right)=0.06682$. The auxiliary fluxes $q^{ \pm}$are zero inside the regions from/to where the mass is transported and are equal to $f^{ \pm}$outside. Numerical calculations reproduced well this behavior and, since these jumps occur at the free boundaries, in our numerical simulations these boundaries are shown as the level contours $q_{r}^{ \pm, h}=\frac{1}{2} \max q_{r}^{ \pm, h}$. The approximate total cost was calculated on an adapted mesh of about nine thousand triangles as $\int_{\bar{\Omega}}|\underline{Q}| \approx \sum_{\sigma \in \mathcal{T}^{h}}|\sigma|\left|\underline{Q}_{r}^{h}\left(\underline{o}_{\sigma}\right)\right|=0.06671$, where $\underline{o}_{\sigma}$ denotes the centre of triangle $\sigma$. In this example $\varepsilon_{A L}=\varepsilon_{N L}=10^{-4}$.

EXAMPLE 2 (Partial MK problem, two ellipses and an obstacle) Similar to the previous example but with $\Omega$ being the unit square with a section removed, which leads to a change in the transport paths (see Fig. 2, top right). The transport density becomes singular near the tip of the obstacle, with the approximate total cost being 0.07776 . The exact solution is unknown; the numerical results were obtained with $\varepsilon_{A L}=2 \cdot 10^{-5}, \varepsilon_{N L}=10^{-4}$ and a mesh containing about five thousand triangles (Fig. 2, bottom).

EXAMPLE 3 (Partial MK problem with discrete sinks) Let $f^{+}$be constant inside its support $\Omega^{+}$, the circle with radius $R_{0}=0.4$, centre $(0.5,0.5)$, and with total mass $\int_{\bar{\Omega}} f^{+}=1$; let $f^{-}$be the 


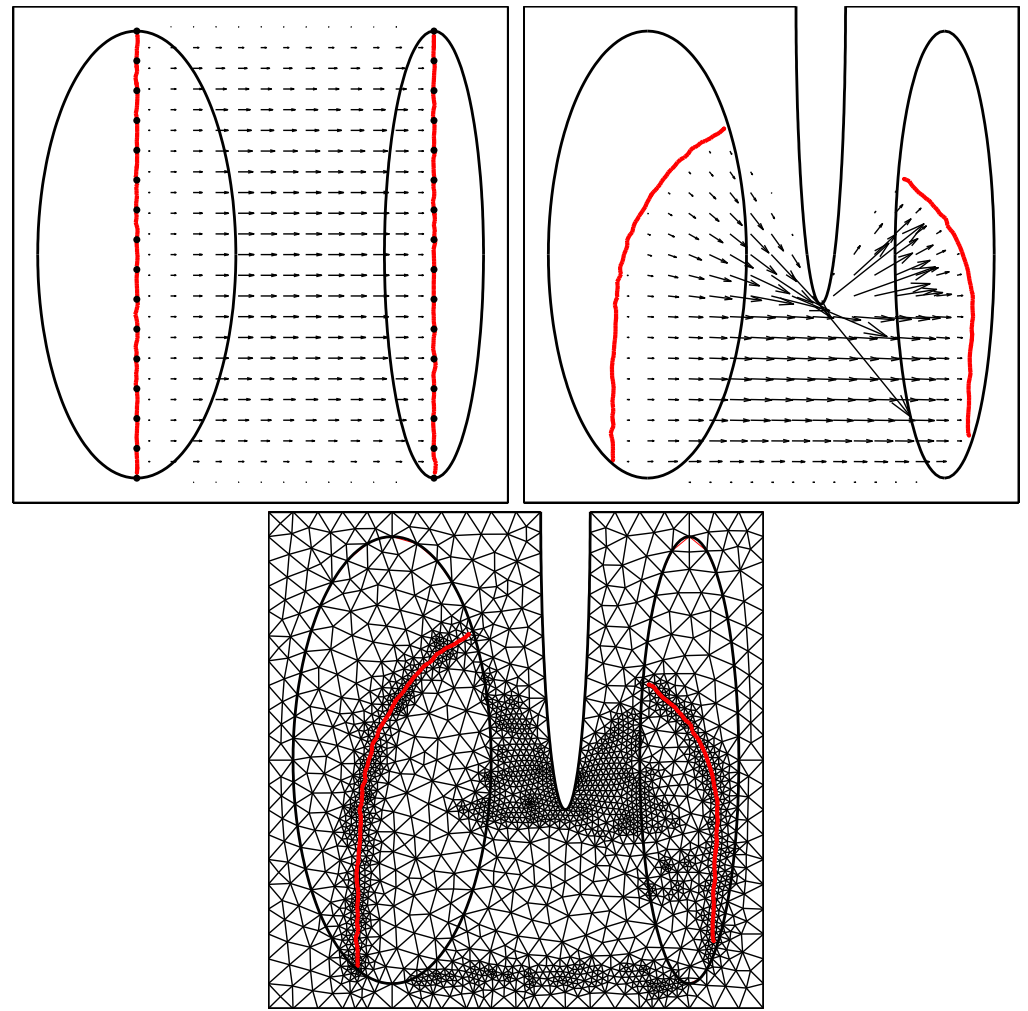

FIG. 2. Partial MK problem. The left and right ellipses are supports of constant $f^{+}$and $f^{-}$, respectively, and $m=$ $\frac{1}{2} \int \bar{\Omega} f^{+}=\frac{1}{2} \int \bar{\Omega} f^{-}$. Top left: $\Omega$ is a unit square; top right: same with a section removed leading to an obstacle to transportation; bottom: the adapted mesh. Shown: the computed flux (arrows); the computed free boundary (red lines; for colours, see pdf file); black dots in the left plot indicate the known position of the exact free boundaries.

sum of three unit delta functions placed at $(0.1,0.8),(0.4,0.5)$ and $(0.7,0.7)$ (Fig. 3). We chose $m=1 / 2$. It is easy to see that mass from each point of the transportation domain should in this case be transported to the closest $\delta$-sink (each of them remains unsaturated) and all points on the boundary of this transportation domain must be at the same unknown distance $r_{0}$ from the closest sink. Taking into account all possible intersections of the main circle and three circles of radius $r$ centred at the sinks, one can calculate the integral of $f^{+}$over such a domain as a function $s(r)$. Solving the nonlinear algebraic equation $s\left(r_{0}\right)=m$, we found that $r_{0}=0.2034$ and were able to plot the exact free boundary together with the boundary determined numerically, which is represented by the level contour $q_{r}^{+, h}=\frac{1}{2} \max q_{r}^{+, h}$. The approximate masses transported to each sink were, respectively, $0.0461,0.2484$ and 0.2055 . In this example $\varepsilon_{A L}=0.5 \cdot 10^{-4}, \varepsilon_{N L}=10^{-4}$ and, to clarify the solution structure, we plotted not the optimal flux itself but the directions of optimal transportation, the vector field $\underline{Q}_{r}^{h} /\left|\underline{Q}_{r}^{h}\right|_{\varepsilon}$.

EXAMPLE 4 (Unbalanced MK problem, two ellipses) The same as Examples 1 and 2, except $f^{-}=1$ inside the right ellipse, so $m=\frac{1}{2} \int_{\bar{\Omega}} f^{+}=\int_{\bar{\Omega}} f^{-}$and so the problems are unbalanced. In 

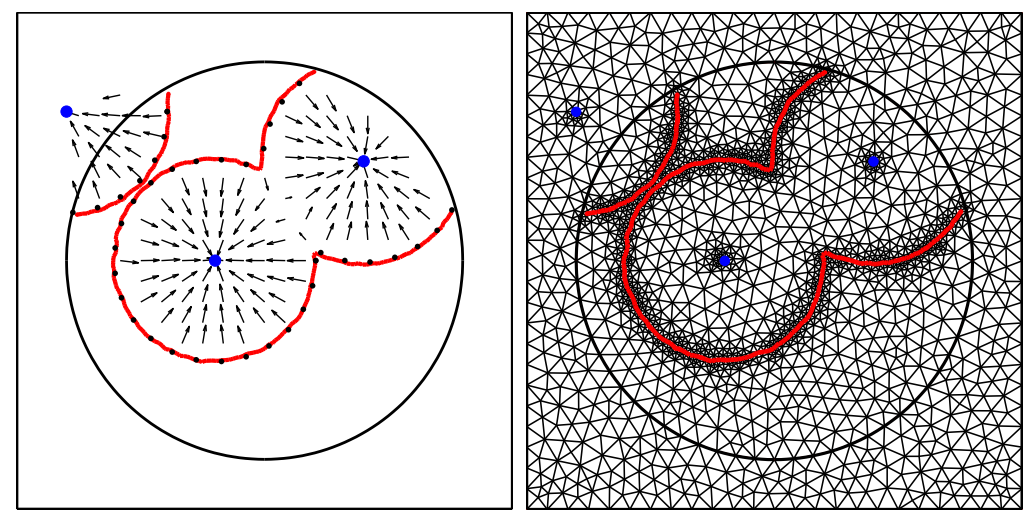

FIG. 3. Partial MK problem. $f^{+}$is distributed uniformly in the circle and has the total mass $\int_{\bar{\Omega}} f^{+}=1 ; f^{-}$is the sum of three point sinks each of mass one (the blue dots). Left: the optimal transportation plan for $m=1 / 2$; right: the adapted mesh, about four thousand triangles. Shown: the computed directions of optimal transportation (arrows); the computed free boundary (red lines); black dots in the left plot indicate the position of the exact boundary.

the first case (Fig. 4. left) $\Omega$ is the unit square and the optimal plan is again to move the mass from the right half of the left ellipse along the horizontal paths; the only free boundary is the vertical axis of symmetry of this ellipse, and the auxiliary flux $q^{+}=f^{+}-\underline{\nabla}$. $\underline{Q}$ has a jump on this boundary. The total cost of transportation, found by means of elementary integration, is $\mathcal{C}=a^{-} b^{-}\left(\pi \mathrm{d}-\frac{8}{3} a^{-}\right)=$ 0.07282. The numerical solution of $\left(\mathcal{U}_{r}^{h}\right)$ in this example was obtained on a crude mesh adapted only once and containing about three thousand triangles with $\varepsilon_{N L}=4 \cdot 10^{-6}$. Nevertheless, we obtained an accurate total cost estimate, $\sum_{\sigma \in \mathcal{T}^{h}}|\sigma|\left|\underline{Q}_{r}^{h}\left(\underline{o}_{\sigma}\right)\right|=0.07270$. The free boundary (red line), approximated by the level contour $q_{r}^{+, h}=\frac{1}{2} \max q_{r}^{+, h}$, is also close to the exact boundary (black dots). The problem with an obstacle (Fig. 4, right) was solved on a mesh with about eight thousand triangles, with $\varepsilon_{N L}=5 \cdot 10^{-5}$, yielding an approximate cost 0.08971 .
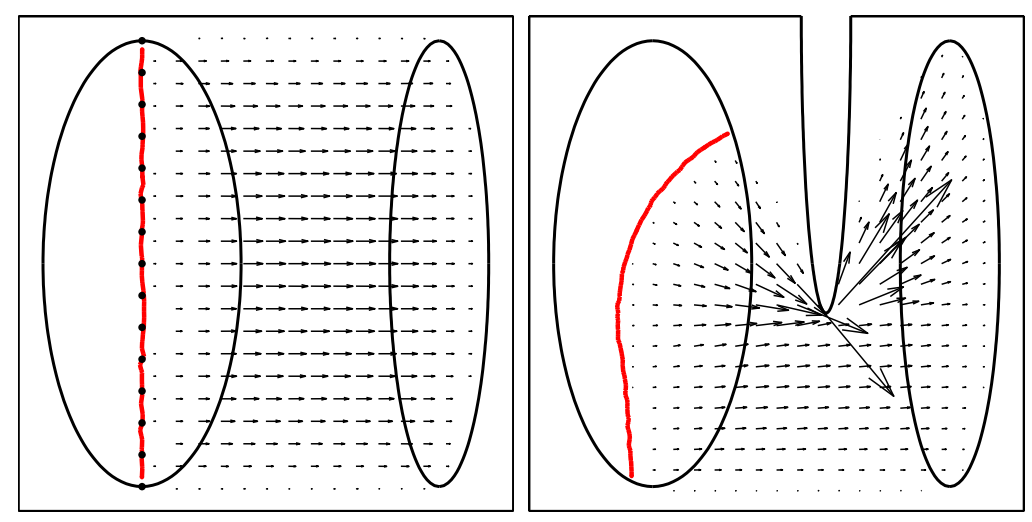

FIG. 4. Unbalanced MK problem. As in Fig. 2 but with $m=\frac{1}{2} \int_{\bar{\Omega}} f^{+}=\int_{\bar{\Omega}} f^{-}$. 

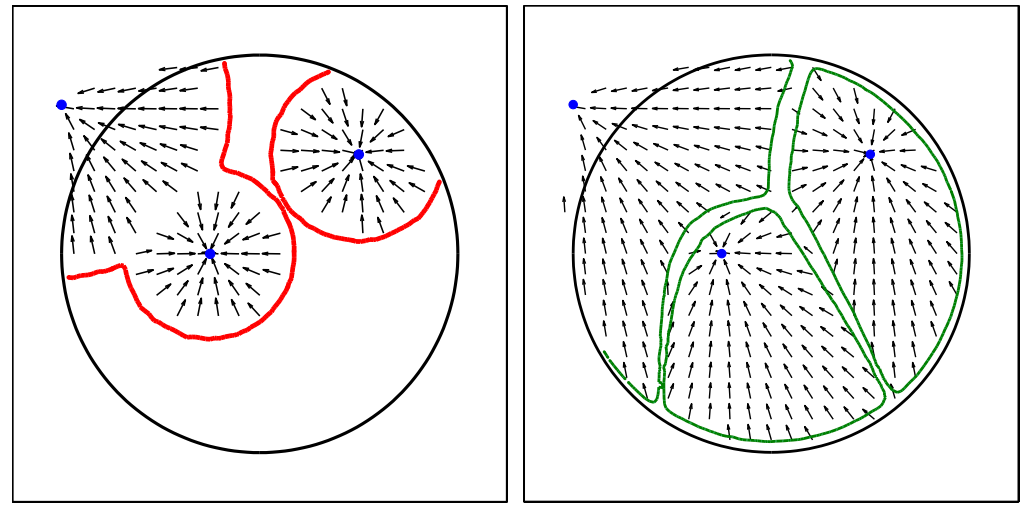

FIG. 5. Unbalanced and balanced MK problem with three discrete sources; as in Fig. 3 except the sink weights. Left: each sink is a delta function with weight $\frac{1}{6}$, hence $m=\int_{\bar{\Omega}} f^{-}=\frac{1}{2} \int_{\bar{\Omega}} f^{+}$. Right: each sink has weight $\frac{1}{3}$ and hence the problem is balanced, $m=\int_{\bar{\Omega}} f^{-}=\int_{\bar{\Omega}} f^{+}$. The green lines are the approximate boundaries of the sink attraction domains.

EXAMPLE 5 (Unbalanced and balanced MK problems with discrete sinks) If, in the configuration of Example 3, we take $f^{-}$to be the sum of three delta functions at the same locations as before, but now each with its weight equal to the mass transported to that sink in Example 3, then the problem is an unbalanced one with the same solution. Solving the unbalanced problem, $\left(\mathcal{U}_{r}^{h}\right)$, on a mesh of approximately five thousand triangles with $\varepsilon_{N L}=3 \cdot 10^{-5}$ we obtained free boundaries that are visually identical to those obtained in Example 3; and the total cost estimates differed by $0.6 \%$. We present two more examples with the same source and three equal point sinks in the same locations as before. In the first one (Fig. 5, left) the delta sinks each have the weight $1 / 6$; this is again an unbalanced problem with $m=1 / 2$. In the second the weights are $1 / 3$, so $m=\int \bar{\Omega} f^{+}=\int_{\bar{\Omega}} f^{-}$and the problem is balanced; and hence there is no free boundary as both auxiliary fluxes are zero. The solution (Fig. 5, right) was obtained from $\left(\mathcal{U}_{r}^{h}\right)$ with about thirteen thousand triangles, and $\varepsilon_{N L}=10^{-5}$. Here the support of $f^{+}$is divided into three attraction domains corresponding to the three sinks. On the boundaries of the attraction domains $Q$ is zero and, approximately, these boundaries are represented by the level contour $\left|\underline{Q}_{r}^{h}\right|=2 \cdot 10^{-3} \max \left|\underline{Q}_{r}^{h}\right|$ (green line). We note that in the balanced MK problem $\underline{\nabla} \cdot \underline{Q}=f^{+}-f^{-}$and, numerically, we obtained $\left|P^{h}\left(f^{+}-f^{-}\right)-\underline{\nabla} \cdot \underline{Q}_{r}^{h}\right|_{0, \infty, \Omega}=2 \cdot 10^{-6}$.

EXAMPLE 6 (Partial matching problem, two ellipses) The ellipses in Fig. 6 are as in Example 1. We set $f^{(1)}=1$ in the left ellipse, $f^{(2)}=2$ in the right one; the matching mass $m=\frac{1}{2} \int_{\bar{\Omega}} f^{(1)}=\frac{1}{2} \int_{\bar{\Omega}} f^{(2)}=0.045 \pi$, and $\Omega_{g}$ is the rectangle between the two ellipses having width 0.2 . We chose first $G=1$ (Fig. 6, left). Since $G\left|\Omega_{g}\right|>m$, a solution to $(\mathcal{P} \mathcal{M}$ ) exists. In this case, the transport to $\Omega_{g}$ along horizontal paths from the right half of the left ellipse and the left half of the right one is admissible; this is the cheapest matching transportation. Note, however, that for each pair of the meeting transport rays the matching with any distribution of $g$ upon their common segment lying in $\bar{\Omega}_{g}$ has the same total cost, so there are infinitely many optimal matching plans satisfying $g \in[0, G]$. However, a selection is made by our regularized formulation, $\left(\mathcal{P} \mathcal{M}_{r}\right)$. The total cost of optimal matching is equal to the transportation cost in Example 1, $\mathcal{C}=0.06682$. Solving $\left(\mathcal{P} \mathcal{M}_{r}^{h}\right)$ using an adapted mesh with about eight thousand triangles and $\varepsilon_{A L}=10^{-4}$, $\varepsilon_{N L}=10^{-3}$, we obtained $\mathcal{C} \approx \sum_{\sigma \in \mathcal{T} h}|\sigma|\left[\left|\underline{Q}_{r}^{(1), h}\left(\underline{o}_{\sigma}\right)\right|+\left|\underline{Q}_{r}^{(2), h}\left(\underline{o}_{\sigma}\right)\right|\right]=0.06671$. 

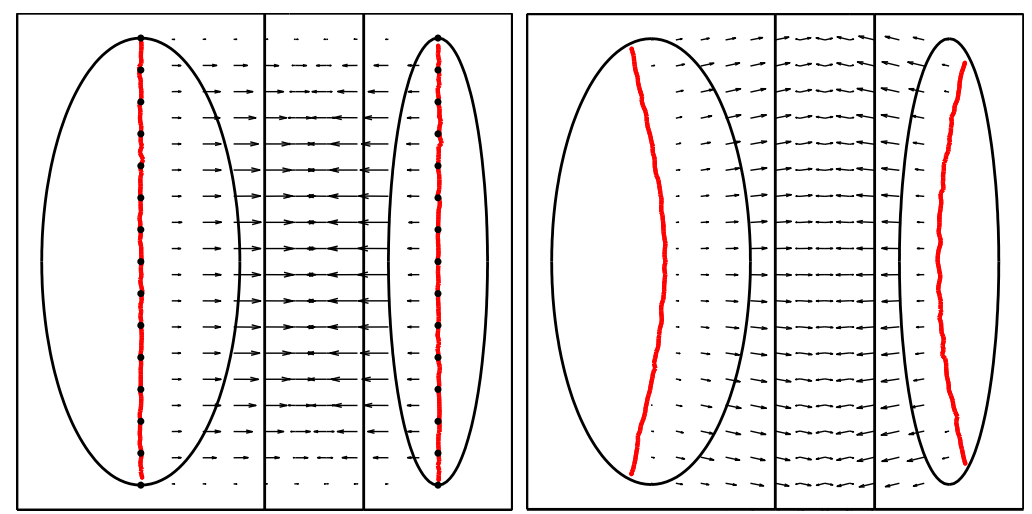

FIG. 6. Partial matching problems. Left: $G=1$, right: $G=m /\left|\Omega_{g}\right|$. Shown: the boundaries of the domain $\Omega_{g}$ and the supports of $f^{(1)}$ and $f^{(2)}$ (black lines); the optimal fluxes $\underline{Q}_{r}^{(1), h}$ and $Q_{r}^{(2), h}$ (arrows); the computed boundaries of the transportation domains (red lines). The exact boundaries are indicated by the black dots (left).

For $G=m /\left|\Omega_{g}\right|$ (Fig. 6, right) the optimal matching plan also exists; in this case $g$ must be equal to $G$ in the whole set $\bar{\Omega}_{g}$ and transportation along only horizontal rays is now not possible. A numerical solution was obtained on a mesh with about seven thousand triangles, $\varepsilon_{A L}=\varepsilon_{N L}=10^{-4}$, and the estimated cost was $\mathcal{C} \approx 0.06821$.

EXAMPLE 7 (Partial matching problem, two rectangles) Let $f^{(1)}=f^{(2)}=1$ in their supports, the left and right rectangles, respectively; and the circle be the matching domain $\Omega_{g}$ (see Fig. 77). We set $m=\frac{1}{3} \int_{\bar{\Omega}} f^{(1)}=\frac{1}{2} \int_{\bar{\Omega}} f^{(2)}$. Solving $\left(\mathcal{P} \mathcal{M}_{r}^{h}\right)$ with $G=2 m /\left|\Omega_{g}\right|$ (Fig. 7 left), we obtain, within the chosen tolerances, $g_{r}^{h}=G$ in half of $\Omega_{g}$ and $g_{r}^{h}=0$ in the other half; in this case the mesh contained about five thousand triangles. The matching domain shrinks as the value of
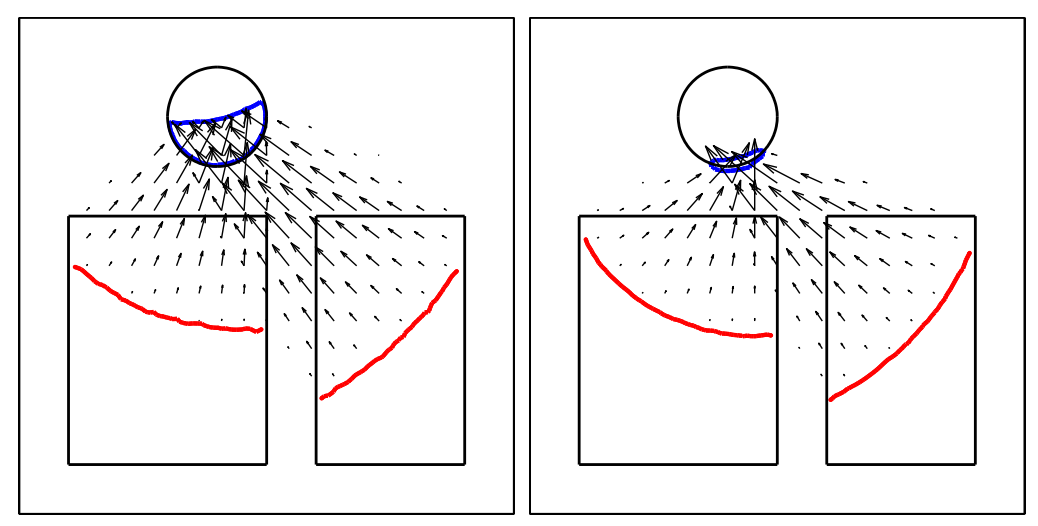

FIG. 7. Partial matching problems. Left: $G=2 m /\left|\Omega_{g}\right|$, right: $G \approx \infty$. Shown: the boundaries of the domain $\Omega g$ and the supports of $f^{(1)}$ and $f^{(2)}$ (black lines); the optimal fluxes $\underline{Q}_{r}^{(1), h}$ and $\underline{Q}_{r}^{(2), h}$ (arrows); the computed boundaries of the transportation domains (red lines) and of the matching domains (blue lines). 
$G$ increases; in the limit $G \rightarrow \infty$ (not covered by our theoretical analysis) the optimal matching occurs at the leading edge of the boundary of $\Omega_{\mathrm{g}}$. Solving the same problem with $G=10^{10}$ and an adapted mesh of about 3500 triangles (Fig. 7 , right), we found that $g_{r}^{h}$ is supported in a single layer of triangles along the leading border of $\Omega_{g}$; the constraint $g \leqslant G$ was inactive in this case. In both of these examples we set $\varepsilon_{A L}=10^{-4}$ and $\varepsilon_{N L}=10^{-3}$.

\section{Acknowledgements}

We acknowledge support of the Sixth EU Framework Programme-Transnational Access implemented as Specific Support Action (Dryland Research SSA).

\section{REFERENCES}

1. Ambrosio, L. Lecture notes on optimal transport. In: L. Ambrosio et al., Mathematical Aspects of Evolving Interfaces, Lecture Notes in Math. 1812, Springer (2003), 1-52. ZZbl 1047.35001 MR 2011032

2. Ambrosio, L., Fusco, N., \& Pallara, D. Functions of Bounded Variation and Free Discontinuity Problems. Clarendon Press, Oxford (2000). Zbl 0957.49001 MR 1857292

3. Angenent, S., Haker, S., \& Tannenbaum, A. Minimizing flows for the Monge-Kantorovich problem. SIAM J. Math. Anal. 35 (2003), 61-97. Zbl 1042.49040 MR 2001465

4. Bahriawati, C., \& Carstensen, C. Three Matlab implementations of the lowest-order RaviartThomas MFEM with a posteriori error control. Comput. Methods Appl. Math. 5 (2005), 333-361. Zbl 1086.65107 MR 2194203

5. Barrett, J. W., \& Prigozhin, L. A mixed formulation of the Monge-Kantorovich equations. Math. Model. Numer. Anal. 41 (2007), 1041-1066. Zbl 1132.35333 MR 2377106

6. Benamou, J.-D. Numerical resolution of an unbalanced mass transport problem. Math. Model. Numer. Anal. 37 (2003), 851-868. Zbl 1037.65063 MR 2020867

7. Benamou, J.-D., \& BREnier, Y. A computational fluid mechanics solution to the Monge-Kantorovich mass transfer problem. Numer. Math. 84 (2000), 375-393. Zbl 0968.76069 MR 1738163

8. Bouchitté, G., Buttazzo, G., \& Seppecher, P. Shape optimization solutions via MongeKantorovich equation. C. R. Acad. Sci. Paris 324 (1997), 1185-1191. Zbl $0884.49023 \mid$ MR 1451945

9. Buttazzo, G., \& Stepanov, E. Transport density in Monge-Kantorovich problems with Dirichlet conditions. Discrete Contin. Dynam. Systems 12 (2005), 607-628. Zbl 1094.49028 MR 2127841

10. Caffarelli, L. A., \& McCann, R. J. Free boundaries in optimal transport and Monge-Ampère obstacle problems. Ann. of Math. (to appear).

11. EKeland, I. An optimal matching problem. ESAIM Control Optim. Calc. Var. 11 (2005), 57-71. Zbl 1106.49054 MR 2110613

12. Evans, L. C. Weak Convergence Methods for Nonlinear Partial Differential Equations. CBMS Reg. Conf. Ser. Math. 74, Amer. Math. Soc., Providence, RI (1990). Zbl 0698.35004 MR 1034481

13. Evans, L. C. Partial differential equations and Monge-Kantorovich mass transfer. Current Developments in Mathematics, Int. Press, Boston, MA (1997), 65-126. Zbl 0954.35011 MR 1698853

14. Evans, L. C., \& Gangbo, W. Differential equations methods for the Monge-Kantorovich mass transfer problem. Mem. Amer. Math. Soc. 137 (1999), no. 653. Zbl 0920.49004 MR 1464149

15. Farhloul, M. A mixed finite element method for a nonlinear Dirichlet problem. IMA J. Numer. Anal. 18 (1998), 121-132. Zbl 0909.65086 MR 1492051

16. Feldman, M., \& MCCANN, R. J. Uniqueness and transport density in Monge's mass transportation problem. Calc. Var. Partial Differential Equations 15 (2002), 81-113. Zbl 1003.49031 MR 1920716

17. Folland, G. B. Real Analysis: Modern Techniques and their Applications. 2nd ed., Wiley-Interscience, New York (1984). Zbl 0549.28001 MR 0767633 
18. Girault, V., \& RaViart, P.-A. Finite Element Methods for Navier-Stokes Equations. Springer (1986). Zbl 0585.65077 MR 0851383

19. Pratelli, A. Equivalence between some definitions for the optimal mass transport problem and for transport density on manifolds. Ann. Mat. Pura Appl. 184 (2005), 215-238. Zbl 1099.49030 MR 2149093

20. Roberts, J. E., \& Thomas, J.-M. Mixed and hybrid methods. Handbook of Numerical Analysis, Vol. II, P. G. Ciarlet and J.-L. Lions (eds.), North-Holland, Amsterdam (1991), 523-639. Z Zbl 0875.65090 MR 1115239

21. VILlani, C. Topics in Optimal Transportation. Grad. Stud. Math. 58, Amer. Math. Soc., Providence, RI (2003). Zbl 1106.90001 MR 1964483 